\title{
Genetic interaction of Per- and Dec-genes in the mammalian circadian clock
}

\author{
Dissertation \\ for the award of the degree \\ "Doctor rerum naturalium" \\ Division of Mathematics and Natural Sciences \\ of the Georg-August-Universität Göttingen
}

submitted by

Brid Bode

from Wolfenbüttel

Göttingen 2011 
Thesis Committee:

Dr. Henrik Oster, Circadian Rhythms Group, Max Planck Institute for Biophysical Chemistry (Reviewer)

Prof. Dr. Julia Fischer, Cognitive Ethology, German Primate Center (Reviewer)

Prof. Dr. Walter Paulus, Dept. of Clinical Neurophysiology, Georg-August-University Göttingen

Date of the oral examination: 31.05 .2011 
Brid Bode

Gartenstraße 3

37073 Göttingen

Göttingen, 20.04.2011

Affidavit that the thesis with the title 'genetic interaction of Per- and Dec-genes in the mammalian circadian clock' submitted by me, Brid Bode, was written independently and with no other sources and aids than quoted.

Brid Bode 
I Summary

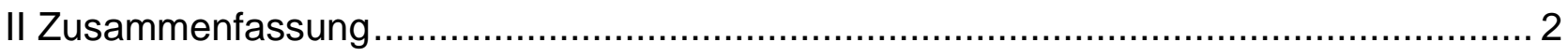

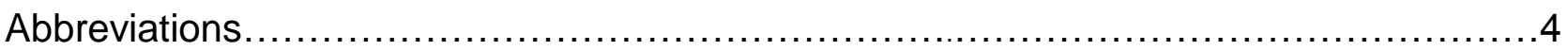

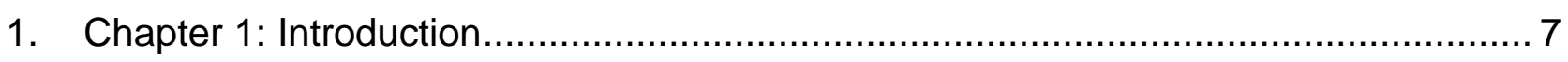

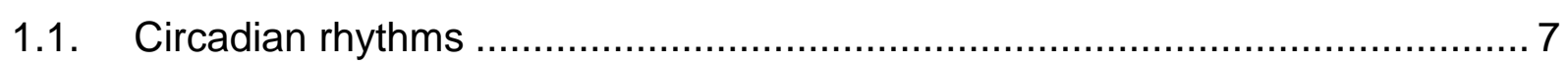

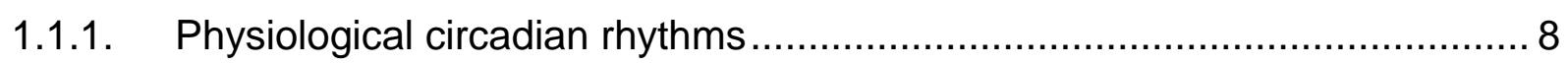

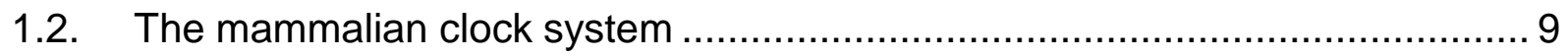

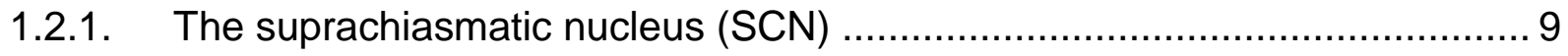

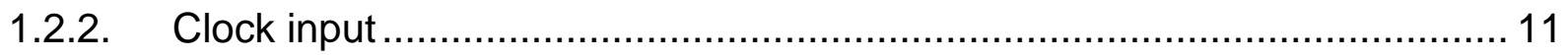

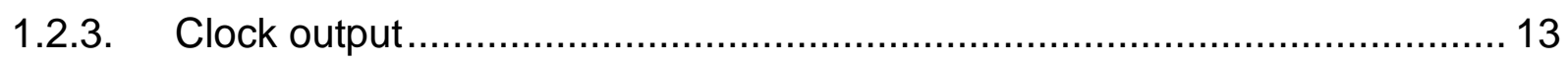

1.2.4. The molecular clockwork ............................................................... 14

1.2.4.1. Transcriptional/translational feedback loops ......................................... 14

1.2.4.2. Dec1 and Dec2 ........................................................................ 17

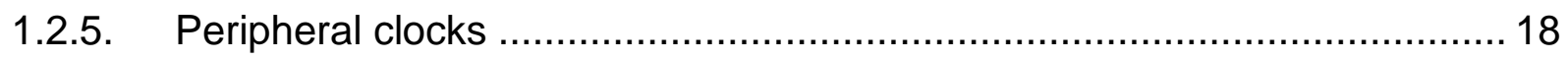

1.3. Clock mutants.................................................................................... 19

1.4. Photic resetting of the circadian system .............................................. 21

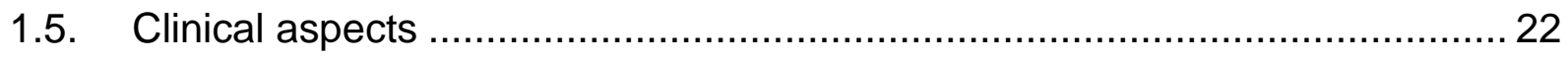

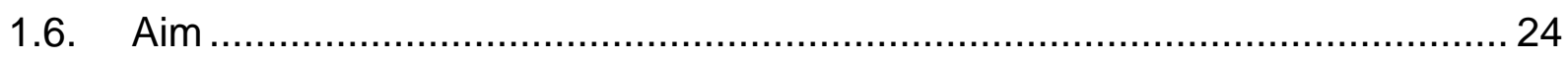

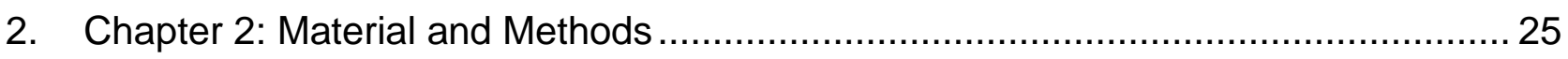

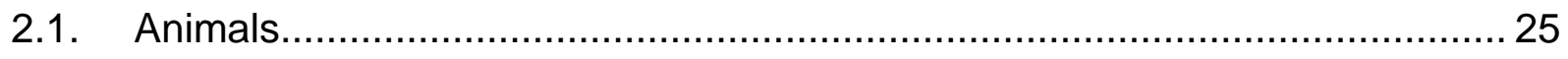

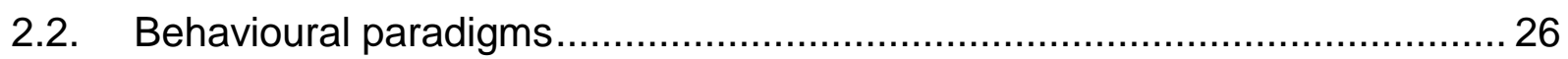

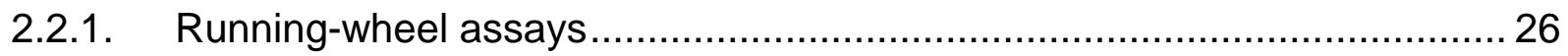

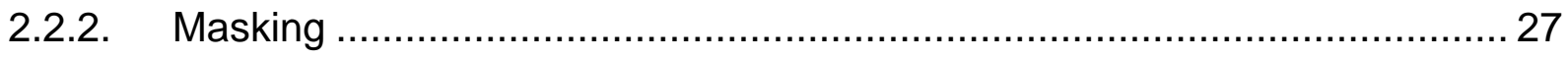

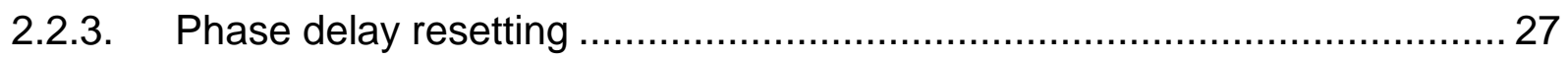

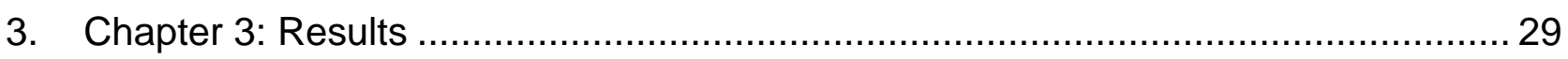

3.1. 'Genetic interaction of Per1 and Dec1/2 in the regulation of circadian locomotor activity' (submitted to Journal of Biological Rhythms) ............................. 29

3.2. 'Advanced Activity Phase Entrainment and Restored Free-Running SCN Rhythms in Per2/Dec Mutant Mice' (submitted to Chronobiology International) ....... 57

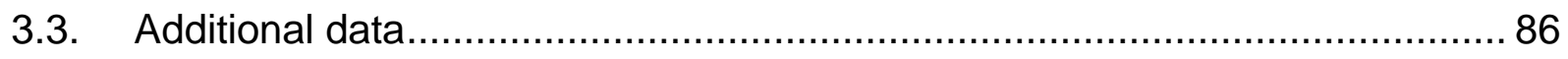

3.3.1. Phase delay responses and photic light suppression in Per1/Dec mutant

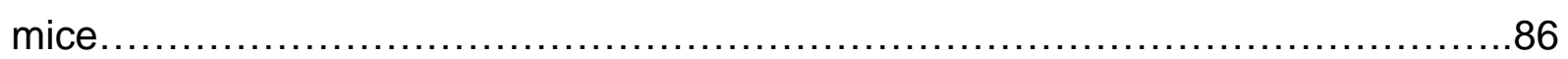

3.3.2. Locomotor activity under constant light in Per2/Dec mutant mice............... 88 
3.3.3. Photic phase delays in Per single mutant mice...................................... 90

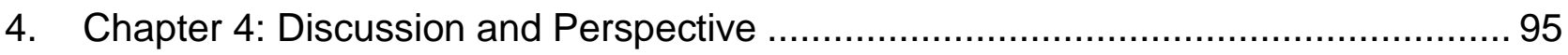

4.1. Synergistic Per1-Dec and Per2-Dec interaction in photic entrainment .......... 95

4.2. Compensation effects cause partial rescue in free-run .............................. 96

4.3. Bimodular transcriptional function of DEC in the mammalian pacemaker ...... 98

4.4. Per2 expression is essential for phase delay resetting ................................. 99

4.5. Potentiated PER1/DEC1 compensation elevates Per1 expression efficiently for

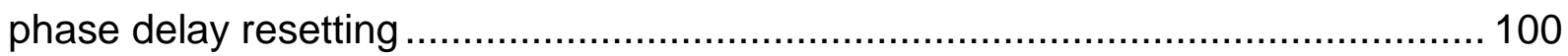

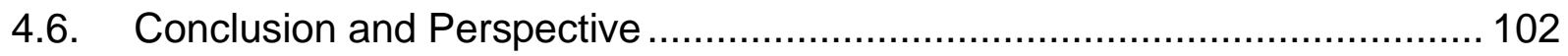

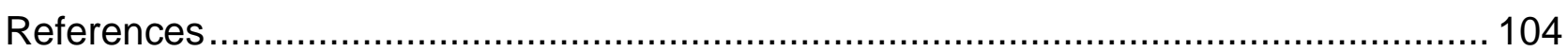

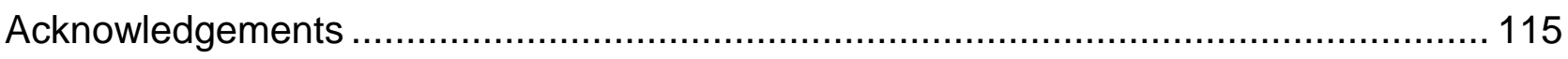

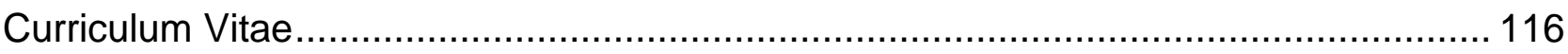




\section{Summary}

Many behavioural and physiological processes in mammals display circadian (24 hour) rhythms controlled by an internal timekeeping system - the circadian clock. The pacemaker of the circadian clock of mammals is located in the suprachiasmatic nuclei (SCN) of the hypothalamus and synchronizes peripheral oscillators in a hierarchical manner to the external light/dark (LD) cycles by humoral and neuronal pathways. The molecular timekeeping machinery consists of a network of transcriptional translational feedback loops (TTL). The mammalian core TTL includes CLOCK/(NPAS2) and BMAL1/(ARNTL) which together activate the transcription of E-box controlled clock genes such as Period (Per1-3), Cryptochrome $($ Cry1,2) and Dec $(1,2)$. PER $(1,2)$ and CRY $(1,2)$ heterodimerize and translocate back to the nucleus where they repress CLOCK/BMAL1 mediated transcription. The basic helix-loop-helix transcription factors DEC1 (BHLHE40) and DEC2 (BHLHE41) can interact with the core TTL by binding to CLOCK/BMAL1 complexes or to E-box elements, forming an accessory feedback mechanism. In Drosophila, the DEC ortholog CWO shows synergistic interaction to PER. This promoted, us to analyze PER(1,2)-DEC interactions in the murine circadian system. We generated $\operatorname{Per}(1,2) / D e c$ double and triple mutant mice and measured circadian locomotor behaviour and clock gene expression in the SCN. Our wheel-running data suggest synergistic Per(1,2)-Dec interactions in photic entrainment with an advanced activity onset indicative of impaired sleep behaviour. Under free-run, the Per1-Dec interactions remain synergistic whereas the Per2-Dec interaction becomes antagonistic together with a partial rescue of the Per2 phenotype. This rescue is seen at multiple levels including period length and rhythmicity of behaviour as well as clock gene expression in the SCN. The molecular data suggest a bimodular regulatory function of $\operatorname{Per}(1,2)$-Dec on E-box controlled clock genes in the SCN, moreover Per1-Dec bimodularity is time-of-day dependent. For the first time, we show that DECs together with PER1 activate the transcription of Bmal1 in the SCN. Investigations of photic phase delay response suggest a model for phase delay resetting with an essential role of Per2/PER2 and a minor role of Per1/PER1. Together, our results show interactions of $\operatorname{Per}(1,2)$ and $\operatorname{Dec}(1,2)$ feedback in the mammalian pacemaker with different modes of interactivities under entrained and free-run conditions together with a compensation effect. These data postulate a conservation of Per-Dec (Cwo) interactions between vertebrate and invertebrate circadian clocks. 


\section{Zusammenfassung}

Viele Verhaltens- und physiologische Prozesse zeigen zirkadiane ( 24 Stunden; von lat. circa dies: „ungefähr ein Tag“) Rhythmen, welche durch interne Uhren reguliert werden. In Säugetieren sitzt der zentrale zirkadiane Schrittmacher im Nucleus suprachiasmaticus (SCN) des Hypothalamus. Von dort werden untergeordnete Uhren in den Organen des Körpers kontrolliert, die dann das Zeitsignal in physiologische Befehle umsetzen. Auf molekularer Ebene basieren zirkadiane Uhren auf einem Netzwerk von transkriptionell-translatorischen Rückkopplungsschleifen (TTL, engl. transcriptional translational feedback loops) aus sog. Uhrengenen, darunter Period (Per1-3), Cryptochrome (Cry1,2), Bmal1 (Arntl) und Clock (Npas2). CLOCK und BMAL1 sind zwei Transkriptionsfaktoren, die über cis-regulatorische E-Box-Promotorelemente die Expression der Period- und Cryptochrome-Gene aktivieren. PER- und CRY-Proteine heterodimerisieren im Zytoplasma und werden in den Zellkern zurückgeführt. Dort inhibieren sie den CLOCK/BMAL1-Komplex - und damit ihre eigene Produktion.

Die Helix-Loop-Helix-Transkriptionsfaktoren DEC1 (BHLHE40) und DEC2 (BHLHE41) interagieren über Bindung an den CLOCK/BMAL1-Komplex oder an E-Box- Sequenzen sowohl inhibitorisch als auch aktivatorisch mit dem zirkadianen TTL. Für Drosophila wurde eine synergistische Interaktion zwischen dem DEC-Ortholog CWO und PER postuliert, welches eine ähnliche Per(1,2)-Dec-Interaktion in der Säugetieruhr vermuten lässt. Um dies zu untersuchen, wurden Per(1,2)/Dec-doppel- und tripel-mutante Mäuse (Mus musculus) generiert, die im Laufrad hinsichtlich ihres zirkadianen Verhaltens als auch auf molekularer Ebene per In Situ-Hybridisierung analysiert wurden. Es zeigte sich, dass synergistische $\operatorname{Per}(1,2)-D e c-I n t e r a k t i o n e n$ während des Entrainments vorliegen. Per-Dec-Doppel- und Tripelmutanten zeigen einen vorgezogenen Aktivitätsbeginn, ähnlich zu Menschen mit „Vorgelagertem Schlafphasensyndrom“ (ASPS, engl. advanced sleep phase syndrome). Unter konstanten Umweltbedingungen ist der Per1-DecSynergismus beibehalten. Allerdings zeigen Per2 und Dec1/2 nun antagonistische Interaktionen. In Per2/Dec-Doppelmutanten zeigt sich zudem ein Rekonstitutionseffekt hinsichtlich Periodenlänge und Rhythmik im Verhalten als auch auf molekularer Ebene im SCN. Weiterhin zeigten die In situ-Daten, dass im SCN eine bimodale Funktion der Per(1,2)-Dec-Interaktion auf die Uhrengenexpression vorliegt, wobei die Per1-Dec Interaktion tageszeitabhängig variiert. Erstmalig konnten wir zudem nachweisen, dass die 
DECs zusammen mit PER1 die Bmal1-Transkription im SCN regulieren. Zusammenfassend lässt sich sagen, dass Gen-spezifische, tageszeit- und lichtabhängige funktionelle Interaktionen zwischen den $\operatorname{Per}(1,2)$ - und $\operatorname{Dec}(1,2)$-Rückkopplungsschleifen im zirkadianen Uhrensystem der Säugetiere vorliegen. Die PER-CWO-Interaktion in der Uhr der Fliege scheint im TTL der Säugetiere weitestgehend konserviert. 


\section{Abbreviations}

\begin{tabular}{|c|c|}
\hline${ }^{\circ} \mathrm{C}$ & degree Celsius \\
\hline$\mu \mathrm{m}$ & micrometer \\
\hline${ }^{35} \mathrm{~S}-U T P$ & sulphur-35 labeled uridinetriphosphate \\
\hline $3 \mathrm{~V}$ & third ventricle \\
\hline $5-\mathrm{HT}$ & serotonin \\
\hline A & adenine \\
\hline ACTH & adrenocorticotropic hormone \\
\hline AMPAR & a-amino-3-hydroxy-5-methyl-4-isoxazolepropionic acid \\
\hline ASPS & advanced sleep phase syndrome \\
\hline AVP & arginine vasopressin \\
\hline bHLH & basic heix-loop-helix \\
\hline Bmal1 & Brain-and-muscle-arntl-like protein 1 \\
\hline BPD & bipolar disorder \\
\hline C & cytosine \\
\hline Calb & calbindin \\
\hline cAMP & cyclic adenosine monophosphate \\
\hline CCG & clock-controlled gene \\
\hline cDNA & complementary DNA \\
\hline CK1 & casein kinase 1 \\
\hline Clock & circadian locomotor output cycles kaput \\
\hline CMV & human cytomegalovirus \\
\hline CNS & central nervous system \\
\hline $\mathrm{CO}$ & carbon monoxide \\
\hline CRE & cAMP response element \\
\hline CREB & cAMP response element binding protein \\
\hline Cry & Cryptochrome \\
\hline CT & Circadian time \\
\hline cwo & clockwork orange \\
\hline Dbp & D-box binding protein \\
\hline DD & constant darkness \\
\hline DFG & German Research Foundation \\
\hline $\mathrm{dm}$ & dorsomedial \\
\hline $\mathrm{DMH}$ & dorsomedial nucleus of the hypothalamus \\
\hline DNA & deoxyribonucleic acid \\
\hline DRN & dorsal raphe nucleus \\
\hline DSPS & delayed sleep phase syndrom \\
\hline e.g. & for example \\
\hline E4bp4 & E4 promoter-binding protein 4 \\
\hline
\end{tabular}


engl.

$\mathrm{F} 1$ or F2

FAA

FASPS

FBXL3

FEO

Fig.

G

GABA

$\mathrm{GH}$

GHT

Glu

GRP

h

HA

HEK293

i.e.

IGL

ipRGCs

ISH

lat.

LD

LL

LP

luc

MAP

MDD

mEnk

$\min$

MPO

MRN

n.a.

n.i.

NAD

NAMPT

NMDAR

NO

Npas2

NYP

OP

$\mathrm{P}$
English

generation after the parent generation

food anticipatory activity

familial advanced sleep phase syndrome

F-box and leucine-rich repeated protein 3

food-entrainable oscillator

Figure

guanine

gamma-aminobutyric acid

growth hormone

geniculate-hypothalamic tract

glutamate

gastin-releasing peptide

hour

human influenza hemagglutinin

Human Embryonic Kidney 293 cells

id est

intergeniculate leaflet

intrinsically photosensitive retinal ganglion cells

in situ hybridisation

Latin

light/dark

constant light

light pulse

luciferase

mitogen-activated protein

major depressive disorder

met-Enkephalin

minute

medial preoptic region

median raphe nucleus

not analysed

no genetic interaction

nicotinamide adenine dinucleotide

nicotinamide phosphorybosyltransferase

N-Methyl-D-Aspartate receptor

nitric oxide

neuronal PAS domain protein 2

neuropeptide $Y$

optic chiasm

parent generation 
p.m.

PACAP

PAS

PCR

Per

PFA

PK2

PKA

PKCa

PLM

PRC

PVN

RHT

RLS

RNA

RORE

ROR $\alpha$

$S$

SAD

SEM

siRNA

$T$

TGF $\alpha$

TTL

VIP

vl

vol.

WT

ZT

T post meridiem (engl. after midday)

pituitary adenylate cyclase activating polypeptide

Period-Arnt-Single-minded

polymerase chain reaction

Period

paraformaldehyde

prokineticin 2

protein kinase $\mathrm{A}$

protein kinase $\mathrm{C}$ a

periodic limb movements

phase response curve

paraventricular nucleus

retino-hypothalamic tract

restless legs syndrome

ribonucleic acid

retinoic acid-related orphan receptor response element

retinoid-related orphan receptor $\alpha$

supplement

seasonal affective disorder

standard error of the mean

small interfering RNA

thymine

transforming growth factor $\alpha$

transcriptional/translational feedback loop

vasoactive intestinal polypeptide

ventrolateral

volume

wild-type

Zeitgeber time

tau, period lenght 


\section{Chapter 1: Introduction}

Most organisms living on the earth are under the steady influence of daily changes resulting from the rotation of the planet around its axis. During evolution, organisms have adapted their physiological and behavioural parameters to these periodic environmental changes by developing internal mechanisms to determine the time of day - circadian clocks (from circa (lat.) - approximately, dies (lat.) - day) (Ouyang, Andersson et al. 1998).

\subsection{Circadian rhythms}

In 1729 the French astronomer Jean Jacques Ortous de Mairan wrote the first scientific report on endogenously driven biological rhythms with periods of approximately 24 hours (h). He observed the daily leaf movements of Mimosa pudica when the plants were kept in a closed cupboard. Since then circadian rhythms have been observed at all levels of biological organisation, from behavioural changes in mammals, flies and fish to the activity of enzymes and the transcription of specific genes (Pittendrigh 1993).

Endogenous circadian clocks share certain properties including self-sustainment, temperature compensation and the ability of entrainment (or synchronisation) in a timedependent manner by environmental stimuli - termed Zeitgebers ('time givers' in German). Circadian clocks maintain a stable endogenous period of approximately $24 \mathrm{~h}$, even in absence of external Zeitgebers. Because of a deviation of the internal period from $24 \mathrm{~h}$ (see below) the internal timing system has to be reset (shifted) every day by a few minutes to stay in synchrony with the environmental time. The main Zeitgeber for the mammalian clock is light, but there are other, non-photic, Zeitgebers like food, drugs and social interactions. In the absence of external time cues, i.e. under constant lighting conditions (constant darkness (DD) or constant light (LL)), behavioural and physiological rhythms begin to free-run with a period slightly different to $24 \mathrm{~h}$. In mice, a well-established readout for circadian rhythms is locomotor activity, which can be measured by counting running-wheel revolutions. Behavioural activity can be visualized as an actogram (Figure 1) in which wheel revolutions (represented in black dots) are 
plotted against time. A double-plotted actogram shows two days per horizontal line. Day 2 is repeated on the next horizontal line together with day 3 (and so on). Under alternating light/dark (LD) cycles, the locomotor activity of a mouse shows an alteration of $12 \mathrm{~h}: 12 \mathrm{~h}$ rest (called rho) and active (alpha) periods. If the internal period $(r, t a u)$ is exactly $24 \mathrm{~h}$ the animal is entrained to the external LD cycle. The LD cycle is measured in Zeitgeber times (ZT) based on the period of the Zeitgeber (in this case the light regime). ZTO is defined as the time of 'lights on' and ZT12 as time of 'lights off'.

In the absence of a Zeitgeber, the circadian day is portioned into two phases: subjective day and subjective night, which correspond to the rest and active phase, respectively, for nocturnal species. The circadian day is scaled in 24 circadian hours (Circadian time; CT). Under constant conditions, the beginning of the subjective day is defined as CTO (= CT24) and the beginning of the subjective night is CT12 (the onset of activity in nocturnal mice).

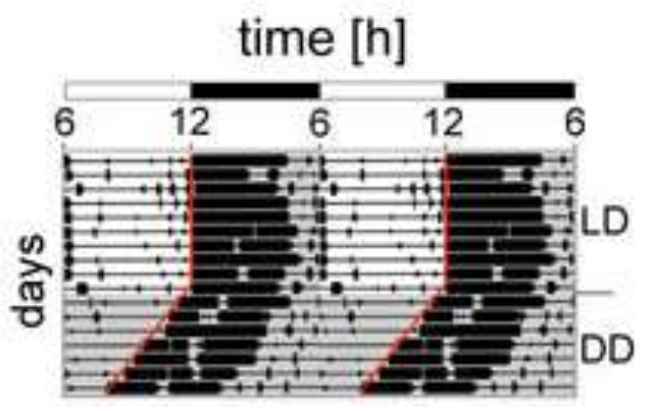

Figure 1: Actogram. Double plotted actogram of mouse locomotor activity (represented in black vertical bars). The mouse is entrained to a $12 \mathrm{~h}: 12 \mathrm{~h}$ LD cycle and after 10 days released into constant dark conditions (DD, dark phases marked in grey). In DD, the activity rhythm starts to free-run with a period of $23.5 \mathrm{~h}$. ZTO in LD corresponds to 6:00 p.m. The red lines indicate the onset of locomotor activity. Modified from (Jud, Schmutz et al. 2005)

\subsubsection{Physiological circadian rhythms}

The circadian oscillator controls diverse physiological processes such as the sleep/wake cycle, daily rhythms in energy metabolism, body temperature and hormone release (Saper, Cano et al. 2005). Blood concentrations of many hormones show circadian rhythms (Hastings, O'Neill et al. 2007). For instance, the hypothalamus sends circadian signals to the pituitary gland that releases hormones in a circadian fashion such as prolactin, adrenocorticotropic hormone (ACTH) and growth hormone (GH) that, in turn, stimulate peripheral endocrine glands like the thyroid, gonads, and the adrenal gland which themselves rhythmically release hormones. For example, ACTH stimulates the circadian synthesis of glucocorticoids in the adrenal glands (Reinberg and Smolensky 1982). The peak of glucocorticoid release correlates with the beginning of the activity phase, i.e. in the early morning in diurnal and in the early night in nocturnal animals 
(Kalsbeek, Kreier et al. 2007). Glucocorticoids have many different functions in body homeostasis and stress responses (Sage, Maurel et al. 2001; Kalsbeek, Ruiter et al. 2003; Atkinson, Wood et al. 2006; Papadimitriou and Priftis 2009; So, Bernal et al. 2009). Furthermore, melatonin synthesis in the pineal gland is under circadian control. Plasma melatonin levels are high during the night and low during the day. Melatonin is involved in sleep/wake cycle regulation (Cajochen, Krauchi et al. 2003) and also modulates cortisol secretion from the adrenal gland.

Various enzymes of metabolic pathways such as glycolysis, gluconeogenesis, cholesterol and lipid metabolism are under circadian control (Panda, Antoch et al. 2002), suggesting a strong link between the circadian system and metabolism. The circadian timekeeping system drives rhythmic metabolic processes and, vice versa, various metabolic parameters affect the clock (reviewed by Green, Takahashi et al. 2008). Thus, it is not surprising that deregulation of the circadian system can contribute to metabolic diseases (see below).

A further physiological aspect regulated by the circadian clock is the immune system, which consists of innate components and adaptive components ( $T$ and $B$ lymphocytes). It was shown that the circadian system regulates immune responses in humans (Levi, Canon et al. 1988). The proliferative activity of circulating $\mathrm{T}$ - and B-cells in the peripheral blood varies throughout the day (Levi, Canon et al. 1988). T-cell response and proliferation is most efficient in the morning; B-cells, however, show higher reactivity in the evening (Deshmukh 2006).

\subsection{The mammalian clock system}

The circadian clock system consists of three main parts: 1) a central pacemaker which generates clock rhythms, 2) an input pathway which resets the clock to the outside environment by photic as well as non-photic Zeitgebers, and 3) clock output pathways to efferent systems.

\subsubsection{The suprachiasmatic nucleus (SCN)}

In 1972, two independent groups identified the pacemaker of the mammalian circadian clock in the ventrolateral hypothalamus - the suprachiasmatic nuclei (SCN). Lesion of 
these structures results in a loss of corticosterone secretion (Moore and Eichler 1972) and of drinking and locomotor activity rhythms (Stephan and Zucker 1972). Further SCN lesions in rats show disrupted circadian rhythms of melatonin secretion (Klein and Moore 1979) suggesting a SCN-mediated regulation of melatonin production in the pineal gland.
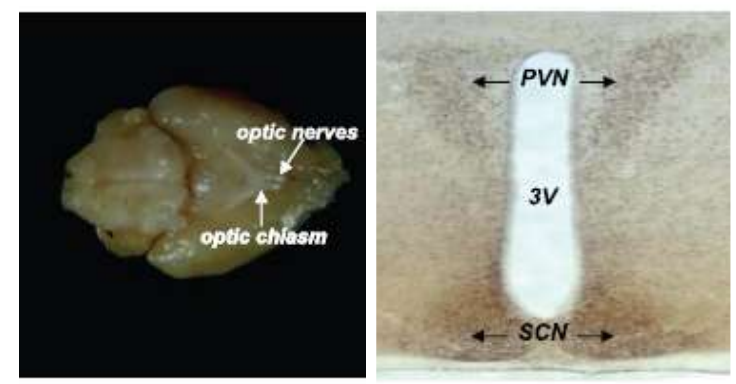

Figure 2: Anatomical view of the SCN. Left picture shows a ventral view on the mouse brain. Right picture shows light-induced $c$-Fos expression in the ventromedial hypothalamus. Modified from (Golombek and Rosenstein 2010)

The bilateral SCN consist of approximately 10,000 heterogeneous neurons. They are located dorsal to the optic chiasm on either side of the third ventricle (Figure 2). The two main subdivisions of the SCN are the ventrolateral area (vISCN or 'core') and the dorsomedial region (dmSCN or 'shell') (Moore, Speh et al. 2002). The core SCN sits adjacent to the optic chiasm and contains neurons that synthesize vasoactive intestinal polypeptide (VIP) and gastrin-releasing peptide (GRP). The dorsomedial SCN expresses arginine vasopressin (AVP), calbindin (Calb) and met-Enkephalin (mEnk) (Golombek and Rosenstein 2010; Figure 3).

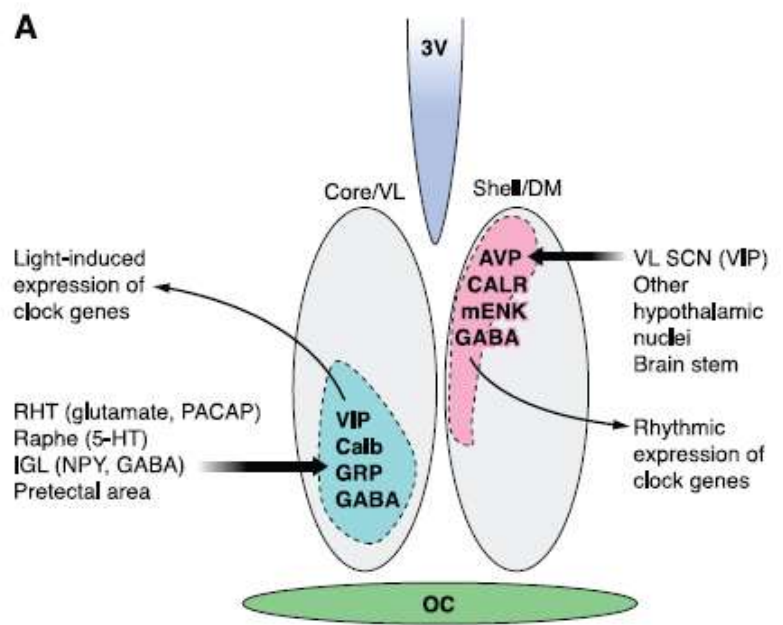

Figure 3: Subdivisions of SCN. The left SCN represents the neurochemical subdivision of the ventrolateral SCN, which is connected to the RHT, the raphe nuclei and the IGL. The right SCN shows the neurotransmitter subdivision of the dorsomedial $\mathrm{SCN}$, which receives innervation from the ventrolateral SCN and other hypothalamic areas. $\mathrm{RHT}$, retino-hypothalamic tract; $3 \mathrm{~V}$, third ventricle; $\mathrm{OC}$, optic chiasm; IGL, intergeniculate leaflet; VL, ventrolateral; DM, dorsomedial; VIP, vasoactive intestinal polypeptide; Calb, calbindin; GRP, gastrinreleasing peptide; AVP, arginine vasopressin; CALR, calretinin; mENK, met-Enkephalin. Modified from (Golombek and Rosenstein 2010)

In 1979, Inouve et al. demonstrated that SCN neurons are able to maintain circadian rhythms of spontaneous electrical activity even in isolation from other brain structures (Inouye and Kawamura 1979). Moreover, these rhythms are maintained ex vivo such as in 
SCN slices and in dissociated SCN cells (Green and Gillette 1982; Shibata, Oomura et al. 1982; Welsh, Logothetis et al. 1995; Yamaguchi, Isejima et al. 2003). Neuropeptide signaling including VIP (through VPAC receptors) and GRP-mediated communication is necessary for neuronal synchronization within the SCN (Maywood, Reddy et al. 2006; Maywood, O'Neill et al. 2007; Hughes, Guilding et al. 2008). Others proposed that intraSCN synchronizing mechanisms include nitric oxide (NO) and gamma-aminobutyric acid (GABA) neurotransmission (Liu and Reppert 2000; Albus, Vansteensel et al. 2005; Aton, Colwell et al. 2005; Vosko, Schroeder et al. 2007).

Taken together, the SCN contain autonomous cellular oscillators, which are synchronized by neuronal and neuropeptide signalling. The oscillatory coupling of the SCN neurons is essential for the nuclei to act as a pacemaker (Liu, Welsh et al. 2007).

\subsubsection{Clock input}

The SCN clock can be reset via three different pathways: 1) the retino-hypothalamic tract $(\mathrm{RHT}), 2)$ the geniculate-hypothalamic tract $(\mathrm{GHT})$ and 3) serotonergic $(5-\mathrm{HT})$ input from the dorsal raphe nucleus (DRN) and median raphe nucleus (MRN) (Dibner, Schibler et al. 2010). The RHT pathway transmits photic information from the eye (Moore and Lenn 1972), whereas the GHT and the raphe provide non-photic input to the SCN (Figure 4).
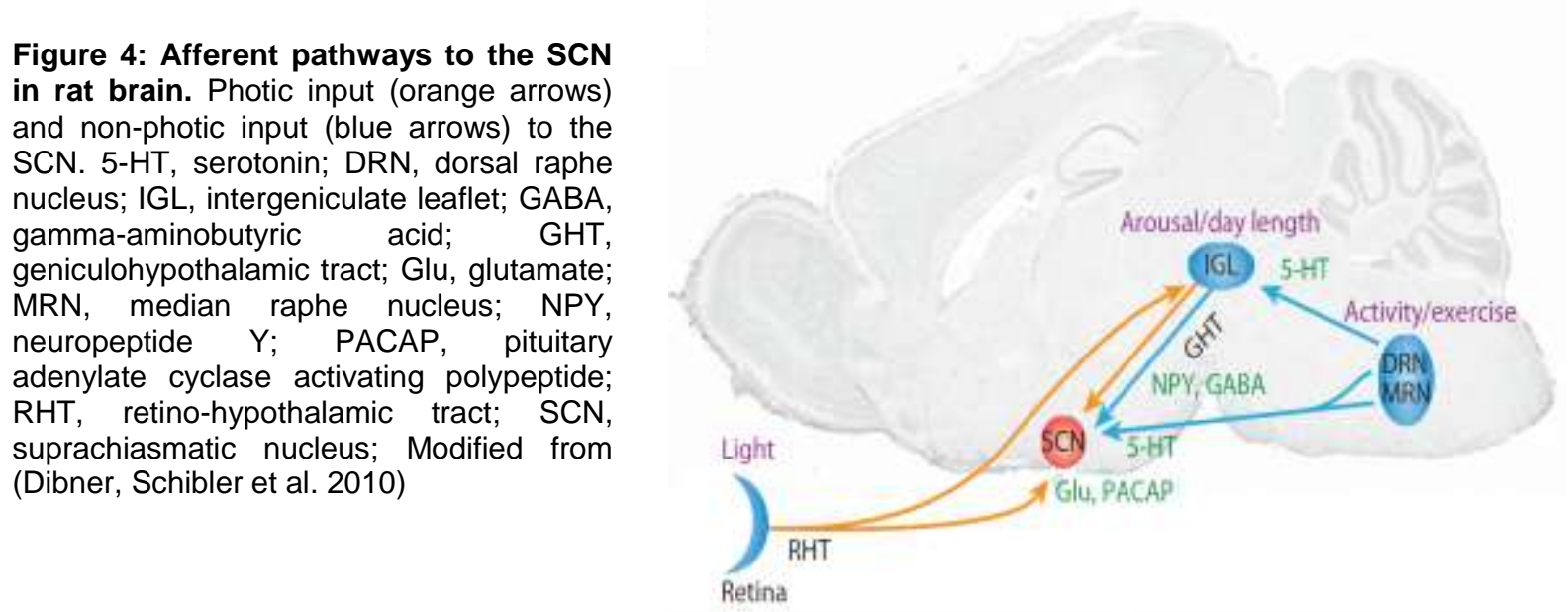

The classical retinal photoreceptors are not critical for circadian synchronization as mice with ablation of rods and cones still entrain to light (Freedman, Lucas et al. 1999; Lucas, Freedman et al. 2001). However, in the additional absence of the retinal photopigment melanopsin which is expressed in a subset of directly light sensitive ganglion cells (ipRGCs, intrinsically photosensitive retinal ganglion cells) (Provencio, 
Rollag et al. 2002) mice fail to entrain to light/dark cycles and show abolished masking responses to light (Hattar, Lucas et al. 2003). Thus, melanopsin containing ipRGCs act as photic time sensors for the SCN clock.

The RHT transmits the light information from the ipRGCs via monosynaptic projections (Berson 2007) to the ventrolateral part of the SCN (SCN core), signalling via the neurotransmitters glutamate (Glu) and pituitary adenylate cyclase activating polypeptide (PACAP) (Figure 5; Ebling 1996; Hannibal, Ding et al. 1997; Hannibal 2002; Hirota and Fukada 2004; Hannibal 2006). Cells in the SCN express different glutamate (AMPAR and NMDAR) (Gannon and Rea 1994; Mick, Yoshimura et al. 1995; Ebling 1996; Mizoro, Yamaguchi et al. 2010) and PACAP receptors (Hannibal 2002). Neuronal stimulation of the SCN results in activation of intracellular signalling cascades including calciumdependent kinases and proteases (calmodulin, MAP kinases and PKA) (Obrietan, Impey et al. 1998; Yokota, Yamamoto et al. 2001; Hirota and Fukada 2004; Antle, Smith et al. 2009). Ultimately the transcription factor cAMP response element binding protein (CREB) is phosphorylated (Ginty, Kornhauser et al. 1993; Gau, Lemberger et al. 2002) and induces the expression of clock genes, e.g. Per1 (Shigeyoshi, Taguchi et al. 1997; Yan and Silver 2002), Per2 (Shearman, Zylka et al. 1997; Yan and Silver 2002) and Dec1 (Honma, Kawamoto et al. 2002) via binding to cAMP response element (CRE) sequences in the respective promoters (Gillette and Tischkau 1999; Obrietan, Impey et al. 1999; Hirota and Fukada 2004; Antle and Silver 2005).
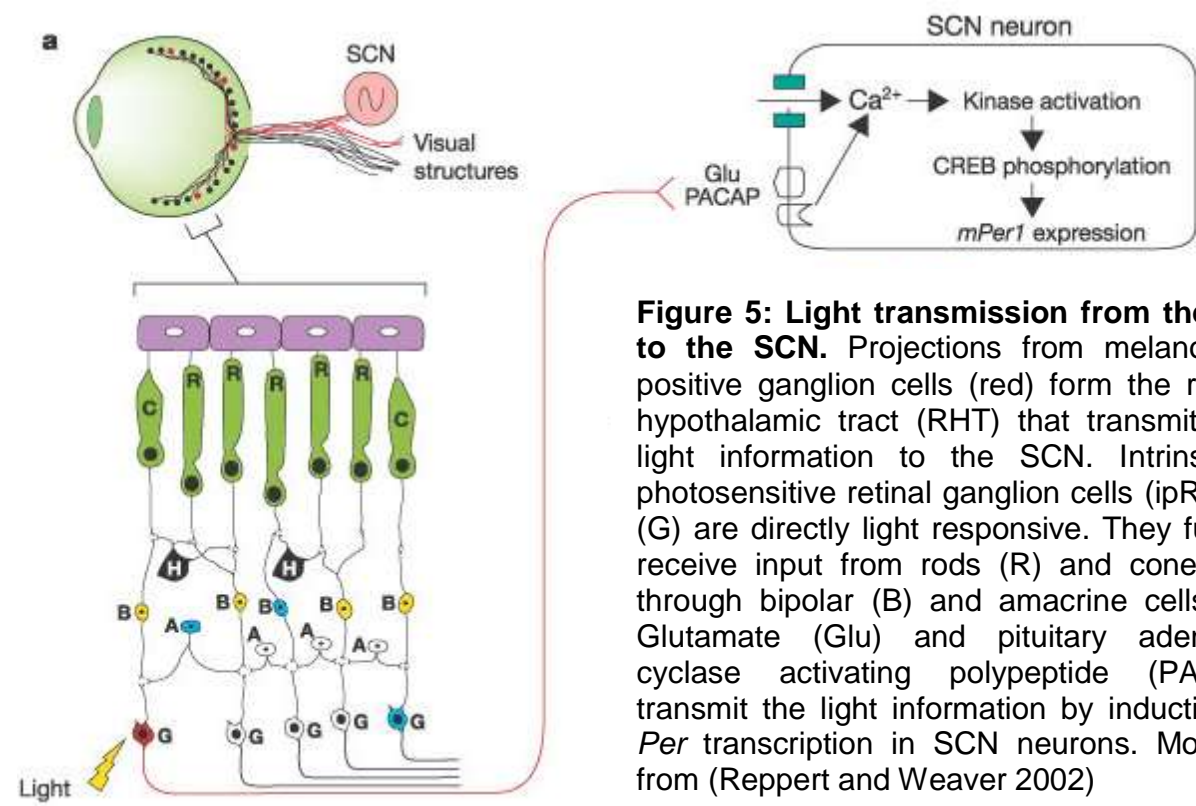

Figure 5: Light transmission from the eye to the SCN. Projections from melanopsinpositive ganglion cells (red) form the retinohypothalamic tract $(\mathrm{RHT})$ that transmits the light information to the SCN. Intrinsically photosensitive retinal ganglion cells (ipRGCs) (G) are directly light responsive. They further receive input from rods $(R)$ and cones $(C)$ through bipolar $(B)$ and amacrine cells $(A)$. Glutamate (Glu) and pituitary adenylate cyclase activating polypeptide (PACAP) transmit the light information by induction of Per transcription in SCN neurons. Modified from (Reppert and Weaver 2002) 
Interestingly, the RHT projects not only to the SCN but also to the intergeniculate leaflet (IGL). The IGL relays photic signals to the $S C N$ via the GHT based on neuropeptide $Y$ (NPY) and GABA neurotransmission (Harrington, Nance et al. 1985; Moore and Card 1994; Jacob, Vuillez et al. 1999; Juhl, Hannibal et al. 2007).

In addition to light so called non-photic Zeitgebers exist (Hastings, Duffield et al. 1997) such as behavioural arousal (e.g. dark pulses during the light phase, novel wheel access; Reebs and Mrosovsky 1989; Van Reeth and Turek 1989), timed food availability (Mistlberger 1994; Stephan 2002; Feillet, Albrecht et al. 2006; Mendoza 2007), social contacts (Mrosovsky 1988; Mrosovsky, Reebs et al. 1989) and certain drugs (e.g. benzodiazepine, morphine; Van Reeth and Turek 1989; Marchant and Mistlberger 1995; Mrosovsky 1996). Figure 4 shows afferent projections to the SCN from different brain regions, such as the NPY system from the IGL and 5-HT input from the midbrain raphe which together mediates non-photic entrainment by behavioural arousal (Mrosovsky 1996; Hastings, Duffield et al. 1998). Further, mice can also entrain to daily food restriction, showing food anticipatory activity (FAA) prior to expected mealtime. FAA is represented by increase locomotion, corticosterone secretion and body temperature (Mistlberger 1994). The localisation and the molecular mechanism of this food-entrainable oscillator (FEO) are still unclear. Importantly, FAA is preserved in SCN-ablated animals (Stephan 2002).

\subsubsection{Clock output}

The central clock uses a dual control to coordinate daily rhythmicity in the periphery neuronal and humoral pathways (Buijs and Kalsbeek 2001). SCN transplantation experiments show that the SCN generate the rhythmicity for the organism. The group of Menaker found that SCN transplantation restores circadian rhythms to arrhythmic animals with period of the donor genotype (Ralph, Foster et al. 1990). Other experiments show that the neurotransmitters GABA and glutamate are also crucial for transmission of SCN outputs (Hermes, Coderre et al. 1996). SCN neurons send efferent projections to other brain regions, such as the paraventricular nucleus (PVN), the medial preoptic region (MPO) and the dorsomedial nucleus of the hypothalamus (DMH) (Figure 6; see above) (Kalsbeek, Palm et al. 2006; Dibner, Schibler et al. 2010; Nader, Chrousos et al. 2010). 
Placing SCN transplants encapsulated in a semipermeable membrane into the third ventricle restores behavioural rhythms in SCN-lesioned rats (Silver, LeSauter et al. 1996), postulating that diffusible molecules released by the transplant might be sufficient to restore rhythmicity without the need for synaptic connections. The current evidence suggests that the SCN also uses several humoral mediators as output signals such as argenine vasopressin (AVP) (Kalsbeek, Buijs et al. 1992), prokineticin 2 (PK2) (Cheng, Bullock et al. 2002), vasoactive intestinal polypeptide (VIP) (Kalsbeek and Buijs 1992) and transforming growth factor a (TGFa) (Kramer, Yang et al. 2001; Li, Sankrithi et al. 2002).

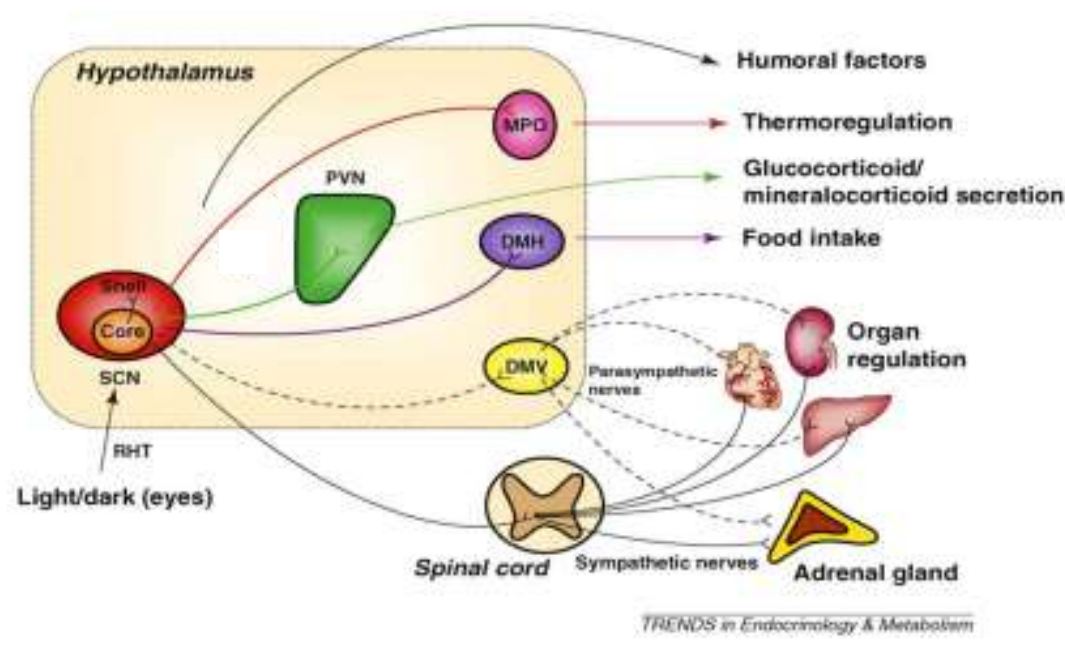

Figure 6: Neuronal and humoral pathways mediate the synchronization of peripheral rhythms. The SCN receives light information from the retina through the RHT. This time information is transmitted via efferent neurons from the $\mathrm{SCN}$ to other brain regions such as PVN, DMH and MPO which regulate glucocorticoid secretion, thermoregulation and food intake. PVN paraventricular nucleus; $\mathrm{DMH}$, dorsomedial nucleus of the hypothalamus; MPO, medial preoptic region; modified from (Nader, Chrousos et al. 2010)

\subsubsection{The molecular clockwork}

\subsubsection{Transcriptional/translational feedback loops}

The molecular basis of the circadian clock are interconnected transcriptional/translational feedback loops (TTL) that drive rhythmic expression of core clock components (Figure 7). These are defined as genes/proteins that are necessary for the generation and regulation of circadian rhythms within individual cells throughout the organism. In 1971, the first clock gene, Period, was identified in a mutagenesis screen in Drosophila melanogaster (Konopka and Benzer 1971). Since then other clock genes have been found in different model organisms such as cyanobacteria, fungi, flies, hamsters and mice as well as humans. These observations revealed that the molecular structure of 
circadian clocks is evolutionary highly conserved (Rosbash 2009; Zhang and Kay 2010). Below, I will focus on the components of the mammalian TTL.

The positive limb of the mammalian core feedback loop includes members of the basic helix-loop-helix (bHLH)-Period-Arnt-Single-minded (PAS) transcription factor family: circadian locomotor output cycles kaput (CLOCK), its paralog neuronal PAS domain protein 2 (NPAS2) and their partner brain-and-muscle-Arnt-like protein 1 (BMAL1). CLOCK(NPAS2)/BMAL1 heterodimers activate the transcription of cis-regulatory E-box containing target genes including Period (Per1-3) and Cryptochrome (Cry1,2) genes (King, Zhao et al. 1997; Gekakis, Staknis et al. 1998; Hogenesch, Gu et al. 1998; Kume, Zylka et al. 1999; Bunger, Wilsbacher et al. 2000; Zheng, Albrecht et al. 2001). Negative feedback is achieved by the heterodimerization of PER and CRY proteins which together are translocated back to the nucleus and repress CLOCK(NPAS2)/BMAL1 mediated transcription (Figure 7) (Griffin, Staknis et al. 1999; Kume, Zylka et al. 1999; Okamura, Miyake et al. 1999; Shearman, Sriram et al. 2000; Lee, Etchegaray et al. 2001; Sato, Yamada et al. 2006), thereby shutting down their own expression.

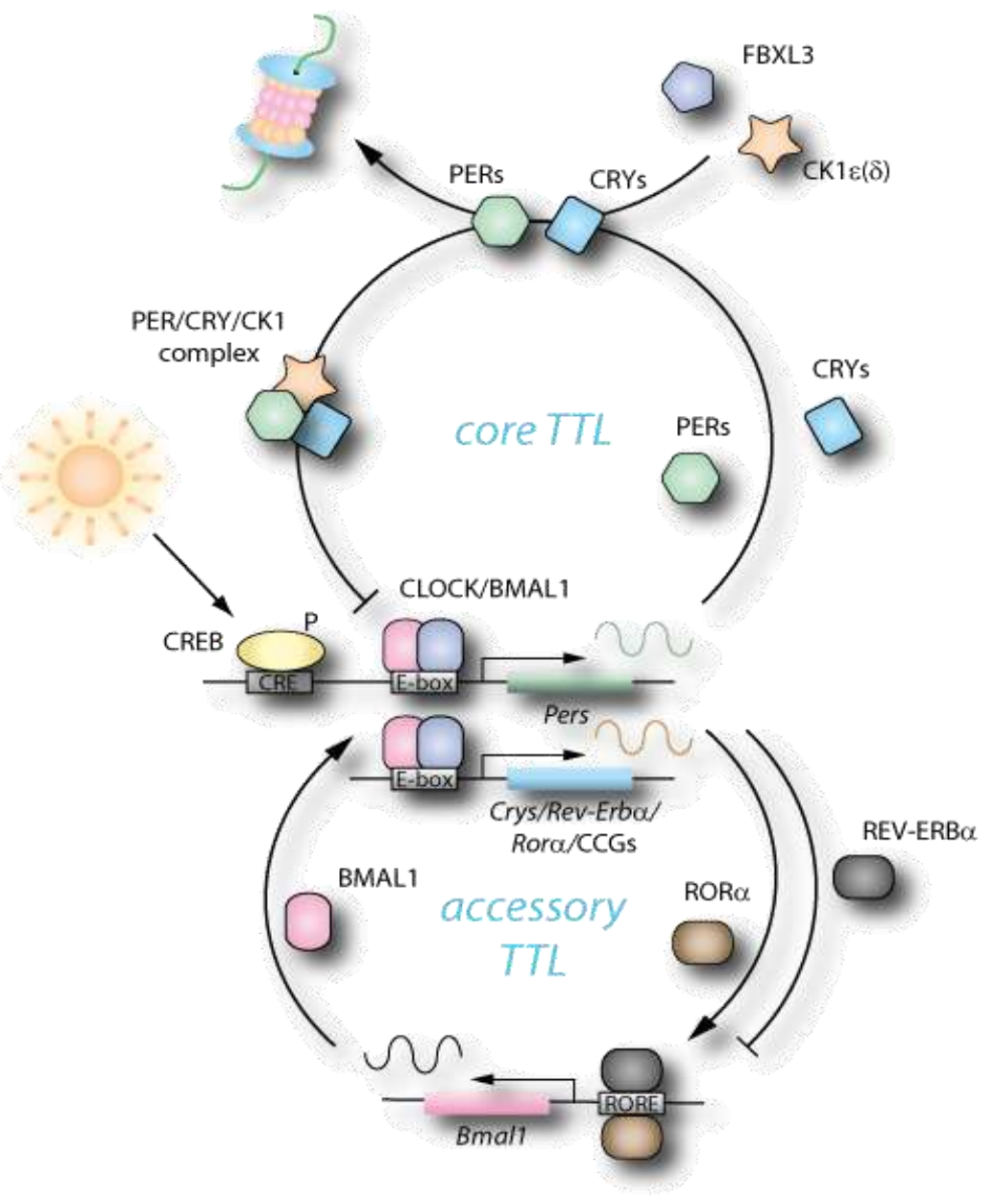

Figure 7:

Transcriptional/translational feedback loops (TTLs) of the mammalian circadian clock. The CLOCK/BMAL1 complex activates the transcription of $\mathrm{E}$ box containing clock (such as Per, Cry, Rev-Erba and RORa) and clock-controlled genes (CCGs). PER and CRY heterodimerize and inhibit CLOCK/BMAL1-activated transcription. REV-ERBa and RORa form negative and positive feedbacks on Bmal1 expression, respectively. Casein kinase $1 \varepsilon(\delta) \quad(C K 1 \varepsilon(\delta))$ and FBXL3 regulate the degradation of the PER/CRY complex. RORE, retinoic acid-related orphan receptor response element. Modified from (Oster 2006) 
This autoregulatory feedback loop takes approximately $24 \mathrm{~h}$ to complete. Additionally, post-translational modifications such as phosphorylations and ubiquitinations regulate the period of the clock oscillator. Casein kinase (CK) $1 \varepsilon$ and $1 \delta$ phosphorylate the clock proteins PER1, PER2, CRY1 and CRY2 (Akashi, Tsuchiya et al. 2002; Eide, Vielhaber et al. 2002) while F-box and leucine-rich repeat protein 3 (FBXL3) ubiquitinates CRY1 and CRY2 (Busino, Bassermann et al. 2007; Godinho, Maywood et al. 2007; Siepka, Yoo et al. 2007), negatively regulating PER $1 / 2$ and CRY1/2 accumulation by marking them for proteasomal degradation (Figure 7).

In addition to the core loop there are several accessory loops, one of which involves transcription of the orphan nuclear receptors Rev-Erba and Rora (Preitner, Damiola et al. 2002; Sato, Panda et al. 2004; Triqueneaux, Thenot et al. 2004; Akashi and Takumi 2005). REV-ERBa and RORa proteins compete for binding to retinoic acid-related orphan receptor response elements (ROREs) on the Bmal1 promoter and repress or activate Bmal1 transcription, respectively (Figure 7) (Preitner, Damiola et al. 2002; Ueda, Chen et al. 2002; Sato, Panda et al. 2004; Triqueneaux, Thenot et al. 2004; Akashi and Takumi 2005; Guillaumond, Dardente et al. 2005). An additional accessory loop is mediated via D-boxes, which are present in promoters of the Per genes. D-box-binding protein (DBP) and E4 promoter-binding protein 4 (E4BP4) act as activators or repressors, respectively, of the transcription of $\mathrm{D}$-box containing genes. Dbp transcription itself is $\mathrm{E}$-box regulated, whereas transcription of E4bp4 is regulated by a RORE in its promoter.

Hundreds of rhythmic clock controlled genes (CCGs) are regulated by E-boxes, ROREs or D-boxes in their promoter regions. The phase of regulation depends on the combination of these elements on specific promoters and is highly tissue-specific, reflecting the physiological function of the tissue. In most tissues up to $10 \%$ of the whole transcriptome is rhythmically expressed (Panda, Antoch et al. 2002; Storch, Lipan et al. 2002; Panda and Hogenesch 2004).

Additional genes were discovered that influence the molecular machinery of the circadian system. A recent genome-wide small interfering RNA (siRNA) screen showed that more than 200 genes might play a role in the clock biology regulating amplitude and period of the cellular clock (Zhang, Liu et al. 2009). Thus, the circadian system appears more complex than presented in Figure 7. Further, it seems that clock genes are also affected by the metabolic state of a cell (reviewed by Kovac, Husse et al. 2009). Carbon monoxide (CO) inhibits DNA binding of NPAS2 and REV-ERBa (Dioum, Rutter et al. 2002) whereas REV-ERBa acts as sensor for heme which controls their DNA binding 
activity (Kaasik and Lee 2004; Yin, Wu et al. 2007). Further, the DNA binding activity of CLOCK/BMAL1 heterodimers is dependent on the redox state of the cell (Rutter, Reick et al. 2001). The inhibition of the NAD biosynthesis enzyme nicotinamide phosphorybosyltransferase (NAMPT) promotes oscillation of Per2 transcription by releasing CLOCK/BMAL1 from suppression by SIRT1 (Nakahata, Sahar et al. 2009; Ramsey, Yoshino et al. 2009).

\subsubsection{Dec1 and Dec2}

Dec1 (Bhlh40, Sharp2, Stra13, Clast5) and Dec2 (Bhlh41, Sharp1) were identified as cAMP-inducible genes in a culture system of human embryonic chondrocytes and by searching a data base for new bHLH transcription factor family members (Shen, Kawamoto et al. 1997; Fujimoto, Shen et al. 2001). Both DEC proteins are closely related (Fujimoto, Shen et al. 2001), but human DEC1 (hDEC1, 412 amino acids) is shorter than hDEC2 (482 amino acids). The mouse Dec genes are located on the same chromosome (Dec1: chr. 6 E-F1; Dec2: chr. 6 G2-G3).

DEC1 and DEC2 are regulators of different biological processes including embryonic development, cell differentiation, cell growth, immune function and hormone expression (Yamada and Miyamoto 2005). Moreover, the expression of Dec1 and Dec2 genes shows circadian rhythms in the SCN, several other brain regions and in the periphery (Honma, Kawamoto et al. 2002; Noshiro, Furukawa et al. 2005). Dec transcription is activated by CLOCK(NPAS2)/BMAL1 heterodimers binding to E-boxes in the Dec promoters and light exposure acutely induces Dec1 transcription in SCN neurons (Honma, Kawamoto et al. 2002; Butler, Honma et al. 2004; Rossner, Oster et al. 2008). These data indicate that the Decs/DECs are involved in the circadian TTL. It was shown that DEC1 as well as DEC2 act as transcriptional regulators by competition with CLOCK/BMAL1 complexes for the cis-regulatory E-box elements, or by direct protein-protein interaction with BMAL1 (Honma, Kawamoto et al. 2002; Sato, Kawamoto et al. 2004) (Figure 8). Both DECs negatively regulate their own transcription in vitro (Honma, Kawamoto et al. 2002; Azmi, Sun et al. 2003; Li, Xie et al. 2003; Hamaguchi, Fujimoto et al. 2004; Kawamoto, Noshiro et al. 2004; Li, Song et al. 2004) and have tissue-dependent regulatory functions as coactivators or co-repressors at E-box sites (Rossner, Oster et al. 2008). In vitro, ovine DEC1 has bidirectional transcriptional function as an activator or repressor of Per1 and Rev-Erba transcription, respectively (Dardente, Fustin et al. 2009). In vivo analyses 
suggest a role of DEC1 in circadian output regulation in the periphery (Grechez-Cassiau, Panda et al. 2004) where it modulates the phase of clock gene expression (Nakashima, Kawamoto et al. 2008). Further, studies on Dec1/2 mutant mice suggest that the Decs have redundant functions in the mammalian circadian clock (see below) (Rossner, Oster et al. 2008). In Drosophila, the Dec ortholog clockwork orange (cwo) shows similar transcriptional regulatory functions, and synergistic interactions with PER in the timekeeping mechanism were postulated (Kadener, Stoleru et al. 2007; Lim, Chung et al. 2007; Matsumoto, Ukai-Tadenuma et al. 2007; Richier, Michard-Vanhee et al. 2008), indicative of an analogue PER-DEC synergistic interaction in the mammalian circadian system.

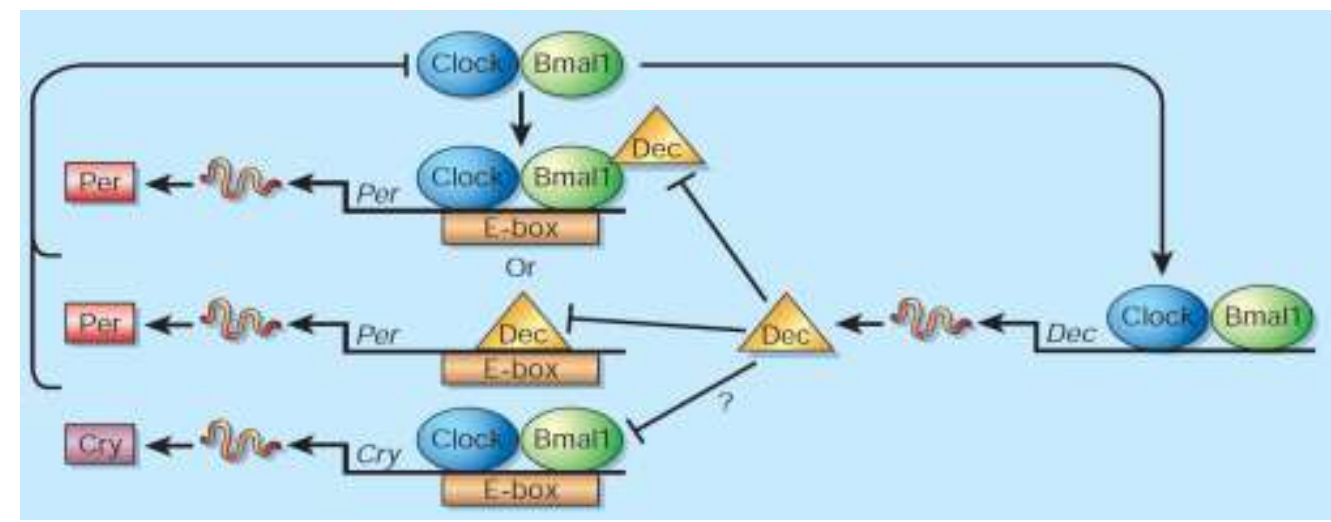

Figure 8: DEC regulation. Transcription of Dec genes is activated by CLOCK/BMAL1 heterodimer complexes. The DEC proteins repress Per transcription by interfering with CLOCK/BMAL1 activity or by binding to E-box. (Alvarez and Sehgal 2002)

\subsubsection{Peripheral clocks}

The circadian clock is not only present in the SCN, but also in peripheral cells. Microarray studies identified oscillating genes in many mammalian organs and tissues, e.g. liver, skeletal muscle and brown and white adipose tissues (Akhtar, Reddy et al. 2002; Kita, Shiozawa et al. 2002; Panda, Antoch et al. 2002; Storch, Lipan et al. 2002; Ueda, Chen et al. 2002; Reddy, Karp et al. 2006; Zvonic, Ptitsyn et al. 2006; McCarthy, Andrews et al. 2007), suggesting that the circadian system influences biosynthetic and metabolic processes such as cholesterol and lipid metabolism, glycolysis and gluconeogenesis, oxidative phosphorylation and detoxification pathways. The molecular clock of peripheral cells is based on a phase-delayed $(4-12 \mathrm{~h}$ relative to the circadian oscillation in the SCN) TTL involving the same or homolog clock components. Cultured 
cells and tissue explants from liver, lung, kidney, spleen etc. exhibit robust circadian oscillations in gene expression and Per2::Luc explants from SCN and tissues of the periphery show also robust rhythms (Balsalobre, Damiola et al. 1998; Yamazaki, Numano et al. 2000; Yoo, Yamazaki et al. 2004; Yamazaki, Yoshikawa et al. 2009). This indicates a hierarchical timekeeping system where time information is sent from the SCN to semi-autonomous oscillators in the periphery to synchronize the physiology of peripheral organs. Each individual cell possesses its own self-sustained circadian oscillator and the damping clock rhythms observed in cell culture (e.g. fibroblasts) are due to desynchronization between individual oscillators, rather than due to the loss of rhythmicity in individual cells (Nagoshi, Saini et al. 2004; Welsh, Yoo et al. 2004) (Figure 9).
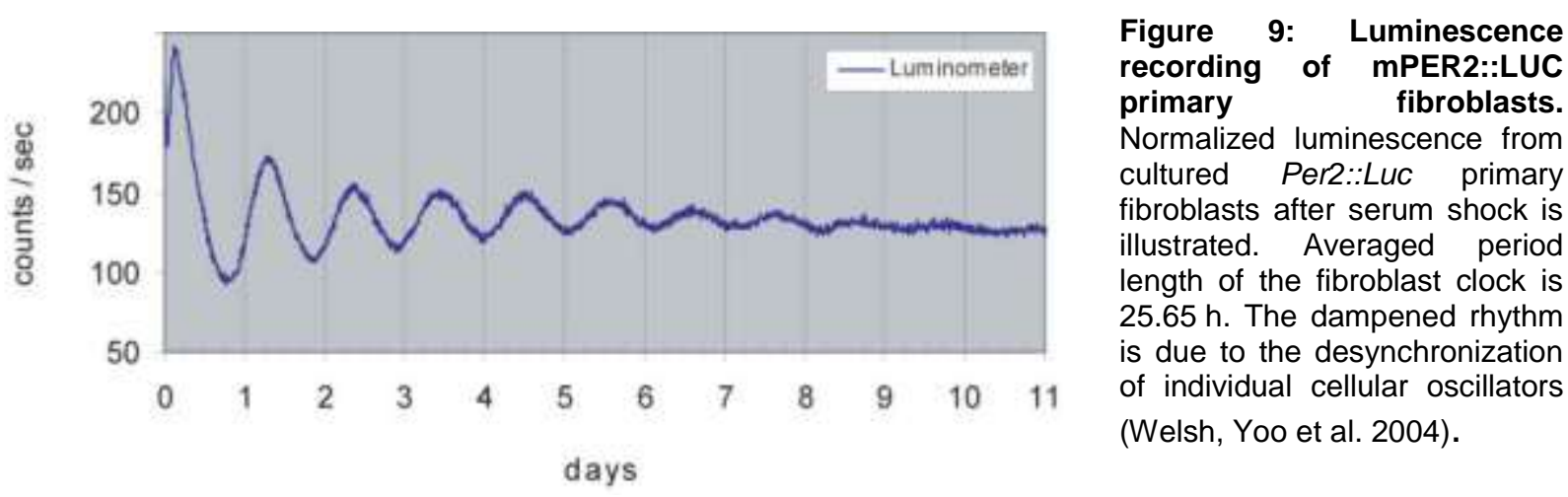

\subsection{Clock mutants}

To understand the role of each clock gene, mutant or knock out animals of clock genes have been generated and investigated. $C_{l o c k}{ }^{\Delta 19 / \Delta 19}$ mutant mice - the Clock ${ }^{\Delta 19}$ mutant allele encodes a dominant negative version of CLOCK that binds BMAL1, but lacks transcriptional activity - display a lengthened period and become arrhythmic in constant darkness (Vitaterna, King et al. 1994). In contrast, the phenotype of Clock $^{-/}$mice is milder (Debruyne, Noton et al. 2006) which might be explained by a certain redundancy with the CLOCK paralog NPAS2 (Reick, Garcia et al. 2001; DeBruyne, Weaver et al. 2007). Bmal1 $1^{-}$mice show impaired entrainment to LD cycles and, upon release into constant darkness, behavioural and molecular rhythms are lost indicating a complete disruption of the circadian clock (Bunger, Wilsbacher et al. 2000). These observations suggest that CLOCK/NPAS2 and BMAL1 are critical components of the molecular circadian clock. 
The clock continues to oscillate in Per1, Per2, Cry1 or Cry2 single mutant mice (van der Horst, Muijtjens et al. 1999; Vitaterna, Selby et al. 1999; Zheng, Larkin et al. 1999; Bae, Jin et al. 2001; Cermakian, Monaco et al. 2001; Zheng, Albrecht et al. 2001). The DD period length of Per1 and Per2 mutant mice is shortened compared to wild-type mice and Per2 mice gradually become arrhythmic under these conditions (Zheng, Larkin et al. 1999; Zheng, Albrecht et al. 2001). Cry $1^{-/}$mice have a shorter and $\mathrm{Cry}^{-/}$mice a longer period length in constant darkness compared to wild-type animals (van der Horst, Muijtjens et al. 1999; Vitaterna, Selby et al. 1999). The simultaneous disruption of Per1 and Per2 or of Cry 1 and Cry2 causes behavioural and molecular arrhythmicity (van der Horst, Muijtjens et al. 1999; Vitaterna, Selby et al. 1999; Bae, Jin et al. 2001; Zheng, Albrecht et al. 2001) highlighting an essential role for PER and CRY in the circadian system. Per2/Cry1 double mutants show disrupted activity and clock gene expression rhythms in the SCN and several tissues. In contrast, Per2/Cry2 mutant mice display normal circadian rhythmicity indicating that PER1/CRY1, but not PER1/CRY2, complexes are sufficient to drive the circadian clock (Oster, Yasui et al. 2002). Additionally Per1/Cry1 and Per1/Cry2 mutant mice display rhythmic activity and circadian clock gene expression, but Per1/Cry2 animals loose rhythmicity in an age-dependent manner. Thus, the $\mathrm{PER} / \mathrm{CRY}$ complexes have different potentials to regulate the circadian clock and not all interactions between PER and CRY are equal in vivo (Oster, Yasui et al. 2002; Oster, Baeriswyl et al. 2003). Nevertheless, PER and CRY are necessary for a functional clock.

Dec1 (Bhlhe40 ${ }^{\text {tm1Tan })}$ and Dec2 (Bh/he41 ${ }^{\text {tm1Miro }}$ ) mutants entrain readily to LD cycles and have stable rhythms under constant darkness conditions with normal period lengths (Dec single mutants) or with a lengthened period (Dec1/2 double mutants) compared to wild-type mice (Rossner, Oster et al. 2008). In contrast, a study by Nakashima and colleagues showed a lengthened DD period for Dec $1^{-/}$mice (Nakashima, Kawamoto et al. 2008). Photic phase resetting is preserved in Dec single mutants, but in the absence of both $D e c$ genes clock resetting is impaired with a reduced phase delay response (Rossner, Oster et al. 2008). In a jet lag paradigm, Dec1 single and Dec1/2 double mutants re-entrain slower to delayed LD cycles while Dec single mutant mice re-entrain faster to an advanced LD cycle (Nakashima, Kawamoto et al. 2008; Rossner, Oster et al. 2008). Gene expression of Per1,2 and Bmal1 in the cerebral cortex and the liver is elevated in Dec1/2 mutant mice, but Bmal1 mRNA accumulation is unchanged and Per2 transcription is blunted in the SCN of these animals. In contrast, Dec single mutants show normal Per2 and Bmal1 transcription in the SCN (Rossner, Oster et al. 2008). 


\subsection{Photic resetting of the circadian system}

In a natural environment, circadian rhythms in animals have to be frequently reset to stay entrained to the day-night cycle because the endogenous clock period is not exactly $24 \mathrm{~h}$. Photic resetting can be studied by short exposure to light during the night, which resets circadian activity rhythms. Pittendrigh showed that early-night light exposure delays the circadian clock, such that the animal awakens later (Pittendrigh 1976). A light pulse during late night, on the other hand, advances the circadian clock and the animal awakens earlier. An overview of the daily variation in the ability of the murine clock to shift its phase in response to photic stimuli can be represented by a phase response curve (PRC) as shown in Figure 10. Light exposure does not affect the circadian phase when applied during the subjective day (dead zone).

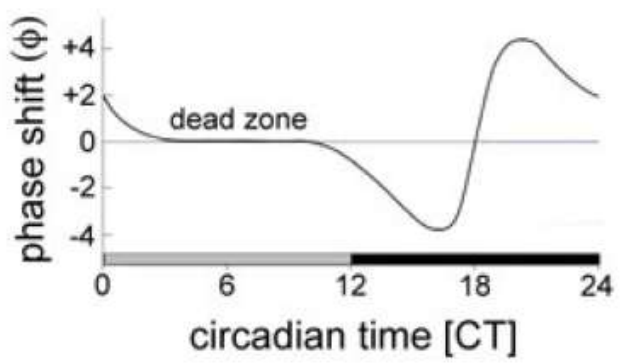

Figure 10: Photic phase response curve (PRC). The PRC was determined using an Aschoff type I protocol. Light pulse exposure between CT11 and CT18 induces phase delays (negative values). Light pulses between CT19 and CT3 generate phase advance responses (positive values). Between CT4 and CT10 no phase shift can be observed (dead zone). The grey and black bars represent subjective day and night, respectively. Modified from (Jud, Schmutz et al. 2005)

To reset the circadian clock, light must alter the phase of the TTL in the SCN. Photic phase resetting of the SCN clock is believed to depend on acute photic Per1 and Per2 (and possibly Dec1) induction in SCN neurons after light exposure during the night (Albrecht, Sun et al. 1997; Shearman, Zylka et al. 1997; Shigeyoshi, Taguchi et al. 1997; Miyake, Sumi et al. 2000; Honma, Kawamoto et al. 2002; Yan and Silver 2002; Rossner, Oster et al. 2008). Via photic activation of Per expression, PER protein levels increase in the SCN neurons with a delay (Yan and Silver 2004). This may shift the phase of the TTL, although the mechanism is still unknown. In line, Per2 and Per1 single mutant mice show decreased behavioural responses to nocturnal light exposure in the first or second half of the dark phase, respectively (Albrecht, Zheng et al. 2001; Spoelstra, Albrecht et al. 2004). Dec1/2 double mutants show reduced resetting which is restricted to phase delays (Rossner, Oster et al. 2008).

Of note, other resetting mechanisms have been reported at the posttranscriptional level. Photic activation of, and consecutive interaction with protein kinase $C$ alpha (PKCa) 
causes a temporary stabilization and cytoplasmatic retention of PER2 protein. This leads to a prolongation of the PER/CRY negative feedback on CLOCK/BMAL1, and a phase delay of the TTL (Jakubcakova, Oster et al. 2007).

\subsection{Clinical aspects}

In mammals, the circadian clock controls a large number of physiological variables. Therefore, it is not surprising that circadian disruption is associated with various diseases and physiological disorders. Sleep disorder is a medical disorder of sleep patterns including delayed sleep phase syndrome (DSPS) and advanced sleep phase syndrome (ASPS). DSPS patients prefer to go to bed very late at night and sleep late in the morning, resulting in a shift of peak alertness to midnight. In ASPS, the opposite situation occurs. DSPS and ASPS can be hereditary. In one form of familial advanced sleep phase syndrome (FASPS), a mutation in the human PER2 gene was identified leading to decreased PER2 phosphorylation by CK1 and reduced protein turnover (Jones, Campbell et al. 1999; Toh, Jones et al. 2001).

Restless legs syndrome (RLS) is a neurological sleep disorder characterized by an urge to move the limbs, accompanied by periodic limb movements (PLM) during sleep, leading to severe insomnia (Walters 1995; Allen, Picchietti et al. 2003). The symptoms are generally present during periods of rest and more frequent in the evening or night. In RLS circadian rhythms appear not to be altered, as shown by normal $24 \mathrm{~h}$ profiles of physiological markers such as core body temperature, cortisol and melatonin secretion (Wetter, Collado-Seidel et al. 2002; Tribl, Waldhauser et al. 2003; Michaud, Dumont et al. 2004). However, because of the diurnality of RLS symptoms an implication of the circadian clock in RLS etiology has been proposed. Michaud et al. suggest that melatonin might be involved in the worsening of RLS symptoms in the evening and at night. Further, a therapeutic effect of melatonin in patients with PLM but without RLS has been reported (Kunz and Bes 2001). The pathophysiological basis of RLS remains unclear, but pharmacological data indicate the dopaminergic system plays an essential role (Hening, Allen et al. 1999; Kraus, Schuld et al. 1999).

Jet lag is a consequence of desynchrony between the circadian system and external time as a result of crossing several time zones. Depending on the extent of the phase shift, the circadian system requires several days to fully adapt to the destination time zone (Srinivasan, Singh et al. ; Kiessling, Eichele et al. 2010). The adaptation after westbound 
flights is faster than after eastbound flights due to the fact that the clock has a better capacity for phase delays than phase advances (see also PRC, Figure 10) (Khalsa, Jewett et al. 2003). Jet lag affects sleep-wake cycles as well peripheral physiology, e.g. in the gastrointestinal tract, liver, pancreas and kidney (Waterhouse, Reilly et al. 2007).

A circadian mood disorder is seasonal affective disorder (SAD) - also called winter depression. SAD is characterized by recurrent depression that occurs annually, usually at the same time each year, for several years (Rosenthal, Sack et al. 1984). SAD is predominantly found in northern countries during wintertime correlating with very short daylight periods. Lewy et al. postulated a phase shift hypothesis of SAD which suggests that seasonal depression occurs when intrinsic circadian rhythms, such as melatonin and temperature rhythms, are phase delayed relative to external time and/or the sleep/wake cycle (Lewy and Sack 1988). This phase shift can be alleviated by light therapy (exposure to artificial bright light) (Lewy, Kern et al. 1982; Rosenthal, Sack et al. 1984; Wirz-Justice, Bucheli et al. 1986) and/or administration of melatonin.

It was suggested that the circadian system also plays a role in mental disorders such as major depressive disorder (MDD; unipolar disorder) and bipolar disorder (BPD). Circadian symptoms of MDD include insomnia, elevated core body temperature (Persaud 2000) and cortisol (Gold, Drevets et al. 2002; Keller, Flores et al. 2006) and lower melatonin secretion (Paparrigopoulos 2002). BPD is frequently associated with insomnia or hypersomnia, early-morning awakenings, reduced sleep efficiency and reduced sleep latency. Phase advances in the diurnal rhythms of plasma cortisol (Linkowski, Mendlewicz et al. 1985) implicate circadian disturbances in BPD. Clock mutant mice show a manialike phenotype with increased reward values of appetitive stimuli and reduced depressive and anxiety-like behavior (Roybal, Theobold et al. 2007).

Circadian parameters are disrupted in a wide spectrum of further central nervous system (CNS) disorders such as Parkinson's disease, Alzheimer's disease and dementia (Barnard and Nolan 2008). It is, however, difficult to define whether the disruption in circadian parameters is just a consequence of CNS disease, or whether it contributes to the development of the disorder.

A whole range of metabolic diseases are associated with misalignment of the circadian clock and metabolism (Kovac, Husse et al. 2009). Shift workers possess an elevated risk for the development of obesity, type II diabetes, metabolic syndrome and cardiovascular complications (Bray and Young 2007). Animal models further support a crucial role for the circadian clock in the regulation of metabolism. Clock mutant mice show increased food 
intake and develop obesity and a diabetic phenotype (Turek, Joshu et al. 2005). In another mouse model of obesity (KK-A $\mathrm{A}^{\mathrm{y}}$ mice) clock gene rhythms in liver and white adipose tissues are severely attenuated (Ando, Yanagihara et al. 2005).

Circadian clocks were shown to be involved in cell cycle regulation and thus might play a role in cancer development. Clock gene mutations (e.g. in Per genes) increase the incidence of cancer. Strongly dysregulated PER expression is found in Taiwanese woman with breast cancer (Chen, Choo et al. 2005) and Per2 mutant mice spontaneously develop tumours, e.g. lymphomas (Fu, Pelicano et al. 2002; Lee 2006). It was shown that light exposure at night might promote cancer development (Travis, Allen et al. 2004; Schernhammer, Berrino et al. 2008), increasing the risk of colorectal cancer in night-shift workers (Schernhammer, Laden et al. 2003).

\subsection{Aim}

Synergistic function of the circadian transcription factors PER and CWO was postulated for the Drosophila clock (Kadener, Stoleru et al. 2007). Based on the high conservation between the fruit fly and the mammalian circadian system, we hypothesized a similar synergism between $\operatorname{Per}(1,2)$ and $\operatorname{Dec}(1,2)$ in the murine circadian clock.

In vitro, it is very difficult to mimic spatial and temporal clock depending interactions. Some of the proposed components of the mammalian oscillators are important in vitro, but appear not relevant for clock function in vivo such as Per3 (Shearman, Jin et al. 2000). Therefore, we chose to study the $\operatorname{Per}(1,2)-D e c$ interaction in the living animal. We generated homozygous $\operatorname{Per} 1 / \operatorname{Dec}(1,2)$ or $\operatorname{Per} 2 / \operatorname{Dec}(1,2)$ double mutant mice by crossing Per and Dec mutant animals. It was postulated that Dec1 and Dec2 have redundant functions (Rossner, Oster et al. 2008). Therefore, we also generated and investigated Per/Dec triple mutant mice. These mutants allowed us to assess Per and Dec functional interaction in behaviour using locomotor activity measurements under different conditions, i.e. under entrained conditions and free-run, to analyze the endogenous Per-Dec interaction in the circadian system in vivo.

In vitro, the transcription factors DEC1 and DEC2 were shown to act as bidirectional transcriptional regulators, i.e. co-activators and repressors (Rossner, Oster et al. 2008). Thus, the in vivo regulatory function of the Per-Dec interactions in the circadian pacemaker, the SCN, was also studied in these mutants. 


\section{Chapter 2: Material and Methods}

\subsection{Animals}

Per2 $^{m / m}$ (Per2 ${ }^{\text {Brdm1 }}$; Zheng, Larkin et al. 1999), Per1 ${ }^{-/-}$(Per1 $^{\text {Brdm1 }}$; Zheng, Albrecht et al.

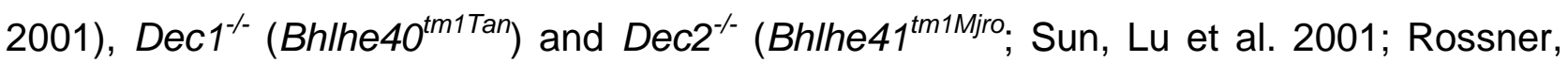
Oster et al. 2008) mice were backcrossed to a C57BL/6J background (N10). Homozygous single mutants were then mated to obtain homozygous $\mathrm{Per1}^{-/} \operatorname{Dec} 1^{-/}$, Per1 ${ }^{-/} \operatorname{Dec} 2^{-/}$, Per2 ${ }^{m / m} \operatorname{Dec1}^{-/}$and Per2 ${ }^{m / m} \operatorname{Dec}^{-/-}$double mutants as well Per1 ${ }^{-/}$Dec $1 / 2^{-/-}$and Per2 ${ }^{m / m}$ Dec $1 / 2^{-/-}$triple mutant mice. Figure 11 shows the breeding strategy for the generation of these mutants. The genotypes of the offspring were determined by PCR as described previously (Zheng, Larkin et al. 1999; Zheng, Albrecht et al. 2001; Rossner, Oster et al. 2008). The behavioural paradigms were performed on the homozygous double and triple mutants.

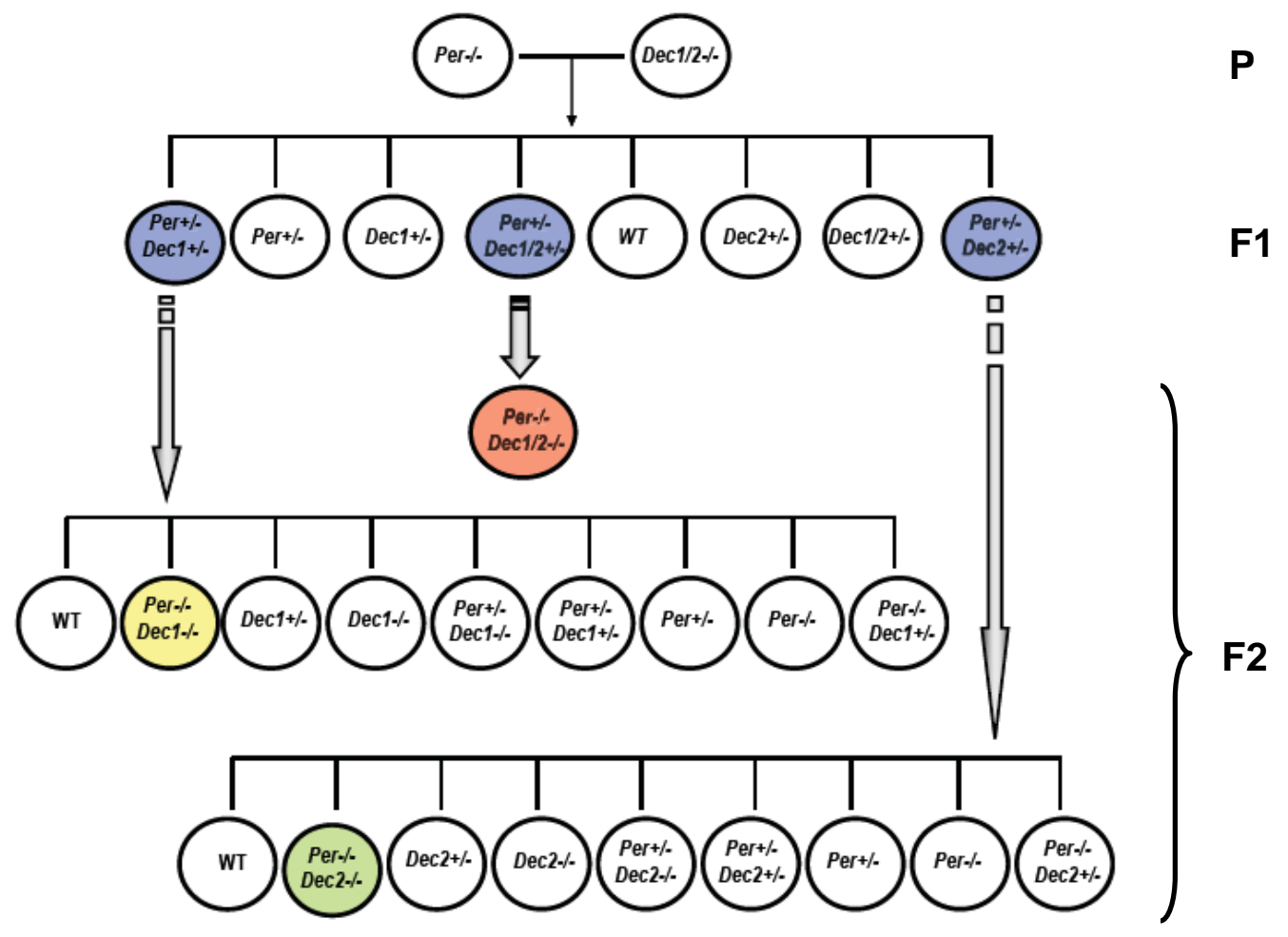

Figure 11. Breeding scheme for the generation of Per/Dec double and triple mutant mice. Bl6/J-backcrossed homozygous Per1 or Per2 single mutant mice were mated with Bl6/J-backcrossed homozygous Dec single mutant mice to generate within two generations (F2) homozygous Per/Dec1 (yellow), Per/Dec2 (green) double and Per/Dec1/2 (red) triple mutant mice. 


\subsection{Behavioural paradigms}

\subsubsection{Running-wheel assays}

Locomotor activity was analyzed using running wheels. All experiments were performed on male adult animals (2-10 months) and congenic C57BI/6J mice were used as controls. Figure 12 shows a comparable installation of the isolated cabinets used, holding 12 wheel-running cages. The light conditions were regulated by computercontrolled fluorescent lights in the ceiling of each cabinet: light intensity variations between cages were less than \pm 10 lux at 250 lux. Temperature was $20^{\circ} \mathrm{C} \pm 0.5^{\circ} \mathrm{C}$ and humidity was $60 \% \pm 5 \%$ throughout all experiments. Each cabinet was individually ventilated at $20 \mathrm{x}$ vol. per hour. Under normal conditions, the light was set to a $12 \mathrm{~h}$ light : 12 hours dark (LD) cycle with a light intensity of 250 lux during the light phase. Mice were individually housed in running wheel-equipped cages (Figure 12A) with water and food ad libitum. Running-wheel activity was individually recorded using ClockLab software on a computer running Windows XP.

A
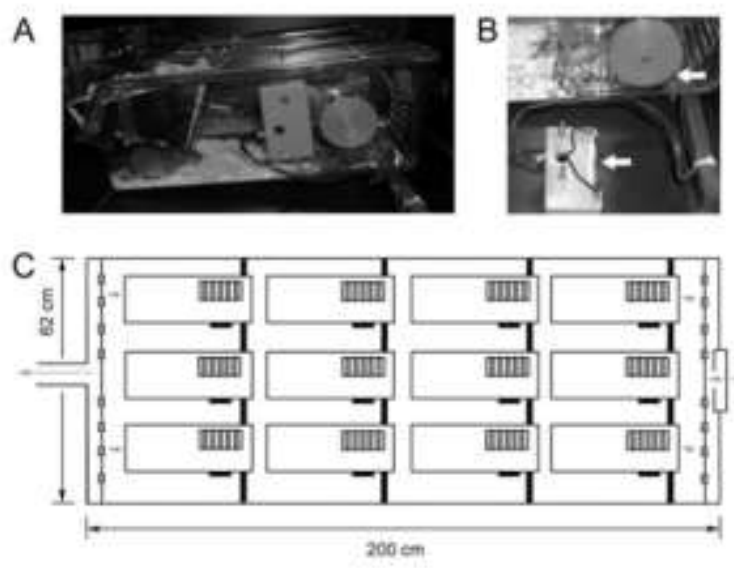

D

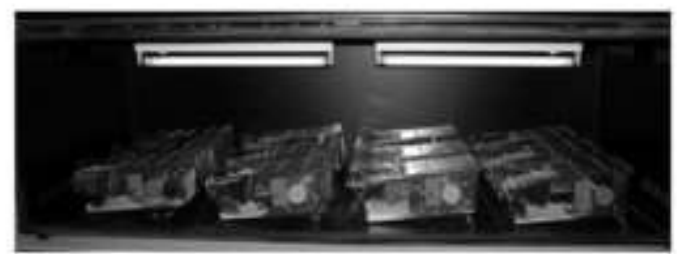

Figure 12. Overview of the cabinet with 12 wheel-running cages. (A) Wheel-running cage with an individually housed mouse. The running-wheel is connected via a magnetic switch for recording. With each rotation of the running wheel, the magnetic switch is opened and closed once. (B) The magnet (upper arrow) rotates simultaneously with wheel-revolution and the magnetic switch (lower arrow) transmits the wheel-revolutions to the computer. (C) Schematic representation of a cabinet with 12 wheel-running cages. (D) Picture of cabinet with two light bulbs (our setup held 4). From (Jud, Schmutz et al. 2005) 


\subsubsection{Masking}

To test for photic masking capacities, animals were entrained to a $12 \mathrm{~h}: 12 \mathrm{~h}$ LD cycle for 10 days. On day 10, a light pulse of 100 lux was applied between ZT14 and ZT15. Before light administration, chow was removed from the hopper at ZT12 and some chow pellets were put directly into the cage to ensure comparable light exposures. Figure 13A depicts an exemplary actogram of wild-type mice during experimental days 7-10. Masking capacity was calculated as the running-wheel activity during the light exposure period (ZT14-15) on day 10 divided by the average running-wheel activity without light treatment on days 7-9 for the same animal at ZT14-15 (represented in Figure 13B as light phase).

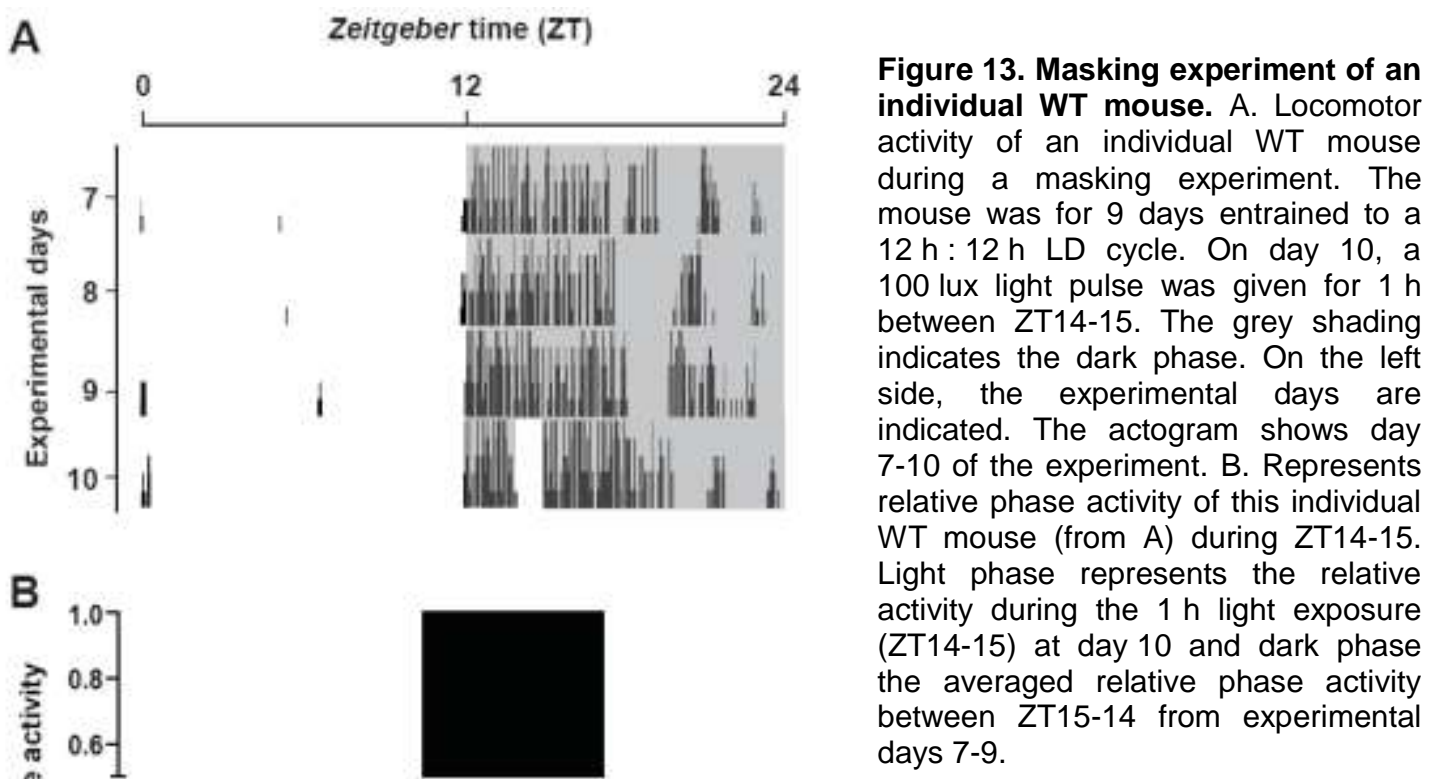

\subsubsection{Phase delay resetting}

Activity phase delay shifting by single nocturnal light pulses (LP) was performed using an Aschoff type II protocol (LD $\rightarrow \mathrm{LP} \rightarrow \mathrm{DD}$ ) because Per2/Dec mutant animals become arrhythmic under constant darkness which prevents stable determination of activity onsets over extended times in DD. All animals were entrained to a $12 \mathrm{~h}: 12 \mathrm{~h}$ LD cycle with a 
light intensity of 250 lux for two weeks. A 15 min light pulse (250 lux light intensity) was applied during the first half of the dark phase (ZT15) before animals were released into DD. Alternatively the LP was applied on the first day in DD at CT14. The phase shift response of each animal was determined as the difference between the regression lines through onsets before and after the light pulse on the first day after the light exposure (Figure 14 lower panel). This was compared to animals of the same genotype, which were released into DD without prior light exposure (Figure 14 upper panel) to extract the phase shift which was mediated by the light exposure from that produced by the mere release into DD.

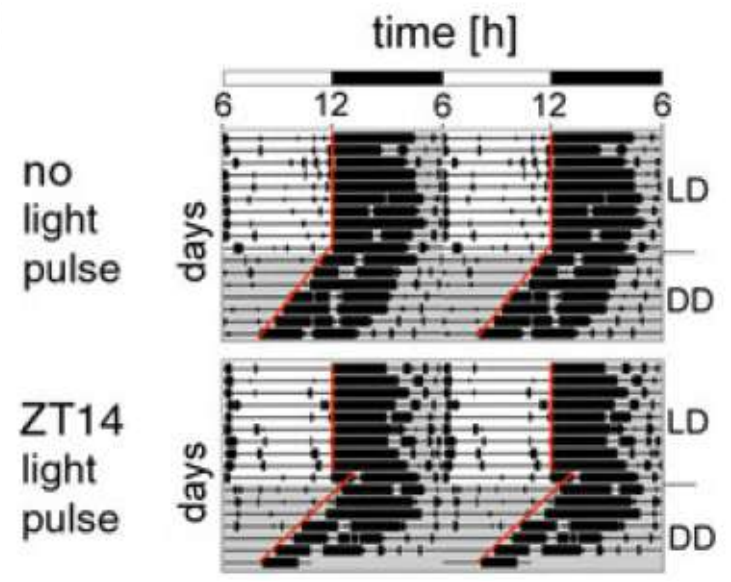

Figure 14. Typical actograms of WT animals in an Aschoff type II resetting protocol. Upper panel shows no light pulse treatment. Mice were entrained to a $12 \mathrm{~h}: 12 \mathrm{~h}$ LD cycle for 10 days and then released into DD. Lower panel shows a light pulse application for $15 \mathrm{~min}$ to the entrained mouse at ZT14. The phase shift is calculated by comparing the regression lines (red line) drawn through the onsets of wheel-running activity on the last days in LD and the first days in DD. The grey background represents darkness. Modified from (Jud, Schmutz et al. 2005) 


\section{Chapter 3: Results}

3.1. 'Genetic interaction of Per1 and Dec1/2 in the regulation of circadian locomotor activity' (submitted to Journal of Biological Rhythms)

Brid Bode, Ali Shahmoradi, Moritz J. Rossner, Henrik Oster 


\begin{abstract}
In mammals circadian rhythms of behavior and physiology are controlled by a hierarchical system of endogenous clocks, with a circadian pacemaker located in the suprachiasmatic nuclei (SCN) of the hypothalamus that synchronizes peripheral oscillators found in most other tissues. The molecular clock machinery is regulated by interlocked transcriptional translational feedback loops (TTLs). The mammalian core TTL includes the transcriptional modulators PER (1-3) and CRY (1/2) that feedback on their own expression by interaction with CLOCK/BMAL1. An accessory loop involving the transcription factors DEC1 and DEC2 has been described that also impinges on CLOCK/BMAL1-mediated transactivation. In Drosophila the DEC ortholog CWO shows synergistic activity to PER. This prompted us to analyze PER1-DEC interaction in the mammalian SCN pacemaker. We generated Perl/Dec double and triple mutant mice to monitor circadian wheel-running behavior under entrained and free-running conditions. Further, we analyzed circadian expression profiles of the core clock genes Per2, Rev-Erb $\alpha$ and Bmall in wild-type and Per1/Dec mutant SCN by in situ hybridization. The behavioral experiments revealed a critical role for Perl-Dec interaction in the synchronization of activity phase under entrained conditions. In constant darkness a synergistic function for Perl and Decl/2 in period regulation was found, correlating with disrupted clock gene transcription rhythms in the SCN. Together, our results suggest a partially redundant and bidirectional regulatory function for the two Dec genes in the TTL and a conservation of $\mathrm{Per}-\mathrm{Dec}(\mathrm{Cwo})$ synergism between vertebrate and invertebrate clocks.
\end{abstract}

Key words: Perl, Dec1, Dec2, photic entrainment, free-run, SCN, mice 


\section{INTRODUCTION}

In most species $24 \mathrm{~h}$ rhythms of physiology and activity are regulated by endogenous circadian clocks. In mammals behavioral rhythms are controlled by a circadian pacemaker located in the suprachiasmatic nuclei (SCN) of the hypothalamus (Moore and Eichler 1972; Schibler and Sassone-Corsi 2002; Stephan and Zucker 1972). The SCN shows electrical activity rhythms of only approximately $24 \mathrm{~h}$ under free-running conditions (Inouye and Kawamura 1979). Therefore, to keep in synchrony with external time, the internal clock has to be reset each day by environmental Zeitgebers in a process termed entrainment. The predominant Zeitgeber of the mammalian system is light which reaches the SCN via the retino-hypothalamic tract (Moore and Lenn 1972). From the SCN, time information is transferred by various means to peripheral circadian oscillators found in all tissues and controlling local physiological rhythms (Buijs and Kalsbeek 2001; Oster 2006; Reppert and Weaver 2002).

At the molecular level SCN and peripheral clocks are based on interlocked transcriptional/translational feedback loops (TTLs) comprised from a set of clock genes that are highly preserved between vertebrate and invertebrate species (Zhang and Kay 2010). In the mammalian core TTL the transcription factors circadian locomotor output cycles kaput (CLOCK) and brain and muscle ARNT-like 1 (BMAL1, ARNTL) heterodimerize and activate the transcription of cis-regulatory E-box containing target genes including Period (Per1-3) and Cryptochrome (Cry1-2). PER/CRY protein complexes accumulate during the course of the day and in the night relocate back into the nucleus where they inhibit their own transcription by interaction with CLOCK/BMAL1 (Griffin et al. 1999; Kume et al. 1999; Reppert and Weaver 2002). Recently two other transcription factors, DEC1 (BHLHE40) and DEC2 (BHLHE41), have been described to interact with the components of the circadian core TTL in the SCN (Honma et al. 2002; Noshiro et al. 2005). Dec mutant mice show moderate defects in light entrainment and free-running periodicity (Rossner et al. 2008). Dec1 and Dec2 transcription is activated by CLOCK/BMAL1 and nocturnal light exposure acutely stimulates Decl expression in the SCN (Butler et al. 2004; Honma et al. 2002). On the other hand DEC1 and DEC2 impinge on circadian transcription by competing with CLOCK/BMAL1 for E-box occupation and/or by direct physical interaction with BMAL1 (Honma et al. 2002; Sato et al. 2004). The mode of interaction between the DECs and the other CLOCK/BMAL1 regulators - PERs and CRYs - however, remains unclear. Recent results suggest that DEC function can be either activatory or inhibitory, depending on tissue type as well as on promoter of target genes (Azmi et al. 2003; Dardente et al. 2009; Hamaguchi et al. 2004; Honma et al. 2002; Kawamoto et al. 2004; Li et al. 2004; Li et al. 2003; Rossner et al. 2008). 
Studies in flies have proposed that clockwork orange (cwo), the Drosophila ortholog of the Decs, has a synergistic function to the Perl/2 ortholog period (per) (Kadener et al. 2007). To test if this mode of interaction is preserved in mammals we analyzed circadian activity rhythms and clock gene expression in the SCN of Perl/Dec double and triple deficient mice. Our results show a synergistic function of Perl and Decl together with Decl/Dec2 redundancy in the regulation of photic activity entrainment and of period length under free-running conditions. Thus, it appears that per-cwo interaction in the Drosophila TTL is largely conserved in the mammalian pacemaker. 


\section{MATERIALS AND METHODS}

\section{Animals}

Perl mutant mice (Perl ${ }^{\text {Brdml }}$; Zheng et al. 2001) were back-crossed to a C57BL/6J background (N10) and mated with $D e c 1^{-/}$and $D e c 2^{-/}$mice (Rossner et al. 2008) to produce double mutant $\mathrm{Perl}^{-/} \mathrm{Decl}^{-/-}$and $\mathrm{Perl}^{-/-} \mathrm{Dec} 2^{-/}$as well as triple mutant $\mathrm{Perl}^{-/} \mathrm{Decl} / 2^{-/-}$mice. The genotype of the offspring was determined by PCR as described (Rossner et al. 2008; Zheng et al. 2001). All experiments were done on male adult animals (2-10 months). Congenic age-matched C57B1/6J mice were used as controls. All animal experiments were performed with permission from the Office of Consumer Protection and Food Safety of the State of Lower Saxony and in accordance with the German Animal Welfare Act.

\section{Behavioral analysis}

Mouse housing and handling were performed as described (Albrecht and Oster 2001). Wheelrunning activity was analyzed using ClockLab software (Actimetrics, Evanston, IL). Prior to the experiments, animals were entrained to a 12 hours light: 12 hours dark (LD) cycle for at least 10 days (light intensity 250 lux). For analyzing free-running locomotor activity under constant darkness conditions (DD), lights were turned off at the end of the light phase (Zeitgeber time ZT12) and not turned on again on the next day. Activity profiles in LD, DD and constant light (LL, light intensity 50 lux) were assessed over a time of 4-7 consecutive days. The onset phase angle was determined by fitting a straight line to 7-10 consecutive activity onsets using ClockLab software and manual correction. Onset variation depicts the mean deviation of real activity onsets from a regression line through 7-10 consecutive onsets under stably entrained or free-running conditions. Rhythmicity and period length $(\tau)$ in DD and LL were determined by $\mathrm{X}^{2}$ periodogram analysis over an interval of 7-10 consecutive days. Period differences were calculated by comparison of period lengths between two different DD intervals separated by at least 10 days in LD.

\section{In situ hybridization}

Animals were entrained to LD for 10 days, released into DD and sacrificed on the second day in DD at $6 \mathrm{~h}$ intervals. Brains were dissected under dim red light, fixed by immersion in $4 \% \mathrm{PFA}$ 
in PBS, dehydrated and paraffin embedded. $8 \mu \mathrm{m}$ sections were hybridized with ${ }^{35} \mathrm{~S}$-UTP-labeled antisense RNA probes for clock gene transcripts as described (Oster et al. 2003; Oster et al. 2002). The Rev-Erba probe template was generated by PCR (forward primer: CCCTCTACAGTGACAGCTCCA, reverse primer: TCAGACACCGTTTGTACTGGA) from murine adipose tissue cDNA. Relative quantification of expression levels was performed by densitometric analysis of autoradiograph films using Quantity One software (Bio-Rad, Munich, Germany). Background correction was performed by subtracting the optical density measured in the lateral hypothalamus. For each experiment 3 animals per genotype were used and 3 adjacent SCN sections per animal were analyzed.

\section{Reporter gene assays}

Dual reporter gene assays in HEK293 cells were performed as previously described (Rossner et al. 2008; Wehr et al. 2006) using a firefly luciferase Bmall reporter gene (Bmall::luc; Nagoshi et al. 2004) and a Renilla luciferase reporter as normalizing transfection control. CMV-driven expression plasmids coding for HA-tagged rat DEC1, Flag-tagged mouse DEC2 and V5-tagged mouse PER1 were used (Rossner et al. 2008). All firefly luciferase data were normalized to the Renilla luciferase data for the same sample. DEC1, DEC2 and PER1 encoding constructs were assembled using Gateway-mediated recombination as described (Wehr et al. 2006). The PER1 expression plasmids were kindly provided by Dr. Pablo Szendro, Max Planck Institute for Biophysical Chemistry, Göttingen, Germany.

\section{Statistical analysis}

Statistical evaluation was performed using GraphPad Prism software (GraphPad, La Jolla, CA). Groups were compared by One-way ANOVA followed by Tukey's posttest for multiple comparisons. A p-value $<0.05$ was considered significant. The rhythmicity of clock gene expression was evaluated using sine curve fitting with CircWave v1.4 (Oster et al. 2006). 


\section{RESULTS}

\section{Synergistic interaction of Per1 and Dec1/2 under entrained conditions}

Perl/Dec double and triple mutant mice were born at the expected Mendelian ratios - taking into account that both Dec genes are located relatively close together on the same chromosome (Fujimoto et al. 2001; Sun et al. 1999). Mutant mice of all genotypes were viable and fertile and did not show any gross anatomical or behavioral abnormalities (data not shown). To study PerlDec interaction in the regulation of circadian entrainment Perl/Dec double and triple mutant mice were housed individually and their running-wheel activity was monitored under a $12 \mathrm{~h}$ light: $12 \mathrm{~h}$ dark cycle (LD). Under these conditions all animals entrained readily to the light/dark cycle (Figure 1). However, significantly advanced activity onsets were observed in Perl single ($0.29 \pm 0.02 \mathrm{~h})$ as well as Perl/Decl $(-0.56 \pm 0.05 \mathrm{~h})$, Perl/Dec2 $(-0.28 \pm 0.05 \mathrm{~h})$ double and Perl/Dec1/2 $(-0.81 \pm 0.04 \mathrm{~h})$ triple mutant mice (Figure 2A and statistics in Table S1). $\mathrm{PerI}^{-/} \mathrm{Decl}^{-/-}$as well as $\mathrm{Perl}^{-/} \mathrm{Dec} 1 / 2^{-/-}$mice showed significantly earlier activity onsets in comparison to wild-type (WT) and to single Perl mutant animals. In addition, the activity onsets of Per1/Dec1/2 triple mutant mice were significantly advanced compared to those of both double mutant strains, indicating a synergistic interaction of Perl and Decl/2 and a certain functional redundancy of Dec1 and Dec2 in photic activity entrainment. These observations were supported when we measured entrainment stability by quantifying the day-to-day variation of activity onsets under LD conditions. Perl single as well as Perl/Dec double and triple mutant mice displayed decreased onset stability when compared to WT animals (Figure 2B). While single mutants showed onset variations between 0.11 and $0.19 \mathrm{~h}\left(\right.$ Perl $^{-/} 0.19 \pm 0.03 \mathrm{~h}, \mathrm{Dec}^{-/-} 0.11 \pm 0.02 \mathrm{~h}$ and Dec $2^{-/-}$ $0.14 \pm 0.02 \mathrm{~h}$ ), this value increased gradually with decreasing Perl/Dec gene dosage (Per ${ }^{-/}$Dec $2^{-/-}$ $0.26 \pm 0.03 \mathrm{~h}$, Perl $^{-/}$Decl $^{-/} 0.30 \pm 0.03 \mathrm{~h}$ and Perl ${ }^{-/}$Dec1/2 ${ }^{-/} 0.48 \pm 0.04 \mathrm{~h}$ ), suggesting that a fully functional Perl-Dec interaction is necessary for efficient synchronization of activity phase with the external LD cycle.

\section{Functional redundancy of Dec1 and Dec2 in period regulation and Perl interaction under free-running conditions}

We next asked whether the Perl-Dec synergism observed in LD is conserved under freerunning conditions, i.e. in the absence of external Zeitgeber signaling. To test this, we released the animals into constant darkness (DD). In DD, all mutants retained rhythmic circadian activity 
patterns (Figure 1), but marked changes in the period of these rhythms were observed. With the exception of $D e c 2^{-/}$mice $(23.56 \pm 0.03 \mathrm{~h})$, all single, double and triple mutant genotypes displayed a significantly shortened DD period length when compared to wild-type controls (Per ${ }^{-/}$ $22.39 \pm 0.07$ h, Decl $1^{-/} 23.07 \pm 0.06$ h, Perl ${ }^{-/}$Decl $^{-/} 22.28 \pm 0.10$ h, Perl $1^{-/} D_{e c 2^{-/}} 22.23 \pm 0.11$ h, Perl $^{-/}$Dec1/2/- $21.68 \pm 0.10$ h; WT $23.48 \pm 0.02$ h; Figure $2 \mathrm{C}$ and statistics in Table S1). While in the double mutant mice period length appeared mainly to be determined by the absence of Perl, Per1/Dec triple mutants showed a significant reduction in period length when compared to all single and double mutant strains. Similar, though statistically less powerful, effects were observed under constant light (LL) conditions. In LL, a loss of Perl led to an increase in period length by about $1 \mathrm{~h}$ (WT $25.12 \pm 0.08$ h, Perl ${ }^{-/} 26.09 \pm 0.12 \mathrm{~h}$; Figure $\mathrm{S} 1$ and statistics in Table S1). While no period change was observed in either Decl or Dec2 single mutants, a combination of Perl and Decl/2 deficiency further increased LL free-running period in a Perl/Dec gene dosage dependent manner $\left(\right.$ Decl $^{-/} 25.10 \pm 0.09$ h, Dec2 $2^{-/} 25.18 \pm 0.06$ h, Perl $1^{-/}$Dec $^{-/-} 25.54 \pm 0.14 \mathrm{~h}$, Perl $^{-/}$Dec $^{-/-}$ $26.34 \pm 0.10 \mathrm{~h}, \mathrm{Perl}^{-/} \mathrm{Decl} / 2^{-/} 26.66 \pm 0.40 \mathrm{~h}$; Figure S1 and statistics in Table S1). While the DD/LL data suggest a redundancy for Dec1 and Dec2 in the regulation of free-running period, functional interaction between Perl and Dec was only observed in the absence of both Dec genes.

A previous study had shown that Perl deficiency results in a marked instability of free-running period length over extended periods of time (Zheng et al. 2001). To test whether the observed Perl-Dec interaction in LD entrainment extends to this phenomenon we re-entrained all mice to LD and then re-released them into DD to compare activity period lengths of individual mice between both free-running intervals. In WT animals, period length was very stable with an average period difference between both DD episodes of less than $0.2 \mathrm{~h}$ (WT $0.11 \pm 0.02 \mathrm{~h}$; Figure 2D). As expected, increased period variations were observed in Perl mutant mice (Perl ${ }^{-/} 0.41 \pm 0.06 \mathrm{~h}$; Figure 2D and statistics in Table S1), while no significant changes in period variability were seen in Decl and Dec2 single mutant animals $\left(\right.$ Dec $^{-/} 0.27 \pm 0.05 \mathrm{~h}, \operatorname{Dec}^{-/} 0.16 \pm 0.04 \mathrm{~h}$; Figure 2D and statistics in Table S1). Though a trend towards a further increase in period destabilization was seen in double and triple Perl/Dec mutants $\left(\right.$ Perl $^{-/}$Decl $^{-/} 0.56 \pm 0.05 \mathrm{~h}, \quad$ Perl ${ }^{-/}$Dec $^{-/-}$ $0.57 \pm 0.11 \mathrm{~h}$, Perl $^{-/}$Dec $1 / 2^{-/} 0.58 \pm 0.04 \mathrm{~h}$; Figure 2D and statistics in Table S1), none of the data reached significance when compared to Perl single mutant mice, indicating that long-term period stability is predominantly dependent on Perl function. In summary, our free-running activity data suggest a dominant role for Perl in the regulation of period length and stability while, in contrast to LD, functional interaction between Perl and Decl/2 was only detectable under triple mutant conditions. 


\section{Synergistic effect of Per1 and Dec1/2 on Bmal1 expression in the SCN}

In situ hybridization analysis was performed to extend our investigations to the molecular level. Core clock gene expression profiles were determined on coronal SCN sections of animals sacrificed at four different time points on the second day in DD. For Per2, no significant changes in expression levels were found on a single Perl or Dec deficient background (Figure S2A). All genotypes showed rhythmic Per2 mRNA levels with peak expression during the late subjective day and comparable amplitudes (Figure 3A). This situation was preserved in Per1/Dec double mutants. In Per1/Dec1/2 triple mutant animals, Per2 expression was significantly reduced at CT14, reflecting an accelerated breakdown of Per2 transcripts during the early subjective night and correlating with the shortened activity period observed in these animals (Figure 2C).

The effect on Bmall expression was even stronger in these animals. While only minor changes in Bmall transcript levels were observed in Perl/Dec single and double mutants (Figure 3C and Figure S2B), Bmall mRNA rhythms were completely abolished in the SCN of Perl/Dec triple mutant animals. In wild-type, single and double mutant mice, Bmall showed moderate rhythmic expression with peak levels between CT14 and CT20. In contrast, triple mutant $\mathrm{Perl}^{-/} \mathrm{Decl} / 2^{-/}$ mice displayed medium level Bmall transcript concentrations throughout the whole day (red line in Figure 3C). Thus, deficiency of, either, Per1, Dec1/2 or a combination of Perl and one Dec gene is dispensable for rhythmic Bmall expression, but at least one set of Dec alleles seems necessary for normal Bmall transcriptional regulation in the SCN.

So far, it is thought that PER and DEC proteins affect transcriptional regulation of the circadian TTL mainly by interfering with CLOCK/BMAL1-activated transcription via E-box enhancer elements (Honma et al. 2002; Reppert and Weaver 2002; Sangoram et al. 1998; Sato et al. 2004; Shearman et al. 2000). To test if the observed strong interactive effect of Perl and Decl/2 on (nonE-box-regulated) Bmall transcription is mediated via an intermediate E-box-controlled gene/protein other than Per2/PER2 we examined the circadian expression of Rev-Erb $\alpha$ (Nrld1), a potent negative regulator of circadian Bmall transcription (Preitner et al. 2002). In wild-type animals Rev-Erba expression in the SCN showed robust rhythms with peak levels during the early subjective day (Figure 3B and Figure S2C). Interestingly, and unlike what had been observed for Per2 and Bmal1, Rev-Erba levels were markedly changed in Per1, Dec1 and Dec2 single mutant mice with elevated expression during the second half of the subjective day (CT8; Figure S2C). This effect was also seen in Perl/Dec double and triple mutant animals. In Perl ${ }^{-/}$Decl $1 / 2^{-/}$mice this increase at CT8 resulted in a shift in expression peak phase by $4 \mathrm{~h}$ from CT4 to CT8 
(Figure 3B). In addition, Rev-Erb $\alpha$ expression at CT20 was reduced, indicating an altered phaserelationship between Per2 and Rev-Erba expression rhythms in these mice.

To clarify the role of Perl and Decs in Bmall transcriptional regulation, we performed luciferase reporter gene assays in HEK293 cells. In reporter cells transfected with Perl plasmid alone no changes in luciferase activity were observed. Transfection of one Dec plasmid moderately but significantly increased luciferase activity by a factor of 2-4.5, with a higher induction capacity of Dec2 (Figure 3D and statistics in Table S2; Dardente et al. 2009). This induction was unchanged after co-transfection with Perl in the presence of one Dec. However, co-transfection of both Decs together with Perl resulted in a strong further increase of luciferase activity (Figure 3D and statistics in Table S2). Thus, both DECs, have an activatory role on Bmall expression in vitro that is further potentiated by addition of Perl.

Taken together, DD expression profiling of core clock genes in the SCN and reporter gene assays revealed synergistic effects of Perl and Dec1/2 on Per2, Rev-Erb $\alpha$ and, most pronounced, on Bmall expression together with full functional redundancy of Decl and Dec2 and correlating with behavioral changes observed under the same conditions. 


\section{DISCUSSION}

In this study, we show by behavioral and molecular investigation of clock function in Perl/Dec double and triple mutant mice that the previously postulated synergistic function (Kadener et al. 2007) of Per and Dec genes in the regulation of the invertebrate circadian core TTL is conserved in mammals. Our findings reveal a functional specificity in the interaction of Dec1 and Dec2 with Perl in photic entrainment. Synergistic interactions of Dec genes with Perl are critical for the normal synchronization of activity phase to the external LD cycle. Under free-running conditions Perl-Dec synergy is preserved, but Decl and Dec2 show full functional redundancy in the modulation of rhythm period. In contrast, the stability of rhythm sustainment under constant darkness appears to be exclusively dependent on Perl function.

It has previously been shown that Perl and Dec single mutant mice entrain to LD cycles without any significant alterations in wheel-running activity when compared to wild-type littermates (Nakashima et al. 2008; Rossner et al. 2008; Spoelstra et al. 2004; Zheng et al. 2001). However, Perl mutant mice show a tendency towards advanced activity onsets under these conditions (Spoelstra et al. 2004). In our experiments, single, double and triple Perl/Dec mutant mice entrained readily to an LD cycle, but Perl single and Perl/Dec double and triple mutants exhibited strongly advanced activity onsets (Figure 2A) or predark activity (Mrosovsky 2001). The mechanism behind this phenomenon is still largely unknown, but seems to involve a disruption of the negative masking capacity of light towards the end of the day (Mrosovsky 2001). This masking effect was also suggested to underlie the normal entrainment behavior of the short period Perl mutants in bright LD conditions (Zheng et al. 2001). The observed Dec dosage dependent cumulative advance of activity onsets in Perl/Decl and Perl/Decl/2 mutants indicates a complementary, but specific, role of both Dec genes in this process. Given the lack of significant changes in activity onsets in Dec single mutants (Figure 2A; Rossner et al. 2008) the functional interaction with Perl seems essential for a normal phase relationship between activity and the external LD cycle. In humans, equivalents of advanced activity onsets are observed in advanced sleep phase syndrome (ASPS) (Wulff et al. 2009). In ASPS patients, Perl gene polymorphisms are associated with extreme morning preference and abnormal sleep patterns (Carpen et al. 2006). Further, the ASPS phenotype is also associated with Per2 polymorphisms while alterations in Per3 are correlated to late chronotypes (Archer et al. 2003; Ebisawa et al. 2001; Viola et al. 2007), suggesting a high level of interaction of the three Pers in this process (Jones et al. 1999; Toh et al. 2001). In contrast, Per2 as well as Perl/2 mutant mice show a disruption of sleep consolidation (Kopp et al. 2002; Shiromani et al. 2004) while Perl $^{-/-}$mice exhibit no abnormal sleep phenotype 
(Kopp et al. 2002). In contrast, in our study Per1Dec1/2 mutant mice show strongly advanced activity onsets (Figure 2A), suggesting that Perl-Dec interaction might be essential for these effects. Mutations in the human Dec2 gene are associated with a short sleep phenotype. However, Dec2 deficient mice exhibit only minor changes in sleep architecture (He et al. 2009). Consistent with this, neither Decl nor Dec2 single mutant animals showed changes in their activity onsets (Figure 2A, Table S1). In general, sleep is regulated by interplay of homeostatic and circadian processes (Dijk and Czeisler 1995). Therefore, the observed predark activity could also result from alterations in the regulation of sleep drive. Our LD data suggest a strong interaction of Perl and Decl/2 in the regulation of rest/activity cycles under entrained conditions. It would, therefore, be interesting to test if comparable genetic interactions can be observed in human sleep phase pathologies.

In constant conditions all Perl/Dec mutant strains retained stable rhythmic activity patterns. DD period length was shortened in Decl and Perl single mutant mice (Rossner et al. 2008; Zheng et al. 2001). In Per1/Dec double mutants period length was comparable to that of Perl ${ }^{-/}$mice, while in mice deficient for Perl and both Dec genes dramatically shortened circadian period lengths were observed (Figure 2C, Table S1), suggesting a full functional redundancy for both Decs in interacting with Perl under free-run conditions. This is supported by the constant light data (Figure S1). Following Aschoff's rule (Aschoff 1960) period lengths were increased in all investigated genotypes (Figure S1). Of all single mutants this effect was most pronounced in $\mathrm{Perl}^{-}$ /- animals (Figure S1; Steinlechner et al. 2002). While functional Dec1/2 redundancy seems to mask Perl-Dec synergism in double mutant animals Perl/Decl/2 triple mutants showed a further increase in period length.

Our in situ hybridization data suggest that either Perl, Decl or Dec2 are dispensable for rhythmic Per2 expression in the SCN in constant darkness (Figure 3, Figure S2; Rossner et al. 2008; Zheng et al. 2001). A moderate reduction in Per2 mRNA was seen in Per1/Dec1/2 triple mutants at CT14 (Figure 3A) supporting a certain level of redundancy of Decl and Dec2 function in Per2 transcriptional regulation. Studies on hepatic clock function indicate that Per2 rhythms unlike most other core clock genes - are stabilized by systemic factors independent of local TTL function (Kornmann et al. 2007). In line with this, changes in gene activity were more pronounced for Rev-Erba and Bmall in the triple mutant animals. Rhythmic Bmall transcription in the SCN seems to critically depend on the presence of at least one functional Dec gene (Figure 3C). Of note, dampened clock gene expression rhythms have been observed in Decl/2 double mutants (Rossner et al. 2008). Our reporter gene assay data postulate a synergistic activatory transcriptional role of PER1 and DEC1/2 on Bmall transcription (Figure 3D). It remains to be shown if this activatory 
function is direct - of note, the mouse Bmall promoter contains three non-canonical E-boxes (nucleotides 7,082-7,087 bp, 7,674-7,679 bp and 7,711-7,716 bp of GenBank AB064982.1) - or if it depends on PER1/DEC-mediated activation/inhibition of other Bmall regulators. Together, these data postulate a critical role for Per1-Decl/2 interaction for Bmall rhythmicity. In contrast to Per2 and Bmall, the amplitude of Rev-Erba mRNA rhythms in Perl/Decl/2 triple mutants was increased, indicating a primarily inhibitory function for Perl and Dec on Rev-Erb $\alpha$ transcription. A similar mode of action has been postulated for Perl (Preitner et al. 2002). In contrast, during the second half of the subjective night, Rev-Erb $\alpha$ expression was suppressed in Perl/Decl/2 mutants indicating an activating Dec function at this time (Figure 3B). Such a time of day-dependent transactivation functionality of Per-Dec is supported by a number of in vitro studies showing that DECs have a bidirectional regulatory function on the transcription of E-box containing genes (Azmi et al. 2003; Dardente et al. 2009; Hamaguchi et al. 2004; Honma et al. 2002; Kawamoto et al. 2004; Li et al. 2003; Rossner et al. 2008).

Of note, the strengthened rhythm in Rev-Erb $\alpha$ expression in the triple mutants does not result in a rhythmic Bmall expression (Figure 3, Figure S2). A possible explanation for this effect would be that the altered phase-relationship between the positive Bmall regulator Per2 (Schmutz et al. 2009; Shearman et al. 2000) and the transcriptional repressor Rev-Erbo (Preitner et al. 2002) results in a blunting of Bmall transcription. However, further transcriptional modulators such as RORs or other uncharacterized proteins as well as post-translational processes could also be involved. It is likely that the disrupted Bmall transcription in Perl/Dec triple mutants results in a weakened oscillator which is reflected in the shortened activity period of these animals in DD (Figure 2C). A similar cumulative effect on free-running period length was observed in other double mutant lines such as Per1/Rev-Erba and Per2/Rev-Erba (Jud et al. 2010; Schmutz et al. 2009).

It was recently postulated that in Drosophila CWO functionally synergizes with PER and has both activatory and inhibitory functions on CLK/CYC-activated transcription (Kadener et al. 2007; Richier et al. 2008). In this study, we show an analog Per (1)-Dec interaction in the mammalian TTL. The molecular mechanism behind the bi-directionality of PER and DEC action on E-boxmediated and/or Bmall transcription remains to be solved, but seems to depend not only on daytime and tissue-type, but also on lighting conditions and the absence or presence of other TTL proteins such as BMAL1 (Rossner et al. 2008) and PER1. 


\section{ACKNOWLEDGMENTS}

We would like to thank Dr. Reshma Taneja for the generous gift of Decl mutant mice and Drs. Johanna Barclay and Lars Geffers for critical reading of the manuscript. This project was funded by a single project grant (OS 353/2-1) and an Emmy Noether Fellowship (H.O.) of the German Research Foundation (DFG). 


\section{REFERENCES}

Albrecht U and Oster H (2001) The circadian clock and behavior. Behavioral brain research 125:89-91.

Archer SN, Robilliard DL, Skene DJ, Smits M, Williams A, Arendt J and von Schantz M (2003) A length polymorphism in the circadian clock gene Per3 is linked to delayed sleep phase syndrome and extreme diurnal preference. Sleep 26:413-415.

Aschoff J (1960) Exogenous and endogenous components in circadian rhythms. Cold Spring Harbor symposia on quantitative biology 25:11-28.

Azmi S, Sun H, Ozog A and Taneja R (2003) mSharp-1/DEC2, a basic helix-loop-helix protein functions as a transcriptional repressor of E box activity and Stra13 expression. The Journal of biological chemistry 278:20098-20109.

Buijs RM and Kalsbeek A (2001) Hypothalamic integration of central and peripheral clocks. Nat Rev Neurosci 2:521-526.

Butler MP, Honma S, Fukumoto T, Kawamoto T, Fujimoto K, Noshiro M, Kato Y and Honma K (2004) Dec1 and Dec2 expression is disrupted in the suprachiasmatic nuclei of Clock mutant mice. J Biol Rhythms 19:126-134.

Carpen JD, von Schantz M, Smits M, Skene DJ and Archer SN (2006) A silent polymorphism in the PER1 gene associates with extreme diurnal preference in humans. Journal of human genetics 51:1122-1125.

Dardente H, Fustin JM and Hazlerigg DG (2009) Transcriptional feedback loops in the ovine circadian clock. Comparative biochemistry and physiology 153:391-398.

Dijk DJ and Czeisler CA (1995) Contribution of the circadian pacemaker and the sleep homeostat to sleep propensity, sleep structure, electroencephalographic slow waves, and sleep spindle activity in humans. J Neurosci 15:3526-3538.

Ebisawa T, Uchiyama M, Kajimura N, Mishima K, Kamei Y, Katoh M, Watanabe T, Sekimoto M, Shibui K, Kim K, Kudo Y, Ozeki Y, Sugishita M, Toyoshima R, Inoue Y, Yamada N, Nagase T, Ozaki N, Ohara O, Ishida N, Okawa M, Takahashi K and Yamauchi T (2001) Association of structural polymorphisms in the human period3 gene with delayed sleep phase syndrome. $E M B O$ reports 2:342-346.

Fujimoto K, Shen M, Noshiro M, Matsubara K, Shingu S, Honda K, Yoshida E, Suardita K, Matsuda Y and Kato Y (2001) Molecular cloning and characterization of DEC2, a new member of basic helix-loop-helix proteins. Biochemical and biophysical research communications 280:164171. 
Griffin EA, Jr., Staknis D and Weitz CJ (1999) Light-independent role of CRY1 and CRY2 in the mammalian circadian clock. Science 286:768-771.

Hamaguchi H, Fujimoto K, Kawamoto T, Noshiro M, Maemura K, Takeda N, Nagai R, Furukawa M, Honma S, Honma K, Kurihara H and Kato Y (2004) Expression of the gene for Dec2, a basic helix-loop-helix transcription factor, is regulated by a molecular clock system. Biochem J 382:4350 .

He Y, Jones CR, Fujiki N, Xu Y, Guo B, Holder JL, Jr., Rossner MJ, Nishino S and Fu YH (2009) The transcriptional repressor DEC2 regulates sleep length in mammals. Science (New York, NY 325:866-870.

Honma S, Kawamoto T, Takagi Y, Fujimoto K, Sato F, Noshiro M, Kato Y and Honma K (2002) Dec1 and Dec2 are regulators of the mammalian molecular clock. Nature 419:841-844.

Inouye ST and Kawamura H (1979) Persistence of circadian rhythmicity in a mammalian hypothalamic "island" containing the suprachiasmatic nucleus. Proceedings of the National Academy of Sciences of the United States of America 76:5962-5966.

Jones CR, Campbell SS, Zone SE, Cooper F, DeSano A, Murphy PJ, Jones B, Czajkowski L and Ptacek LJ (1999) Familial advanced sleep-phase syndrome: A short-period circadian rhythm variant in humans. Nature medicine 5:1062-1065.

Jud C, Hayoz A and Albrecht U (2010) High amplitude phase resetting in Rev-erbalpha/Perl double mutant mice. PLOS ONE 5.

Kadener S, Stoleru D, McDonald M, Nawathean P and Rosbash M (2007) Clockwork Orange is a transcriptional repressor and a new Drosophila circadian pacemaker component. Genes Dev 21:1675-1686.

Kawamoto T, Noshiro M, Sato F, Maemura K, Takeda N, Nagai R, Iwata T, Fujimoto K, Furukawa M, Miyazaki K, Honma S, Honma K and Kato Y (2004) A novel auto-feedback loop of Decl transcription involved in circadian rhythm regulation. Biochemical and biophysical research communications 313:117-124.

Kopp C, Albrecht U, Zheng B and Tobler I (2002) Homeostatic sleep regulation is preserved in mPer1 and mPer2 mutant mice. Eur J Neurosci 16:1099-1106.

Kornmann B, Schaad O, Bujard H, Takahashi JS and Schibler U (2007) System-driven and oscillator-dependent circadian transcription in mice with a conditionally active liver clock. PLoS biology $5: \mathrm{e} 34$.

Kume K, Zylka MJ, Sriram S, Shearman LP, Weaver DR, Jin X, Maywood ES, Hastings MH and Reppert SM (1999) mCRY1 and mCRY2 are essential components of the negative limb of the circadian clock feedback loop. Cell 98:193-205. 
Li Y, Song X, Ma Y, Liu J, Yang D and Yan B (2004) DNA binding, but not interaction with Bmal1, is responsible for DEC1-mediated transcription regulation of the circadian gene mPer1. Biochem J 382:895-904.

Li Y, Xie M, Song X, Gragen S, Sachdeva K, Wan Y and Yan B (2003) DEC1 negatively regulates the expression of DEC2 through binding to the E-box in the proximal promoter. The Journal of biological chemistry 278:16899-16907.

Moore RY and Eichler VB (1972) Loss of a circadian adrenal corticosterone rhythm following suprachiasmatic lesions in the rat. Brain research 42:201-206.

Moore RY and Lenn NJ (1972) A retinohypothalamic projection in the rat. The Journal of comparative neurology 146:1-14.

Mrosovsky N (2001) Further characterization of the phenotype of mCry1/mCry2-deficient mice. Chronobiology international 18:613-625.

Nagoshi E, Saini C, Bauer C, Laroche T, Naef F and Schibler U (2004) Circadian gene expression in individual fibroblasts: cell-autonomous and self-sustained oscillators pass time to daughter cells. Cell 119:693-705.

Nakashima A, Kawamoto T, Honda KK, Ueshima T, Noshiro M, Iwata T, Fujimoto K, Kubo H, Honma S, Yorioka N, Kohno N and Kato Y (2008) DEC1 modulates the circadian phase of clock gene expression. Molecular and cellular biology 28:4080-4092.

Noshiro M, Furukawa M, Honma S, Kawamoto T, Hamada T, Honma K and Kato Y (2005) Tissue-specific disruption of rhythmic expression of Dec1 and Dec2 in clock mutant mice. J Biol Rhythms 20:404-418.

Oster H (2006) The genetic basis of circadian behavior. Genes, brain, and behavior 5 Suppl 2:7379.

Oster H, Damerow S, Hut RA and Eichele G (2006) Transcriptional profiling in the adrenal gland reveals circadian regulation of hormone biosynthesis genes and nucleosome assembly genes. J Biol Rhythms 21:350-361.

Oster H, Werner C, Magnone MC, Mayser H, Feil R, Seeliger MW, Hofmann F and Albrecht U (2003) cGMP-dependent protein kinase II modulates mPer1 and mPer2 gene induction and influences phase shifts of the circadian clock. Curr Biol 13:725-733.

Oster H, Yasui A, van der Horst GT and Albrecht U (2002) Disruption of mCry2 restores circadian rhythmicity in mPer2 mutant mice. Genes Dev 16:2633-2638.

Preitner N, Damiola F, Lopez-Molina L, Zakany J, Duboule D, Albrecht U and Schibler U (2002) The orphan nuclear receptor REV-ERBalpha controls circadian transcription within the positive limb of the mammalian circadian oscillator. Cell 110:251-260. 
Reppert SM and Weaver DR (2002) Coordination of circadian timing in mammals. Nature 418:935-941.

Richier B, Michard-Vanhee C, Lamouroux A, Papin C and Rouyer F (2008) The clockwork orange Drosophila protein functions as both an activator and a repressor of clock gene expression. $J$ Biol Rhythms 23:103-116.

Rossner MJ, Oster H, Wichert SP, Reinecke L, Wehr MC, Reinecke J, Eichele G, Taneja R and Nave KA (2008) Disturbed clockwork resetting in Sharp-1 and Sharp-2 single and double mutant mice. PLoS One 3:e2762.

Sangoram AM, Saez L, Antoch MP, Gekakis N, Staknis D, Whiteley A, Fruechte EM, Vitaterna MH, Shimomura K, King DP, Young MW, Weitz CJ and Takahashi JS (1998) Mammalian circadian autoregulatory loop: a timeless ortholog and mPer1 interact and negatively regulate CLOCK-BMAL1-induced transcription. Neuron 21:1101-1113.

Sato F, Kawamoto T, Fujimoto K, Noshiro M, Honda KK, Honma S, Honma K and Kato Y (2004) Functional analysis of the basic helix-loop-helix transcription factor DEC1 in circadian regulation. Interaction with BMAL1. European journal of biochemistry / FEBS 271:4409-4419.

Schibler U and Sassone-Corsi P (2002) A web of circadian pacemakers. Cell 111:919-922.

Schmutz I, Ripperger JA, Baeriswyl-Aebischer S and Albrecht U (2009) The mammalian clock component PERIOD2 coordinates circadian output by interaction with nuclear receptors. Genes Dev 24:345-357.

Shearman LP, Sriram S, Weaver DR, Maywood ES, Chaves I, Zheng B, Kume K, Lee CC, van der Horst GT, Hastings MH and Reppert SM (2000) Interacting molecular loops in the mammalian circadian clock. Science 288:1013-1019.

Shiromani PJ, Xu M, Winston EM, Shiromani SN, Gerashchenko D and Weaver DR (2004) Sleep rhythmicity and homeostasis in mice with targeted disruption of mPeriod genes. Am J Physiol Regul Integr Comp Physiol 287:R47-57.

Spoelstra K, Albrecht U, van der Horst GT, Brauer V and Daan S (2004) Phase responses to light pulses in mice lacking functional per or cry genes. J Biol Rhythms 19:518-529.

Steinlechner S, Jacobmeier B, Scherbarth F, Dernbach H, Kruse F and Albrecht U (2002) Robust circadian rhythmicity of Per1 and Per2 mutant mice in constant light, and dynamics of Per1 and Per2 gene expression under long and short photoperiods. J Biol Rhythms 17:202-209.

Stephan FK and Zucker I (1972) Circadian rhythms in drinking behavior and locomotor activity of rats are eliminated by hypothalamic lesions. Proceedings of the National Academy of Sciences of the United States of America 69:1583-1586. 
Sun H, Mattei MG and Taneja R (1999) Assignment of stra13 to the sub-telomeric region of mouse chromosome 6 by in situ hybridization. Cytogenetics and cell genetics 87:211-212.

Toh KL, Jones CR, He Y, Eide EJ, Hinz WA, Virshup DM, Ptacek LJ and Fu YH (2001) An hPer2 phosphorylation site mutation in familial advanced sleep phase syndrome. Science 291:1040-1043. Viola AU, Archer SN, James LM, Groeger JA, Lo JC, Skene DJ, von Schantz M and Dijk DJ (2007) PER3 polymorphism predicts sleep structure and waking performance. Curr Biol 17:613618.

Wehr MC, Laage R, Bolz U, Fischer TM, Grunewald S, Scheek S, Bach A, Nave KA and Rossner MJ (2006) Monitoring regulated protein-protein interactions using split TEV. Nature methods 3:985-993.

Wulff K, Porcheret K, Cussans E and Foster RG (2009) Sleep and circadian rhythm disturbances: multiple genes and multiple phenotypes. Curr Opin Genet Dev 19:237-246.

Zhang EE and Kay SA (2010) Clocks not winding down: unraveling circadian networks. Nat Rev Mol Cell Biol 11:764-776.

Zheng B, Albrecht U, Kaasik K, Sage M, Lu W, Vaishnav S, Li Q, Sun ZS, Eichele G, Bradley A and Lee CC (2001) Non-redundant roles of the mPerl and mPer2 genes in the mammalian circadian clock. Cell 105:683-694. 


\section{FIGURE LEGENDS}

Figure 1. Wheel-running behavior under entrained and free-running conditions. (A-G) Representative locomotor activity records of (A) wild-type (WT), (B) $\mathrm{Perl}^{-/-}$, (C) Decl ${ }^{-/-}$, (D)

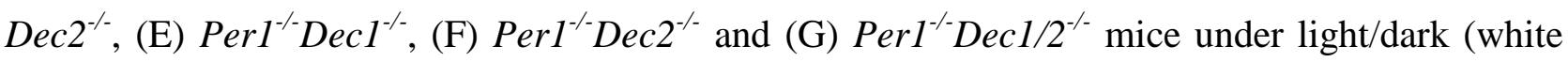
and grey shaded, respectively) and constant darkness (grey shaded) conditions. Black bars indicate wheel-revolutions per 6 min bin. Actograms are double-plotted with the activity of the following day plotted to the right and below the previous one. Gray shadings indicate dark phases.

Figure 2. Analysis of locomotor behavior under LD and DD conditions. (A) Activity onset phase angles relative to ZT12 ("lights off") under LD conditions: wild-type (WT) $-0.04 \pm 0.01 \mathrm{~h}(\mathrm{n}=20)$, Perl $^{-/}-0.29 \pm 0.02 \mathrm{~h} \quad(\mathrm{n}=20), \operatorname{Decl}^{-/}-0.19 \pm 0.02 \mathrm{~h} \quad(\mathrm{n}=20), \operatorname{Dec}^{-/-} \quad 0.01 \pm 0.02 \mathrm{~h} \quad(\mathrm{n}=12)$, Perl $^{\text {- Decl }}{ }^{-/-}-0.56 \pm 0.05 \mathrm{~h} \quad(\mathrm{n}=20)$, Perl $^{-/}$Dec $^{-/}-0.28 \pm 0.05 \mathrm{~h} \quad(\mathrm{n}=17)$ and Perl ${ }^{-/}$Decl/2 ${ }^{-/}$ $-0.81 \pm 0.04 \mathrm{~h}(\mathrm{n}=11)$. (B) Activity onset variations under LD conditions: WT $0.05 \pm 0.004 \mathrm{~h}$ $(\mathrm{n}=20)$, Perl $^{-/} 0.19 \pm 0.03 \mathrm{~h}(\mathrm{n}=20), \operatorname{Dec}^{-/} 0.11 \pm 0.02 \mathrm{~h}(\mathrm{n}=20), \operatorname{Dec}^{-/} 0.14 \pm 0.02 \mathrm{~h}(\mathrm{n}=12)$, Perl $^{-/}$Decl $^{-/} 0.30 \pm 0.03 \mathrm{~h} \quad(\mathrm{n}=19), \quad$ Perl $^{-/}$Dec $^{-/} 0.26 \pm 0.03 \mathrm{~h} \quad(\mathrm{n}=17)$ and Perl ${ }^{-/}$Decl/2 ${ }^{-/}$ $0.48 \pm 0.04 \mathrm{~h}(\mathrm{n}=12)$. (C) Average free-running period length in constant darkness (DD): WT $23.48 \pm 0.02 \mathrm{~h} \quad(\mathrm{n}=20), \operatorname{Perl}^{-/} 22.39 \pm 0.07 \mathrm{~h} \quad(\mathrm{n}=20), \operatorname{Decl}^{-/} 23.07 \pm 0.06 \mathrm{~h} \quad(\mathrm{n}=20), \operatorname{Dec}^{-/}$ 23.56 \pm 0.03 h (n=12), Perl $^{-/} \operatorname{Decl}^{-/} 22.28 \pm 0.10$ h (n=14), Perl ${ }^{-/} \operatorname{Dec}^{-/-} 22.23 \pm 0.11$ h $(\mathrm{n}=17)$, Perl $^{-/}$Dec1/2 ${ }^{-/} 21.68 \pm 0.10 \mathrm{~h}(\mathrm{n}=12)$. (D) Period variability between two DD intervals: WT $0.11 \pm 0.02 \mathrm{~h} \quad(\mathrm{n}=10), \quad$ Perl $^{-/} \quad 0.41 \pm 0.06 \mathrm{~h} \quad(\mathrm{n}=10), \quad \operatorname{Decl}^{-/} \quad 0.27 \pm 0.05 \mathrm{~h} \quad(\mathrm{n}=10), \quad \operatorname{Dec} 2^{-/}$ $0.16 \pm 0.04 \mathrm{~h} \quad(\mathrm{n}=10), \operatorname{Perl}^{-/} \operatorname{Decl}^{-/} 0.56 \pm 0.05 \mathrm{~h} \quad(\mathrm{n}=10), \operatorname{Perl}^{-/} \operatorname{Dec}^{-/-} 0.57 \pm 0.11 \mathrm{~h} \quad(\mathrm{n}=10)$, Perl $^{-/}$Decl $1 / 2^{-/} 0.58 \pm 0.04 \mathrm{~h}(\mathrm{n}=12)$. Data are represented as mean \pm SEM. *: $\mathrm{p}<0.05$ compared

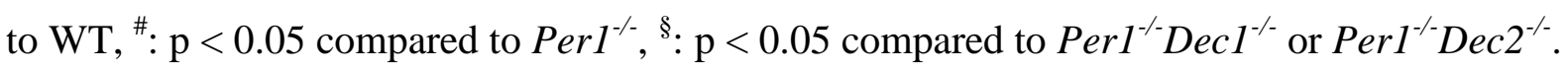

Figure 3. SCN clock gene expression and PER1/DEC influence on Bmall transcription. DD expression profiles of (A) Per2, (B) Rev-Erbo and (C) Bmall mRNA in the SCN of wild-type

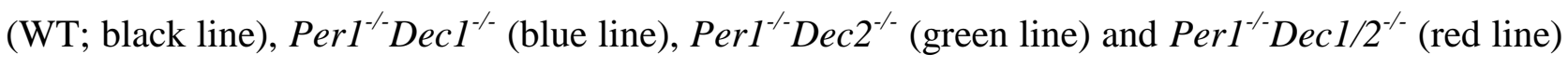
mice determined by $\mathrm{x}$-ray autoradiography of ${ }^{35} \mathrm{~S}$-labeled in situ hybridizations (ISH). Data are normalized to the average expression in wild-types and double plotted for visual clarity. All values are means \pm SEM $(n=3)$. (D) Dual luciferase reporter gene assays using a firefly Bmall::luc reporter construct, a Renilla luciferase reporter as transfection control and Dec1, Dec2 and/or Per1 expression plasmids in HEK293 cells. Data are normalized to Bmal1::luc control and represented as mean \pm SEM ( $\mathrm{n}=12$ replicates). $*: \mathrm{p}<0.05$ compared to Baml1::luc reporter (first column) or 
Bmall::luc reporter and Perl expression plasmid (second column), ${ }^{\S}: \mathrm{p}<0.05$ compared to Bmall::luc reporter, Perl and one Dec expression plasmid $\left(5^{\text {th }}\right.$ and $6^{\text {th }}$ column). RLU: relative luminescence units.

Figure S1. Locomotor activity in LL. Average free-running period length in constant light $\left(\mathrm{LL}_{50}\right.$ lux): WT 25.12 $\pm 0.08 \mathrm{~h}(\mathrm{n}=12), \operatorname{Perl}^{-/} 26.09 \pm 0.12 \mathrm{~h} \quad(\mathrm{n}=15), \operatorname{Decl}^{-/} 25.10 \pm 0.09 \mathrm{~h}(\mathrm{n}=11)$, $\operatorname{Dec}^{-/} 25.18 \pm 0.06 \mathrm{~h}(\mathrm{n}=12), \operatorname{Perl}^{-/} \operatorname{Decl}^{-/} 26.54 \pm 0.14 \mathrm{~h} \quad(\mathrm{n}=11), \operatorname{Perl}^{-/} \operatorname{Dec}^{-/} 26.34 \pm 0.10 \mathrm{~h}$ $(\mathrm{n}=11)$, Perl $^{-/} \operatorname{Decl}^{-/-} 26.92 \pm 0.22 \mathrm{~h}(\mathrm{n}=11)$. Data are represented as mean \pm SEM. * $: \mathrm{p}<0.05$ compared to WT, \#: $\mathrm{p}<0.05$ compared to Perl ${ }^{-/}$, ${ }^{\S}: \mathrm{p}<0.05$ compared to Perl ${ }^{-/}$Decl ${ }^{-/}$or $\operatorname{Per1}^{-/} \operatorname{Dec}^{-/}$.

Figure S2. In situ hybridization (ISH) profiles of clock gene expression in the SCN. Normalized expression of (A) Per2, (B) Bmall and (C) Rev-Erba mRNA in the SCN in DD of wild-type (WT; black line), $\operatorname{Perl}^{-/}$(red line), Decl ${ }^{-/}$(blue line) and $\operatorname{Dec}^{-/}$(green line) mice. Data are normalized to the average expression in wild-types and double plotted for visual clarity. All values are means $\pm \operatorname{SEM}(n=3)$. 

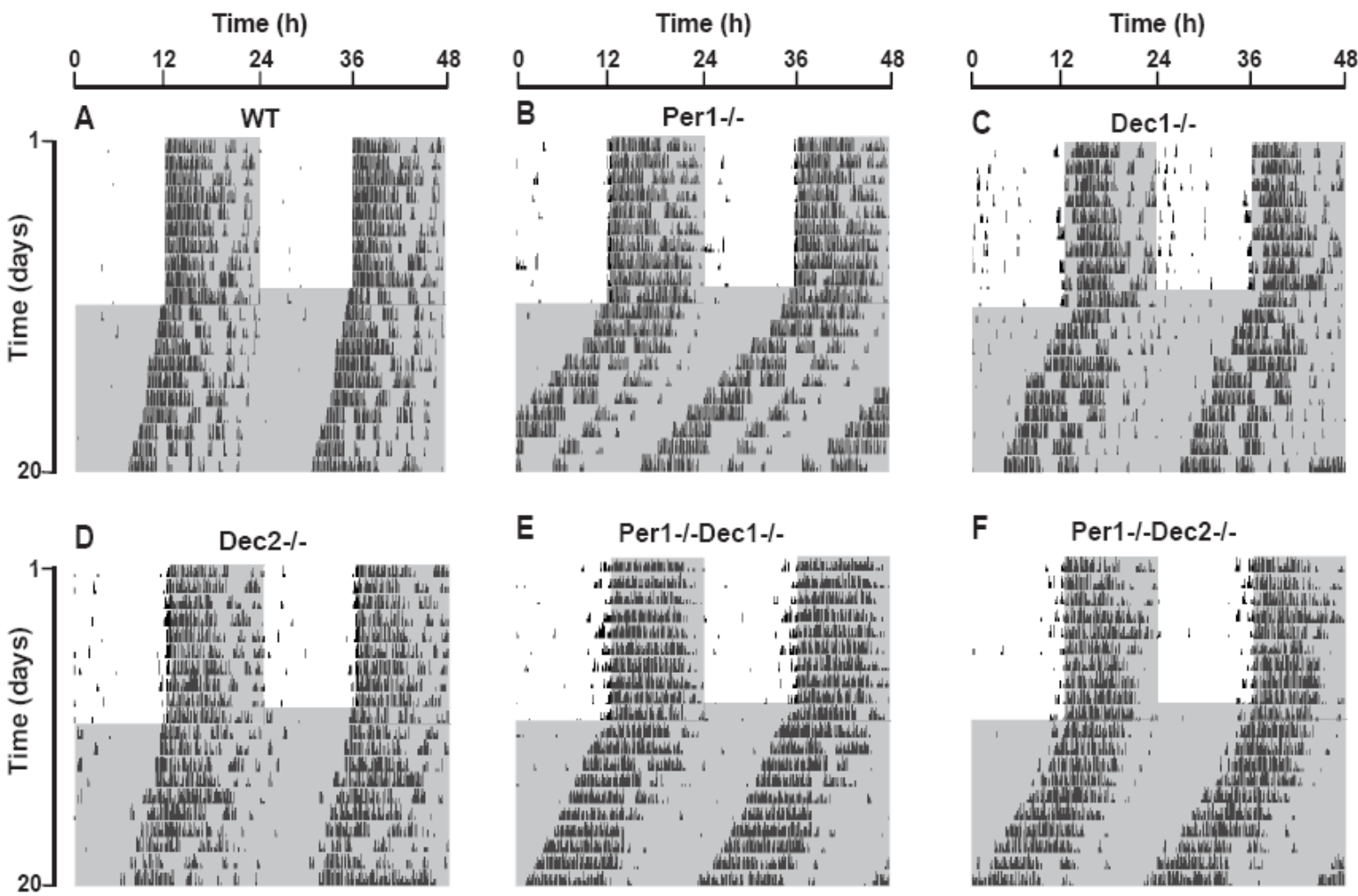

\section{E Per1-/-Dec1-/-}

F Per1-/-Dec2-/-
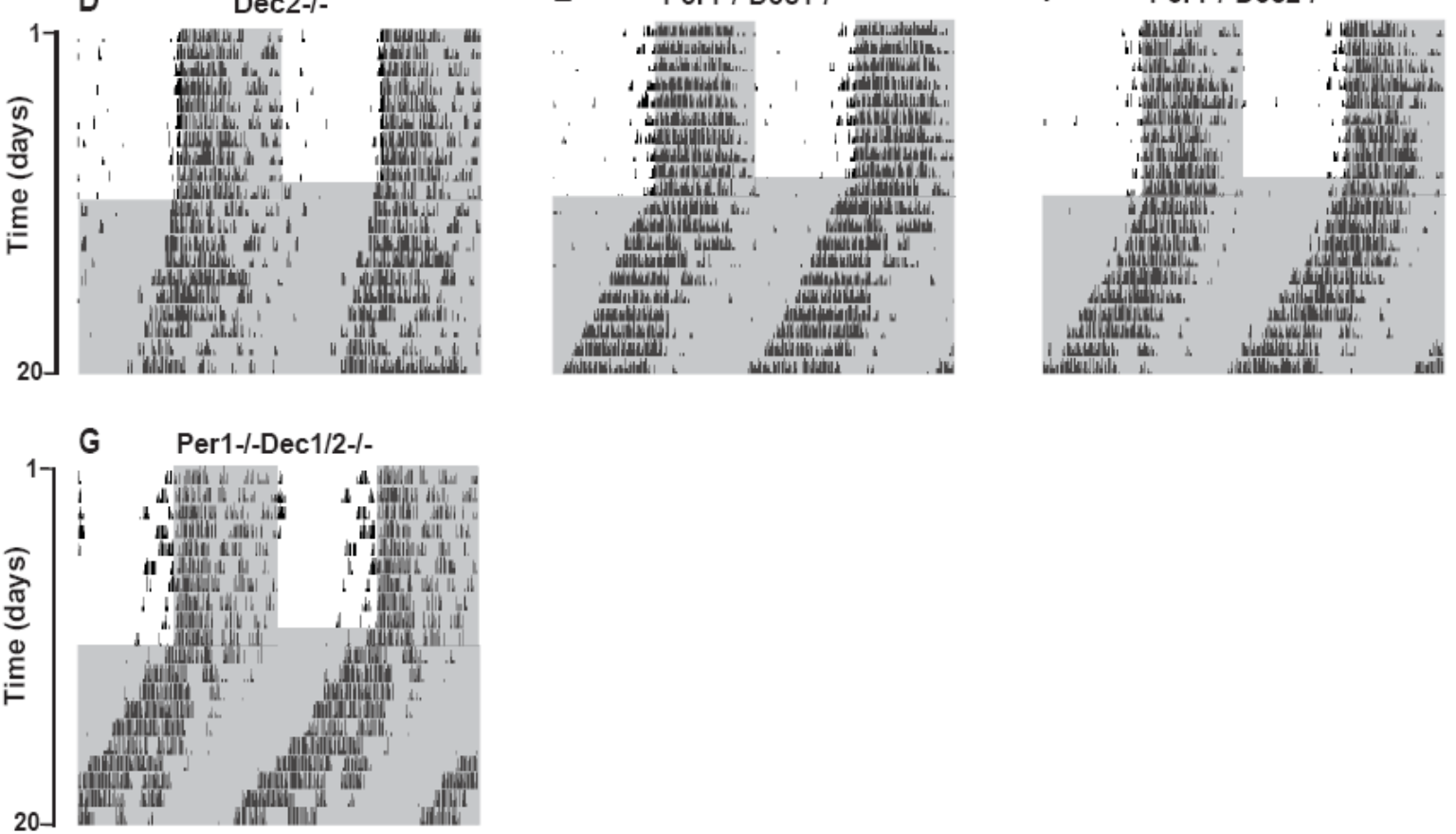
A

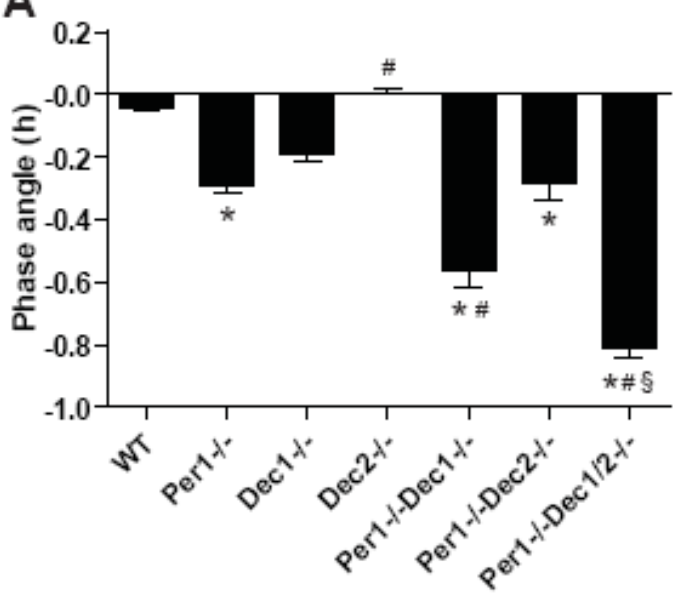

C

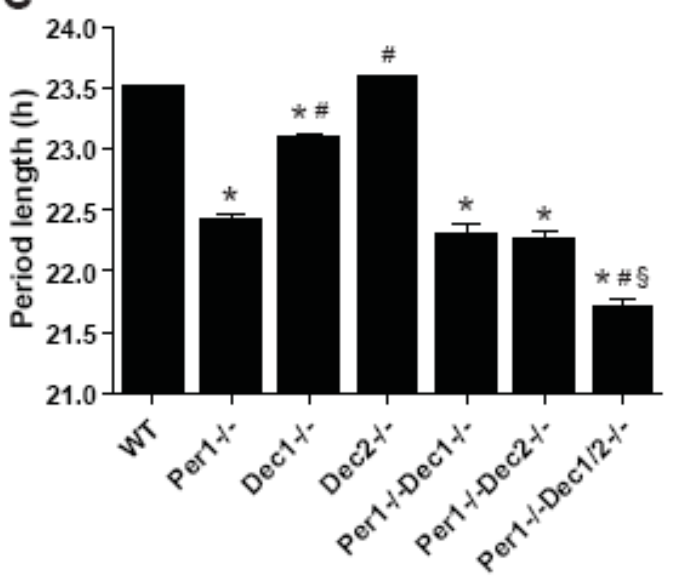

B

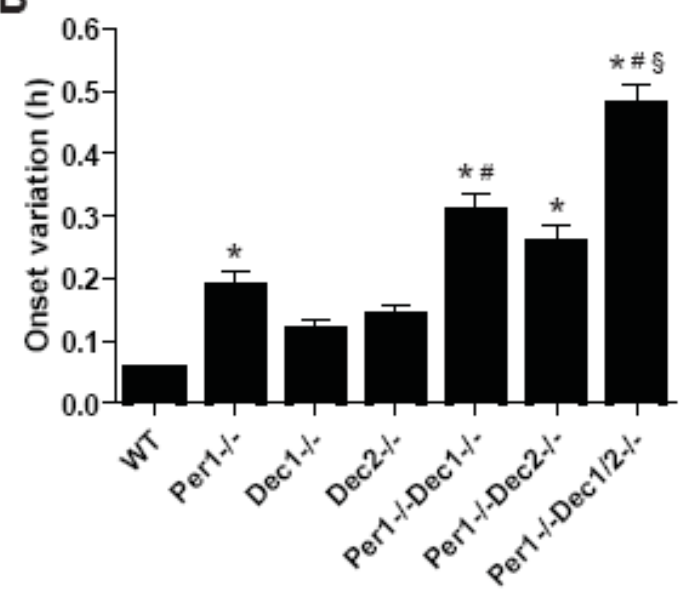

D

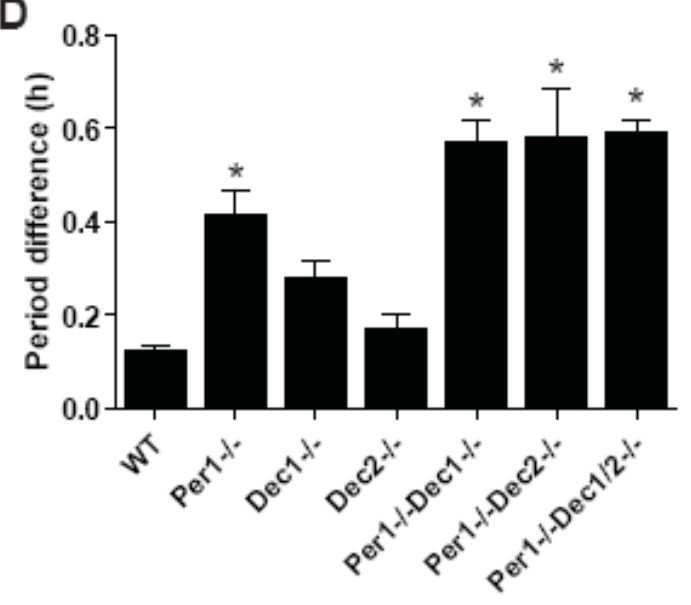


$\multimap$ WT $\rightarrow$ Per1-/-Dec1-/-
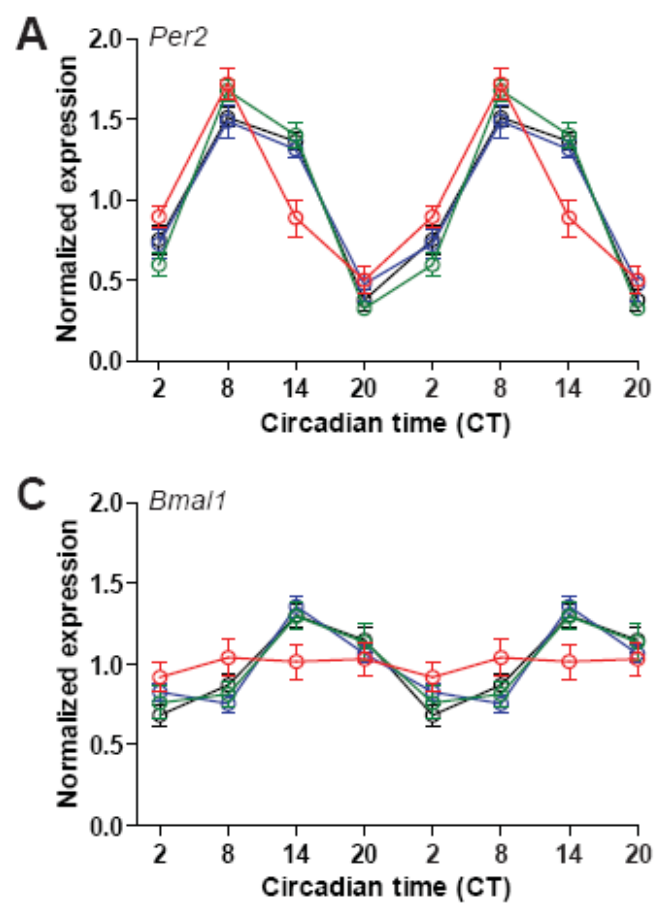

$\rightarrow$ Per1-/-Dec2-/- $\rightarrow$ Per1-/-Dec1/2-/

B
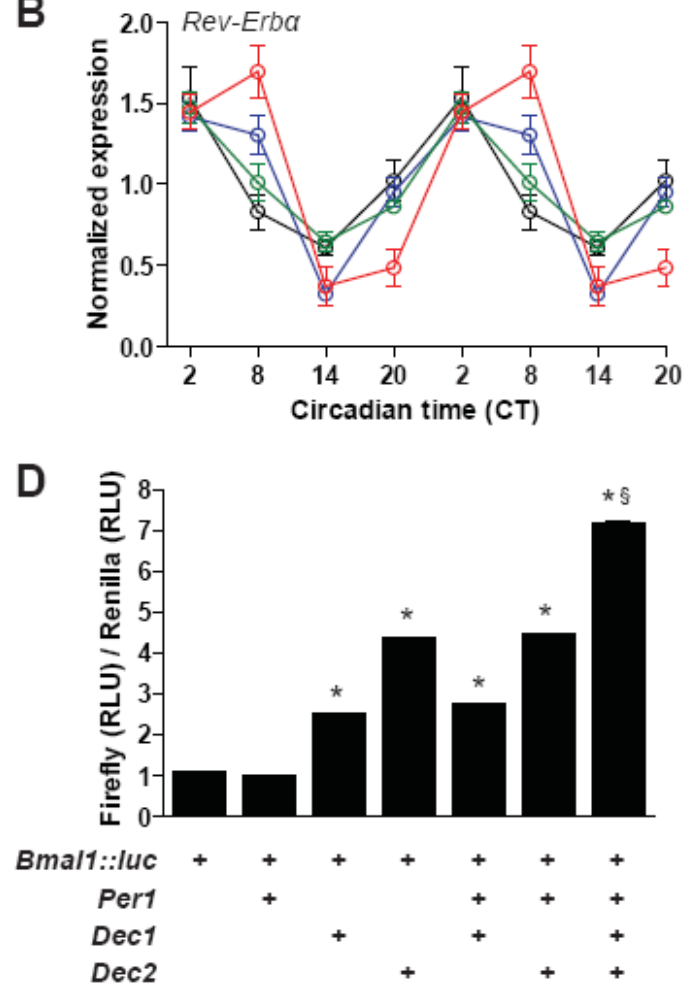


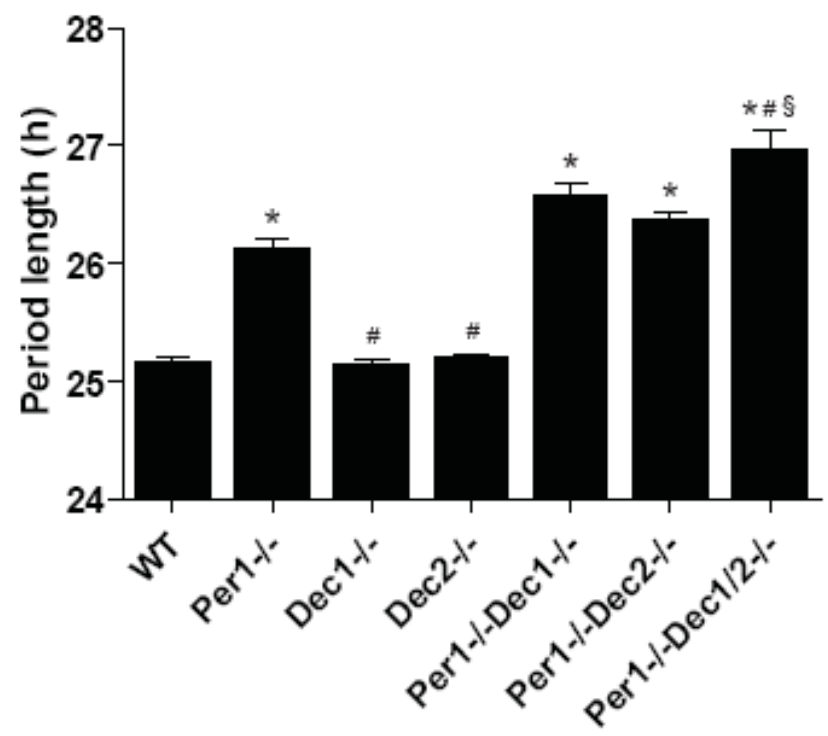

Bode et al. Figure S1 


$$
\begin{array}{ll}
\rightarrow \text { WT } & \rightarrow \text { Dec1\%- } \\
\text { ๑ Per1-/ } & \text { - Dec } 2 \%
\end{array}
$$

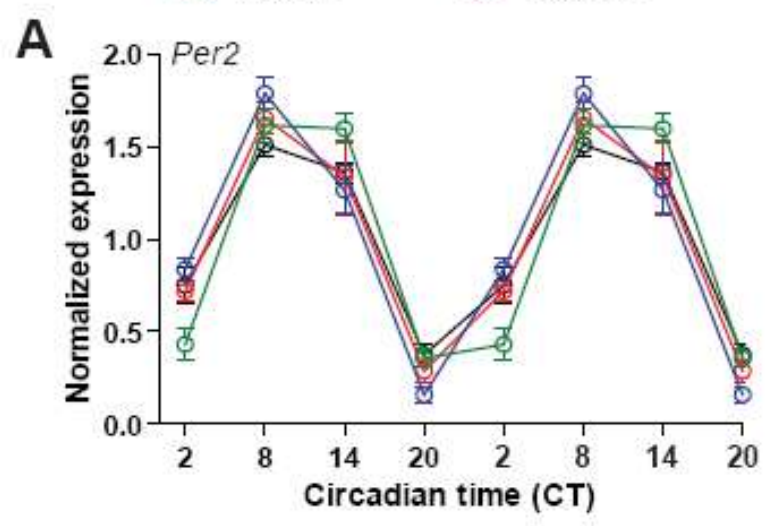

B $2.07^{\text {Bmal1 }}$

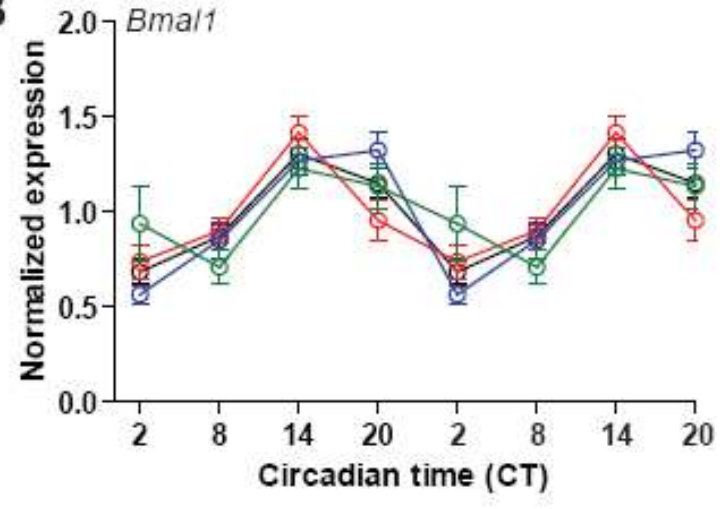

C 2.07 Rev-Erba

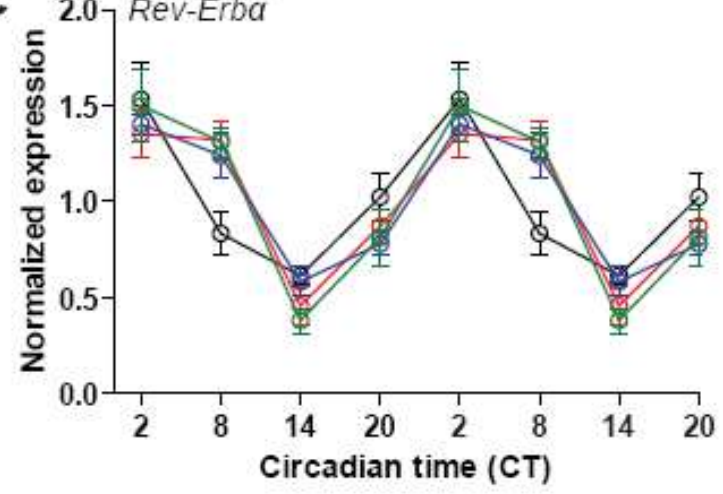


Table S1: Statistics of behavioral paradigms

\begin{tabular}{|c|c|c|c|c|c|c|c|c|}
\hline Tested parameter & $\begin{array}{l}\text { Genotype } \\
\text { comparison }\end{array}$ & $\downarrow \rightarrow$ & Per1-/- & Dec1- & Dec2- - & Per1-/-Dec1-/- & Per1-/-Dec2- - - & Per1-/-Dec1/2-/- \\
\hline Onset in LD & 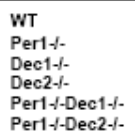 & & $m$ & $\begin{array}{l}\text { ns } \\
\text { ns }\end{array}$ & $\begin{array}{c}\mathrm{ns} \\
\mathrm{x} \\
\mathrm{x}\end{array}$ & $\begin{array}{l}m x \\
m x \\
m x\end{array}$ & $\begin{array}{l}m \\
n s \\
m \\
m\end{array}$ & $\begin{array}{l}m \\
m \\
m \\
m \\
m \\
m\end{array}$ \\
\hline Onset variation in LD & 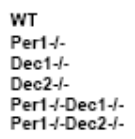 & & $x$ & $\begin{array}{l}\text { ns } \\
\text { ns }\end{array}$ & $\begin{array}{l}\mathrm{ns} \\
\mathrm{ns} \\
\mathrm{ns}\end{array}$ & $\begin{array}{l}m \\
m \\
m\end{array}$ & $\begin{array}{l}\mathrm{nn} \\
\mathrm{ns} \\
\mathrm{ns} \\
\mathrm{ns}\end{array}$ & $\begin{array}{l}m \\
m \\
m \\
m \\
m \\
m\end{array}$ \\
\hline Period length in DD & 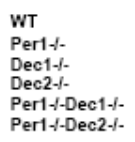 & & $\mathrm{xx}$ & $\begin{array}{l}m x \\
m x\end{array}$ & $\begin{array}{l}\mathrm{ns} \\
\mathrm{m} \\
\mathrm{m}\end{array}$ & $\begin{array}{l}\mathrm{mx} \\
\mathrm{ns} \\
\mathrm{mx}\end{array}$ & $\begin{array}{l}\mathrm{m} \\
\mathrm{ns} \\
\mathrm{m} \\
\mathrm{ns}\end{array}$ & $\begin{array}{l}m x \\
m \\
m \\
m \\
m \\
m\end{array}$ \\
\hline Period length variation in $\mathrm{DD}$ & 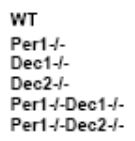 & & * & $\begin{array}{l}\text { ns } \\
\text { ns }\end{array}$ & $\begin{array}{l}\text { ns } \\
\text { ns } \\
\text { ns }\end{array}$ & $\begin{array}{l}\mathrm{mx} \\
\mathrm{ns} \\
\mathrm{x}\end{array}$ & $\begin{array}{l}m \\
n s \\
n \\
n s\end{array}$ & $\begin{array}{l}m \\
n s \\
m \\
m \\
n s \\
n s\end{array}$ \\
\hline Period length in LL & 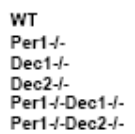 & & $x x$ & $\begin{array}{l}\mathrm{ns} \\
\mathrm{nx}\end{array}$ & $\begin{array}{l}\mathrm{ns} \\
\mathrm{ns} \\
\mathrm{ns}\end{array}$ & $\begin{array}{l}\mathrm{mx} \\
\mathrm{ns} \\
\mathrm{nx}\end{array}$ & $\begin{array}{l}\mathrm{m} \\
\mathrm{ns} \\
\mathrm{ns} \\
\mathrm{ns}\end{array}$ & $\begin{array}{l}m \\
m \\
m \\
m \\
m \\
n s\end{array}$ \\
\hline
\end{tabular}

ns $=$ not significant $\quad * p \leq 0.05 \quad * * p \leq 0.01 \quad * 0.001$ 
Table S2: Statistics of reporter gene assay

\begin{tabular}{|c|c|c|c|c|c|c|c|}
\hline Tested parameter & $\begin{array}{l}\text { Expression plasmid } \downarrow \rightarrow \\
\text { comparison }\end{array}$ & $\begin{array}{l}\text { Bmal1::luc, } \\
\text { Per1 }\end{array}$ & $\begin{array}{l}\text { Bmal1::luc, } \\
\text { Dec1 }\end{array}$ & $\begin{array}{l}\text { Bmal1::luc, } \\
\text { Dec2 }\end{array}$ & $\begin{array}{l}\text { Bmal1::luc, } \\
\text { Per1, Dec1 }\end{array}$ & $\begin{array}{l}\text { Bmal1::luc, } \\
\text { Per1, Dec2 }\end{array}$ & $\begin{array}{l}\text { Bmal1::luc, } \\
\text { Per1, Dec1, Dec2 }\end{array}$ \\
\hline Reporter gene assay & $\begin{array}{l}\text { Bmal1::luc } \\
\text { Bmal1::luc, Per1 } \\
\text { Bmal1::luc, Dec1 } \\
\text { Bmal1::luc, Dec2 } \\
\text { Bmal1:: luc, Per1, Dec1 } \\
\text { Baml1::Iuc, Per1, Dec2 }\end{array}$ & ns & $\begin{array}{l}m x \\
m x\end{array}$ & $\begin{array}{l}m \\
m x \\
m \\
m\end{array}$ & $\begin{array}{l}m \\
m \\
n s\end{array}$ & $\begin{array}{l}m \\
m \\
m \\
m \\
m\end{array}$ & $\begin{array}{l}m \\
m \\
m \\
m \\
m \\
m\end{array}$ \\
\hline
\end{tabular}

ns $=$ not significant $\quad * p \leq 0.05 \quad * * p \leq 0.01 \quad * * 0.001$ 
3.2. 'Advanced Activity Phase Entrainment and Restored FreeRunning SCN Rhythms in Per2/Dec Mutant Mice' (submitted to Chronobiology International)

Brid Bode, Moritz J. Rossner, Henrik Oster 


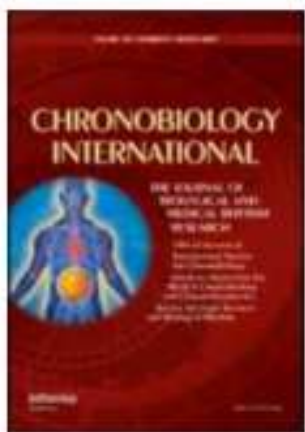

Advanced Activity Phase Entrainment and Restored FreeRunning SCN Rhythms in Per2/Dec Mutant Mice

\begin{tabular}{|c|c|}
\hline Journal: & Chronobiology International \\
\hline Manuscript ID: & Draft \\
\hline Manuscript Type: & Original Reports \\
\hline $\begin{array}{r}\text { Date Submitted by the } \\
\text { Author: }\end{array}$ & $n / a$ \\
\hline Complete List of Authors: & $\begin{array}{l}\text { Bode, Brid; Max Planck Institute for Biophysical Chemistry, } \\
\text { Crcadian Rhythms Group } \\
\text { Rossner, Moritz; Max Planck Institute for Experimental Medicine } \\
\text { Oster, Hennik; Max Planck Institute for Biophysical Chemistry, } \\
\text { Circadian Rhythms Group }\end{array}$ \\
\hline Keywords: & Per2, Dec1, Dec2, entrainment, masking, SCN, mice \\
\hline
\end{tabular}

\section{SCHOLARONE"}

Manuscripts 
Page 1 of 27

1

4

5

6
7

8

9
10

11

12

13
14

15

16
17

18

19

20

22

23

24

25

27

28

30

31

32

33

34
35

36

37

38

40

41

42

43

44

45

47

48

49

50

52
53

54

55

56
57

58

59

60

URL: http:llme manuscriptcentral.com/cbl E-mall: USA michat.h Germany 2012705)
Chronobiology International

Bode et al. - Per2-Dec Circadian Interoction

\begin{abstract}
Advanced Activity Phase Entrainment and Restored Free-Running SCN Rhythms in
\end{abstract} Per2/Dec Mutant Mice

Brid Bode', Moritz J. Rossner', Henrik Oster,"

${ }^{1}$ : Circadian Rhythms Group, Max Planck Institute for Biophysical Chemistry, 37077 Götingen,

2: Max Planck Institute for Experimental Medicine, 37075 Göttingen. Germany

*. corresponding author (henrik.oster mpibpe.mpg,de: tel ++49-551-2012738; fax ++49-551-

1 


\begin{abstract}
Many behavioral and physiological processes display diumal ( 24 hour) thythms controlled by an internal timekeeping system - the circadian clock. In mammals, a circadian pacemaker is located in the suprachiasmatic nuclei ( $\mathrm{SCN}$ ) of the hypothalamus and synchronizes peripheral escillators found in most other tissues with the external light-dark cycle. At the molecular level, circadian clocks are regulated by transcriptional translational feedback loops (TTLs) involving a set of clock genes. The mammalian core TTL includes the transcriptional modulators PER (1-3) and CRY (1/2) that inhibit their own expression by interaction with CLOCK/NPAS2 and BMAL1 (ARNTL). The basic helix-loop-helix transcription factors DECl (BHLHEA0) and DEC2 (BHLHE41) can interact with this con TTL, forming an accessory foedback mechanism. We measured circadian locomotor behavior and clock gene expression in the SCN of Per $2 / \mathrm{DeC}$ double and triple mutant mice to analyze the functional interaction of PER2 and DEC feedback on circadian pacemaker function in the SCN. Our wheel-running data suggest a synergistic function of Per2 and $\mathrm{Dec} / / 2$ in activity entrainment to a standard light/dark (LD) cycle, correlating with a cumulative deficiency in negative masking capacities in Per2/Dec double and triple mutant mice. In contrast, under constant darkness (DD) conditions, a deletion of either Decl or Dec2 partially rescued the Per2 mutant short period/arthythmicity phenotype. accompanied by a restoration of clock gene rhythms in the SCN. Together, our results show a strong interaction of Per2 and Decl/2 feedback proeesses in the SCN with differential modes of interactivity under entrained and free-run conditions.
\end{abstract}

Key words: Per2, Decl, Dec2, entrainment, masking, SCN, mice 


\section{INTRODUCTION}

Environmental conditions on Earth are characterized by $24 \mathrm{~h}$ cycles brought about by our planet's rotation around its own axis. In most species internal timekeepers, so called circadian clocks, have evolved enabling an organism to anticipate and efficiently adapt behavior and physiology to these predictable changes (Green et al. 2008; Takahashi et al. 2008). In mammals these clocks are based on interlocked transcriptional translational feedback loops (TTLs) comprised of a set of clock genes including Per(iod) 1-3, Cry(ptochrome) 1-2, Clock and Bmall (Arnt) (Ko and Takahashi 2006). The latter two transcription factors activate expression of Pers and Crys via E-box promoter elements in the SCN during the day. PER/CRY protein complexes relocate back into the nucleus where they interfere with CLOCK/BMAL1, suppressing their own transcription during the course of the night (Griffin et al. 1999: Kume et al. 1999; Reppent and Weaver 2002). Recently two other transeriptional regulators, DEC1 (BHLHE40) and DEC2 (BHLHE41), have been shown to interfere with the circadian clock machinery (Alvarez and Sehgal 2002). Both genes are rhythmically expressed in the mammalian circadian pacemaker, the suprachiasmatic nucleus (SCN) (Honma et al. 2002; Noshiro et al. 2005). Dec gene transcription is activated by CLOCK/BMALI via cis-regulatory E-box elements (Hamaguchi et al. 2004; Kawamoto et al. 2004). Further, Decl activity is highly light-responsive and has, thus, been implicated in light resetting of the SCN clock (Honma et al. 2002; Rossner et al, 2008), Both DEC proteins can modulate E-box-mediated transcription and directly interact with BMALI (Honma et al. 2002; Sato et al. 2004). Dec//2 single and double deficient mice show moderate alterations in clock period regulation and light resetting (Rossner et al. 2008). Interestingly, cell culture-based studies suggest that both DECs and PERs do not exclusively act as transcriptional repressors of E-boxcontrolled genes, depending on cell type and/or the presence of BMALI (Azmi et al, 2003; Dardente et al. 2009; Hamaguchi et al, 2004; Honma et al, 2002; Kawamoto et al. 2004; Li et al. 2004; Li et al. 2003; Rossner et al. 2008; Shearman et al. 2000; Theng et al. 2001; Zheng et al. 1999). To better understand a potential interactivity between Per2 and Dec in the regulation of the SCN pacemaker we have generated $\mathrm{Per} 2 / \mathrm{Dec} / 2$ double and triple mutant mice to analyze behavioral and molecular effects of Per $2 / \mathrm{Dec}$ deficiency on SCN clock function. Strong synergistic effects were observed between $\operatorname{Per} 2$ and $\operatorname{Dec} / / 2$ in activity phase entrainment, while free-running period length was determined by Per 2 function and partially rescued in the absence 
Bode et al, - Per2-Dec Circadian Interaction

of one Dec gene. Antagonistic interaction was observed in rhythm stabilization at behavioral and molecular levels under constant darkness conditions, suggesting that extemal environmental conditions (i.e. light) affect Per2-Dec cross-talk in the SCN.

URL: http:Iimc.manuscriptcentral.com/lcbi E-mail: USA: michael.h.smolensky@uth.tmc.edu/ EUROPE: touitou@ecr.jussieu.fr 


\title{
MATERIALS AND METHODS
}

\begin{abstract}
Animals

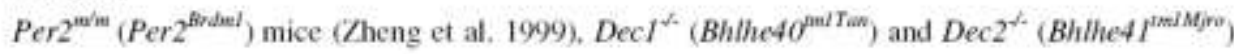
mice (Rossner et al. 2008; Sun et al. 2001) were backcrossed to a C57BL/6J background and then

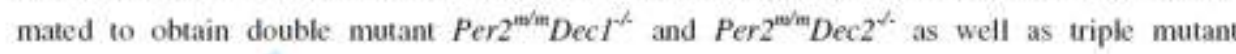
Per $2^{\text {m/m }}$ Decl/2 $2^{*}$ mice, The genotype of the offspring was determined by PCR as described (Rossner et al. 2008; Zheng et al. 1999), All experiments were performed on male adult animals (2-10 months), C57BI/6J mice were used as controls. All animal experiments were done with prior permission from the Office of Consumer Protection and Food Safety of the State of Lower Saxony and in accordance with the German Animal Welfare Act:
\end{abstract}

\section{Behavioral analysis}

Mouse housing and handling were performed as described (Albrecht and Oster 2001). Wheelrunning activity was analyzed using ClockLab software (Actimetrics, Evanston, IL). Prior to the experiments, animals were entrained to a 12 hours light: 12 hours dark (LD) cycle for at least 10 days (light intensity 250 lux). For analyzing free-running locomotor activity under constant darkness conditions (DD), lights wene turned off at the end of the light phase (Zeitgeber time. ZT12) and not turned on again on the next day. Activity profiles in LD and DD were assessed over a time of $4-7$ consecutive days. Onset phase angles were determined by fitting a straight line to 7-10 consecutive onsets using ClockLab software and manual correction. Onset variation depicts the mean deviation of neal activity onsets from a regression line through 7-10 consecutive onsets under stably entrained or free-running conditions, Rhythmicity and period length ( $\tau$ ) in DD were determined by $\chi^{2}$ periodogram analysis over an interyal of 7-10 consecutive days. To lest for masking capacities animals were entrained to a LD cycle for 10 days. A 60 min light pulse (100 lux) was applied between ZT14 and ZT15 on day 10. The relative light phase activity was calculated as the wheet-running activity during the light exposure period on day 10 divided by the average wheet-running activity without light treatment on days $7-9$ for the same animal at ZT14-15. To analyze acute phase shifting by single nocturnal light pulses animals were entrained to a LD cycle for al least two weeks. A 15 min light pulse (250 lux) was applied at ZT14 before animals were released into DD. Phase shifts were determined as the difference between the 
regression lines through onsets before and after the light pulse on the first day after the light exposure as compared to animals of the same genotype which were released into DD without extra light treatment.

\section{In situ hybridization}

Animals were entrainted to LD for 10 days, released into DD and sacrificed on the second day at circadian times CT2, CT8, CT14 and CT20. Brains were dissected under dim red light, fixed, dehydrated and paraflin embedded. $8 \mu \mathrm{m}$ sections were hybridized with ${ }^{35} \mathrm{~S}$-UTP-labeled antisense RNA probes for clock gene transcripts as described (Oster et al. 2003b; Oster et al. 2002). The Rev-Erba probe template was generated by PCR (forward primer: CCCTCTACAGTGACAGCTCCA, reverse primer: TCAGACACCGTTTGTACTGGA) from murine adipose tissue cDNA. Relative quantification of expression levels was performed by densitometric analysis of autoradiograph films using Quantity One software (Bio-Rad, Munich. Germany), SCN data were adjusted to the optical density measured in the lateral hypothalamus next to the SCN. For each experiment 3 animals per genotype were used and 3 adjacent SCN sections per animal were analyzed.

\section{Statistical analỵsis}

Statistical analyses were performed using GraphPad Prism software (GraphPad, La Jolla, CA). Groups were compared by One-way ANOVA followed by Tukey's post test for multiple comparisons. A p-value $<0.05$ was considered significant. The rhythmicity of clock gene expression was evaluated by CircWave v1.4 (Oster et al. 2006). 
All strains were backcrossed for more than ten generations to a C57BL/6J background prior to

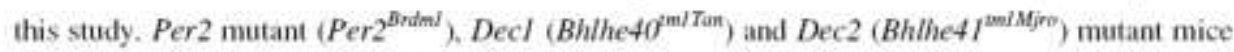
were mated to yield Per2/Dec double and triple mutant animals. All genotypes were bom at the expected Mendelian ratios, were viable and fertile and fid not show any gross morphological and behavioral abnomalities (data not shown). Adult males (2-10 months) were singly housed in running-wheel equipped cages and analyzed for locomotor behavior under defined light conditions. Under $12 \mathrm{~h}: 12 \mathrm{~h}$ light dark (LD) conditions all animals entrained readily to the LD cycle with locomotor activity being predominantly confined to the dark phase (Fig. 1A-G). As reported before Per 2 mutant mice displayed a marked phase advance of activity onsets preceding the beginning of the dark phase by $0.6 \mathrm{~h}$ (Fig. IH: Zheng et al. 1999). No significant phase advances were seen in Dec single mutants (Rossner et al, 2008), while activity onsets were dramatically advanced ( $1 \mathrm{~h}$ and more) in Per2/Dec double and triple mutant animals (Fig. IH and statistics in supplemental Table 1). These phase angle advances were correlated to an increasing imprecision in activity onsets in double and triple mutant mice (Fig. II). While wild-type animals showed very predictable activity onsets in LD with day-to-day variations of less than $0.1 \mathrm{~h}$, this variation was increased to $0.3 \mathrm{~h}$ in Per2 single mutants and further to more than $0.4 \mathrm{~h}$ in Per2/Dec double and triple mutant animals (Fig. II and statistics in supplemental Table 1).

When released into constant darkness conditions (DD) all genotypes showed circadian rhythms of activity (Fig. IA-G). Period length ( $t$ ) was $23,5 \mathrm{~h}$ in wild-type animals. As reported befone period length was significantly shortened to $21.9 \mathrm{~h}$ in Per2 mutant animals (Fig. 2A; Zheng et al. 1999). A moderate period reduction of $0.5 \mathrm{~h}$ was also seen in Decl deficient mice while Dec2 mutants did not show any significant period phenotype (Fig. 2A: Rossner et al. 2008). In Per $2 / D e c$ double and triple mutant animals period lengths fell between those seen in the single mutant mice, but were consickrably closer to Per2 mutants (Fig. 2A and statistics in supplemental Table 1). It had been reported that Per2 mutant mice show unstable circadian fhythms and become completely arthythmic upon extended time in DD (Zheng et al. 1999). To test if rhythm robustness was affected in Per $2 / D e c$ mutant animals we kept a subset of animals under DD conditions for more than three weeks, While the majority Per2 mutants had lost their circadian activity thythm by that time, Per2/Decl and Per $2 / \operatorname{Dec} 2$ double mutants showed a

URL: http://mc.manuscriptcentral.com/lcbi E-mail: USA: michael.h.smolensky@uth.tmc.edu/ EUROPE: touitou@ccr.jussieu.fr 
marked rescue in rhythm sustainability with more than $60 \%(\operatorname{Per} 2 / \operatorname{Dec} l)$ and $90 \%(\operatorname{Per} 2 / \operatorname{Dec} 2)$ of animals retaining thythmicity after 21 days in DD (Fig. 2B). This reseue effect seems to depend on the presence of at least one functional Dec gene as Per $2 / D e c l / 2$ triple mutants showed thythm sustainability similar to Per2 mutant animals (Fig. 2B).

To test if this behavioral interaction was reflected at the molecular level we analyzed the circadian expression of the clock genes Perl, Bmall and Rev-Erba (NrIdl) in the SCN of Per $2 /$ Dec mutant animals on the second day in DD using radioactive in situ hybridization on coronal brain sections. In wild-type animals Per I, Bmall and Rer-Erba mRNAs showed robust circadian thythms of expression with peak kevels at Circadian time (CT) 8, 14 and 2, respectively (Fig. 3A-C). As reponted before, the expression thythms of all three genes were severely blunted in Per2 mutant mice (Zheng et al. 2001: Theng et al. 1999). While only minor effects on expression rhythms were seen in Dec single mutant animals (Suppl. Fig. 1A-C). rescued Inanscription thythms wen observed for Perl and Bmall in Per2/Decl and Per2/Dec2 double mutants and for Rev-Erba in Per2Dec2 mutants (Fig. 3A-C), correlating with the partial rescue of thythm sustainment and period length in these animals (Fig, 2A, B). Of note, in comparison to wild-types, Perl and Bmall peak activities were phase advanced in Per2/Dec double mutants (Fig. 3A. B) while Rev-Erba expression was phase delayed in Per2/Dec2 mutant mice (Fig. 3C). Together, these data indicate that the observed alterations in behavioral rhythm robustness ane the direct consequence of changes in TTL transcription amplitudes in the SCN.

This finding prompted us to ask if the observed changes in activity onsets in Per $2 / D e c$ double and triple mutants (Fig $1 \mathrm{H}$ ) are related to alterations in the entrainment capacity of the SCN pacemaker to light. A short 15 min light pulse delivered at ZT14 on the last day in LD (Aschoff type II protocol) caused a $1.3 \mathrm{~h}$ delay in activity onsets in wild-type animals when compared to non light-exposed controls (Fig. 4A and statistics in supplemental Table 1). As previously reported this photic phase resetting was blunted in Per2 mutant animals $(0.5 \mathrm{~h})$ (Albrecht et al. 2001). Decl single. Per $2 / \mathrm{Decl}$ double and Per2/Decl/2 triple mutants showed a moderate reduction in phase delays $(0.9-1$ h) while a normal phase resetting capacity was observed in Dec2 mutant animals ( $1.2 \mathrm{~h})$ (Fig. $4 \mathrm{~A}$ and statistics in supplemental Table 1). Of note, Per $2 / \operatorname{Dec} 2$ double mutant mice displayed even stronger photic phase delays than wild-type controls (1.8 vs. 1.3 h). In summary, the effects of $\operatorname{Per} 2 / \mathrm{Dec}$ deficiency on light-mediated resetting of the circadian clock were less uniform and did not match the changes observed in LD onset behavior. 
Therefore, we asked if the advanced activity onsets might reflect a decrease in acute behavioral responses to light exposure (so called masking) (Mrosovsky 1999). To test this we subjected stably entrained animals to a $1 \mathrm{~h}$ light pulse (100 lux) during the first half of the dark phase (ZT 14-15) and compared activity levels to those observed on previous days at the same time interval. While wild-type and single mutant mice showed a robust suppression of activity levels by $70-80 \%$ under 100 lux illumination, masking was significantly impaired in Per $2 / \mathrm{Dec}$ double mutants. The same trend was seen in Per2Decl/2 triple mutants (Fig. $4 \mathrm{~B}$ and statisties in supplemental Table 1). These data closely correlate with the onset phenotypes observed in these animals (Fig. IE-H). Hence, the activity onset regulation under LD conditions appears to primarily reflect changes in negative masking capacities in Per2/Dec mutant mice rather than changes in regulations of photic TTL resetting. 
Bode et al. - Per2-Dec Circadian Imeraction

\section{DISCUSSION}

In this study we analyzed Per2-Dec interaction in the regulation of molecular and behavioral circadian rhythms in mice. We found that $P e r 2$ and $D e c I / 2$ synergistically affect the regulation of activity entrainment, possibly via the regulation of negative masking. Under free-running conditions, however, deletion of $\mathrm{Decl}$ or $\mathrm{Dec} 2$ can partially rescue the Per2 mutant phenotype for period length and rhythm stability. This effect is associated with changes in clock gene activity rhythms in the SCN pacemaker.

Activity phase angles under entriáned conditions are often proportional to the difference between external and internal period length, thus depending on the daily phase shifting limits of the external light/dark cycle (Brown et al. 2008). Shortened periodicity in combination with reduced capacity for phase delays was also suggested to underlie the early activity onsets observed in Per2 mutant mice (Spoelstra et al. 2004). While in this study in the single mutant animals masking was preserved (Fig. 4B) and, thus, seems not to affect predark activity, period length regulation and phase onset effects were clearly dissociated in Per $2 / \mathrm{Dec}$ double and triple mutant mice (Fig. 1H, L, 2A), In these animals, negative masking, but not phase shifting capacity, correlates closely with onset phase angle alterations (Fig. 4). This suggests that the early onset observed under LD conditions might to a large part result from ineffective acute photic suppression of locomotor activity during the late day. Both Per2 and the Decs seem to be involved in this regulation. Interestingly, a close relationship between period length and activity phase in humans has so far only been reported for familiar advanced sleep phase syndrome (FASPS) patients that carry mutations diretly or indirectly affecting PER2 stability and turnover (Jones et al. 1999: Toh et al. 2001). Other chronotypes affecting gene polymorphisms have been described that do not show significant alterations in frec-running period (Archer et al. 2003; Ebisawa et al. 2001: Viola et al. 2007) and, thus, appear to rather reflect changes in photic input to the clock of to other arousal-regulating centers in the brain. An elegant cell-based study by Brown and colleagues shows that entrainment phase changes in humans can also result from changes in the molecular robustness of the cellular TTL machinery (Brown et al. 2008), In line with this, our in situ data suggest that in Per 2 single and Per2/Dec double mutants clock gene oscillations in the SCN are disrupted (Fig. 3). Interestingly, this results in an increased sensitivity of the SCN clock to acute nocturnal light pulses in Per2Dec2 double mutant mice, but not in

10

URL: http://mc.manuscriptcentral.com/lcbi E-mail: USA: michael.h.smolensky@uth.tmc.edu/ EUROPE: toultou@ecr.jussieu.fr 

resetting of entrainment phase. Per2 and Dec2 might be involved in the regulation of resetting.

Per2Decl mutants (Fig. 4A). Given the changes in base TTL function brought about by the functional deletion of single components it is possible that the integrated light response characteristics over the course of the day are altered in these mutants, resulting in a modified

Photic phase nesetting of the circadian clock is believed to depend on Perl and Per2 (and possibly Dec1) induction in the SCN after light exposure during the subjective night (Albrecht et al. 1997: Honma et al. 2002: Miyake et al. 2000: Rossner et al. 2008: Shearman et al. 1997: Shigeyoshi et al, 1997; Yan and Silver 2002). In line with this Perl and Per2 single mutant mice show decreased behavioral responses to nocturnal light exposure (Fig, 4A; Albrecht et al. 200I: Spoelstra et al. 2004). Phase resetting in Decl single and Per2/Decl double mutants is also affected, indicating that besides Per2, Decl is more important for light resetting than Dec2, in line with the fact that only Decl is acutely light-inducible in the SCN (Honma et al, 2002). However, the increased phase shifts of Per $2 / D e c 2$ mutant mice suggest that an interaction of

Under free-running conditions period length and rhythm stability are strongly affected by a Per 2 mutation, resulting in gradual arrtythmicity under DD conditions (7heng et al. 1999). This mythm deterioration effect is partly rescued by an additional deletion of Decl or Dec2 in these mice, along with a partial restoration of period length and clock gene expression rhythms in the SCN (Fig. 2 and Fig. 3). In the absence of both Dees and Per2, however, the two effects are neutralized, indicating a certain functional redundancy of both Decs together with an antagonistic Per2-Dec interaction in free-run. Similar, though more dramatic, effects have been observed in Per2-Cry interaction. While Per2/Cry2 double mutant animals show full period length and thythmicity reseue (Oster et al. 2002), Per2/Cryl/2 triple mutants ane fully arrhythmic (Oster et al. 2003a). It was suggested that $C r y / / 2$ redundancy might be involved in this process in a way that PER1-CRY1 complexes could compensate for loss of PER2 and CRY2 in the corresponding mutants (Oster et al. 2002). In the same way, Perl-Decl or Perl-Dec2 interaction could compensate for the absence of Per2 and one of the Decs in the double mutants. Whether this effect is based on physical interaction of the corresponding proteins or rather reflects a functional genetic interaction, e.g, via co-regulatory effects on target gene activation, remains to be shown. Our expression data clearly show that the deletion of $\mathrm{Decl}$ or $\mathrm{Dec} 2$ function in a Per2-deficient background results in a restoration of elock gene expression rhythms together with a general up- 
Bode et al. - Per2-Dec Circadian Interaction

regulation of transcriptional activity (Fig. 3). This indicates that, unlike Per2, the Dec genes are primarily acting as transcriptional activators in the SCN. This finding is supported by previous data from Dec//2 double deficient animals (Rossner et al. 2008). While little consequences on clock gene activity are seen in Dec single mutants, effects are more pronounced in the double and triple mutaut animals, indicating that the DEC proteins show a high grade of functional redundancy and - at least in the $\mathrm{SCN}$ - act primarily via interaction with PER(2). Transfection studies have suggested that the DECs might also have a function in transcriptional regulation of E-box genes independent of PER and CRY proteins (Azmi et al. 2003; Dardente et al. 2009: Hamaguchi et al. 2004: Honma et al. 2002; Kawamoto et al. 2004: Rossner et al, 2008). Our single mutant data, while not excluding this possibility for other tissues, clearly do not support such functionality for the SCN clock.

In the last years the chronobiological field has moved away from secing the clock machinery as a single TTL comprised of a limited number of transcriptional regulators. More and more acessory loops - some of them going beyond the simple concept of transcriptional translational feedbacks - have been described (Zhang and Kay 2010). It will be an important task for the future to unravel the interactivity of this network of feedback loops to start to understand the mechanistics behind the generation and stabilization of molecular circadian rhythms. The characterization of Per2-Dec interaction as presented in this study is a first step into this direction. providing new insight into the genetic basis of entrainment and the regulation of freerun activity patterns in the living animal. 
Page 13 of 27

1

3

4

5

6

10

11

14

15

16

18

19

20
21

22

23

24

26

27

28

29
30

31

32

33

34

35
36

37

38

39

41
42

43

44

45

47

48

49

50

51

53

54

55

56

57

58

59

60
Chronobiology International

Bode et al. - Per2-Dec Circadian Interaction

CONFLICT OF INTEREST

The authors report no conflicts of interest.

URL: http:/mc.manuscriptcentral.com/lcbi E-mail: USA: michael.h.smolensky@uth.tmc.edu/ EUROPE: touitou@ccr.jussieu.fr 
Bode et al. - Per2-Dec Circadian Interaction

\section{ACKNOWLEDGEMENTS}

We would like to thank Reshma Taneja for the generous gift of Decl mutant mice and Jana Husse and Insa Geffers for critical reading of the manuscript. This project was funded by a project grant (OS 353/2-1) and an Emmy Noether fellow ship (to H.O.) of the German Research Foundation (DFG).

URL: http://mc.manuscriptcentral.com/lcbi E-mait: USA: michael.h.smolensky@uth.tmc.edu/ EUROPE: touitou@ecr.jussieu.fr 
Bode et al - Per2-Dec Circadian Interaction

\section{REFERENCES}

Albrecht $\mathrm{U}$ and Oster $\mathrm{H}$ (2001) The circadian clock and behavior. Behavioural brain research 125:89-91.

Albrecht U. Sun ZS, Eichele G and Lee CC (1997) A differential response of two putative mammalian circadian regulators, mper $]$ and mper2, to light. Cell 91:1055-1064.

Albrecht U, Zheng B, Larkin D, Sun /S and Lee CC (2001) mPerl and mPer2 are essential for normal resetting of the circadian clock. J Biol Rhythms 16:100-104.

Alvarez D and Schgal A (2002) Circadian thythms: finer clock control. Nature 419:798-799.

Archer SN. Robilliard DL, Skene DJ. Smits M. Williams A. Arendt J and von Schantz M (2003) A length polymorphism in the circadian clock gene Per3 is linked to delayed sleep phase syndrome and extreme diumal preference, Sleep 26:413-415.

Azmi S, Sun H, Ozog A and Taneja R (2003) mSharp-1/DEC2, a basic helix-loop-helix protein functions as a transcriptional repressor of $\mathrm{E}$ box activity and Stral3 expression. The Journal of biological chemistry 278:20098-20109.

Brown SA, Kunz D, Dumas A, Westermark PO, Vanselow K. Tilmann-Wahnschaffe A, Herzel $\mathrm{H}$ and Kramer A (2008) Molecular insights into human daily behavior. Proceedings of the National Academy of Sciences of the United States of America 105:1602-1607,

Dardente H, Fustin JM and Hazlerigg DG (2009) Transcriptional feedback loops in the ovine circadian clock. Comparative biochemistry and physiology 153:391-398.

Ebisawa T, Uchiyama M. Kajimura N, Mishima K, Kamei Y, Katoh M, Watanabe T, Sckimote M, Shibui K, Kim K, Kudo Y, Ozeki Y, Sugishita M, Toyoshima R, Inoue Y, Yamada N, Nagase T. Ozaki N. Ohara O, Ishida N. Okawa M. Takahashi K and Yamauchi T (2001) Association of structural polymorphisms in the human period 3 gene with delayed sleep phase syndrome. EMBO reports $2: 342-346$

Green CB. Takahashi JS and Bass J (20008) The meter of metabolism. Cell 1,34:728-742.

Griffin EA, Jr., Staknis D and Weitz CJ (1999) Light-independent role of CRY1 and CRY2 in the mammalian circadian clock. Science 286:768-771.

Hamaguchi H. Fujimoto K. Kawamoto T. Noshiro M. Maemura K. Takeda N. Nagai R. Furukawa M, Honma S, Honma K. Kurihara H and Kato Y (2004) Expression of the gene for Dec2, a basic helix-loop-helix transcription factor, is regulated by a molecular clock system. Biochem J 382:43-50.

Honma S, Kawamoto T, Takagi Y. Fujimoto K, Sato F, Noshiro M. Kato Y and Honma K (2002) Decl and Dec2 are regulators of the mammalian molecular clock. Nature 419:841-844. 
Bode et al - Per2-Dec Circadian Interaction

Jakubcakova V, Oster H. Tamanini F, Cadenas C. Leitges M, van der Horst GT and Fichele G (2007) Light entrainment of the mammalian circadian clock by a PRKCA-dependent positranslational mechanism. Neuron 54:831-843.

Jones CR. Campbell SS, Zone SE, Cooper F, DeSano A, Murphy PJ, Jones B, Czajkowski L and Ptacek LJ (1999) Familial advanced slecp-phase syndrome: A short-period circadian rhythm variant in humans, Nature medicine 5:1062-1065.

Kawamoto T. Noshiro M. Sato F, Maemura K. Takeda N. Nagai R. Iwata T. Fujimoto K. Furukawa M. Miyazaki K. Honma S. Honma K and Kato Y (2004) A novel autofeedback loop of Decl transcription involved in circadian rhythm regulation. Biochemical and biophysical research communications $313: 117-124$

Ko $\mathrm{CH}$ and Takahashi IS (2006) Molecular components of the mammalian circadian clock. Human molecular genetics 15 Spec No 2:R271-277.

Kume K, Zylka MJ, Sriram S, Shearman LP, Weaver DR, Jin X, Maywood ES, Hastings MH and Reppert SM (1999) mCRY1 and mCRY2 are essential components of the negative limb of the circadian clock feedback loop. Cell 98:193-205.

Li Y, Song X, Ma Y, Liu J, Yang D and Yan B (2004) DNA binding, but not interaction with Bmall, is responsible for DECl-mediated transcription regulation of the circadian gene mPerl. Biochem . I382:895-904

Li Y, Xie M. Song X. Gragen S, Sachdeva K. Wan Y and Yan B (2003) DECl negatively regulates the expression of DEC2 through binding to the E-box in the proximal promoter. The Journal of biological chemistry 278:16899-16907.

Miyake S, Sumi Y. Yan L, Takekida S, Fukuyama T, Ishida Y. Yamaguchi S, Yagita K and Okamura H (2000) Phase-dependent responses of Perl and Per2 genes to a light-stimulus in the suprachiasmatic nucleus of the rat. Neuroscience letters 294:41-44.

Mrosovsky N (1999) Masking: history, definitions, and measurement. Chronobiology international 16:415-429.

Noshiro M. Furukawa M. Honma S, Kawamoto T. Hamada T. Honma K and Kato Y (2005) Tissue-specific disruption of rhythmic expression of Decl and Dec2 in clock mutant mice. $J$ Biol Rhythems 20:404-418

Oster H, Damerow S, Hut RA and Eichele G (2006) Transcriptional profiling in the adrenal gland reveals circadian regulation of hormone biosynthesis genes and nucleosome assembly genes. $J$ Biol Rhtwhms 21:350-361.

Oster H, van der Horst GT and Albrecht U (2003i) Daily variation of clock output gene activation in behaviorally arrhythmic mPer/mCry triple mutant mice. Chronobiology international 20:683-695.

16

URL: http://mc.manuscriptcentral.com/lcbi E-mail: USA: michael.h.smolensky@ith.tmc.edu/ EUROPE: touitou@ccr.jussieu.fr 
Oster H. Wemer C, Magnone MC. Mayser H. Feil R. Seeliger MW. Hofmann F and Albrecht U (2003b) cGMP-dependent protein kinase II modulates $m P e r l$ and $m P e r 2$ gene induction and influences phase shifts of the circadian clock. Curr Biol 13:725-733.

Oster H, Yasui A, van der Horst GT and Albrecht U (2002) Disruption of mCry2 restores circadian rhythmicity in $m$ Per 2 mutant mice, Genes Dev 16:2633-2638.

Reppert SM and Weaver DR (2002) Coordination of circadian timing in mammals. Nature 418:935-941.

Rossner M., Oster H, Wichert SP. Reinecke L, Wehr MC, Reinecke J, Eichele G, Taneja R and Nave KA (2008) Disturbed clockwork resetting in Sharp-l and Sharp-2 single and double mutant mice, PLOS ONE 3: 2762.

Sato F. Kawamoto T. Fujimoto K. Noshiro M. Honda KK. Honma S, Honma K and Kato Y (2004) Functional analysis of the basic helix-loop-helix transcription factor DECl in circadian regulation. Interaction with BMALI. European journal of biochemistry/FEBS 271:4409-4419.

Shearman LP, Sriram S, Weaver DR, Maywood ES, Chaves I, Zheng B, Kume K. Lee CC, van der Horst GT. Hastings MH and Reppert SM (2000) Interacting molecular loops in the mammalian circadian clock. Science 288:1013-1019.

Shearman LP. Zylka MJ, Weaver DR. Kolakowski LF, Jr. and Reppert SM (1997) Two period homologs: circadian expression and photic regulation in the suprachiasmatic nuclei. Neiron 19:1261-1269.

Shigeyoshi Y. Taguchi K, Yamamoto S, Takekida S, Yan L. Tei H. Moriya T, Shibata S. Loros JJ, Dunlap JC and Okamura $\mathrm{H}$ (1997) Light-induced resetting of a mammalian circadian clock is associated with rapid induction of the mPerl transcript. Cell 91:1043-1053,

Spoelstra K, Albrecht U, van der Horst GT. Brater V and Daan S (2004) Phase responses to light pulses in mice lacking functional per or cry genes, $J$ Biol Rhythms 19:518-529.

Sun H, Lu B, Li RQ, Flavell RA and Taneja R (2001) Defective T cell activation and autoimmune disorder in Stral3-deficient mice. Nature immunology 2:1040-1047.

Takahashi JS, Hong HK, Ko CH and McDearmon FL (2008) The genetics of mammalian circadian order and disorder: implications for physiology and disease. Nature reviews 9:764-775.

Toh KL, Jones CR, He Y, Eide EJ, Hinz, WA, Virshup DM. Ptacek LJ and Fu YH (2001) An hPer2 phosphorylation site mutation in familial advanced sleep phase syndrome. Science 291:1040-1043.

Viola AU, Archer SN, James LM, Groeger JA, Lo JC, Skene DJ, von Schantz M and Dijk DJ (2007) PER3 polymorphism predicts sleep structure and waking performance. Curr Biol 17:613618. 
Bode et al. - Per2-Dec Circadian Interaction

Yan L and Silver R (2002) Differential induction and localization of $\mathrm{mPerl}$ and $\mathrm{mPer} 2$ during advancing and delaying phase shifts. The European journal of neuroscience 16:1531-1540.

Zhang EE and Kay SA (2010) Clocks not winding down: unravelling circadian networks, Nat Rev Mol Cell Biol 11:764-776.

Zheng B, Albrecht U, Kaasik K. Sage M, Lu W, Vaishnav S, Li Q, Sun ZS, Eichele G, Bradley A and Lee CC (2001) Nonredundant roles of the mPerl and mPer 2 genes in the mammalian circadian clock. Cell 105:683-694

Zheng B, Larkin DW, Albrecht U, Sun ZS, Sage M, Eichele G, Lee CC and Bradley A (1999) The mPer2 gene encodes a functional component of the mammalian circadian clock. Nature 400:169-173. 


\section{FIGURE LEGENDS}

Figure 1. Locomotor behavior under entrained (LD) conditions. (A-G) Representative double plotted locomotor activity records of (A) wild-type (WT), (B) Per2 $2^{m / m}$. (C) $\mathrm{Decl} \mathrm{l}^{\mathrm{t}}$, (D) $\mathrm{Dec} 2^{4}$.

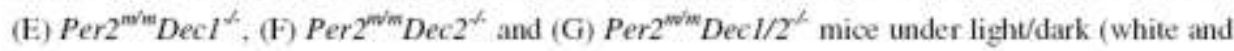
grey shaded, respectively) and constant darkness (grey shaded) conditions, (H) Quantified phase angle under light/dark (LD) conditions; wild-type (WT) $-0.04 \pm 0.01 \mathrm{~h}$ (n $=20$ ). Per $2^{\mathrm{m} / \mathrm{m}}$

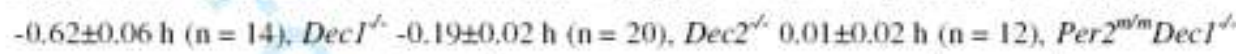
$-1.15 \pm 0.07 \mathrm{~h}(\mathrm{n}=12), \operatorname{Per} 2^{\mathrm{nin}} \operatorname{Dec} 2^{-}-1.26 \pm 0.08 \mathrm{~h}(\mathrm{n}=11)$ and $\operatorname{Per} 2^{\mathrm{m} / \mathrm{m}} \operatorname{Dec} 1 / 2^{/}-1.41 \pm 0.08 \mathrm{~h}$ $(n=6)$. (1) Quantification of onset variation under LD conditions: WT $0,05 \pm 0,004 \mathrm{~h}(\mathrm{n}=20)$,

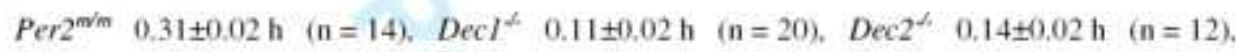

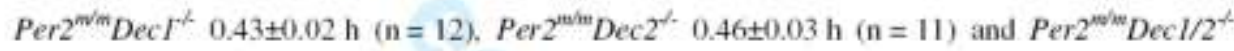
$0,55 \pm 0,06 \mathrm{~h}(\mathrm{n}=6)$. Data are represented as mean \pm SEM; $: \mathrm{p}<0,05$ compared to WT.

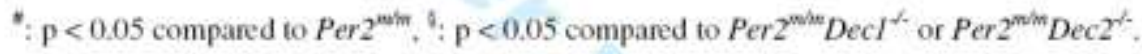

Figure 2. Locomotor behavior under free-running (DD) conditions. (A) Average frec-running period length in constant darkness (DD): WT $23.48 \pm 0.02 \mathrm{~h}(\mathrm{n}=20)$, Per2 $2^{\mathrm{m} / \mathrm{m}} 21.96 \pm 0.06 \mathrm{~h}$

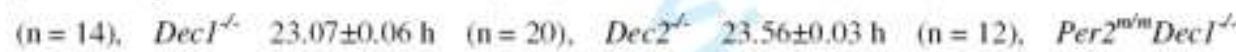

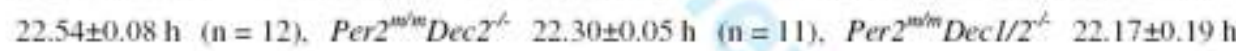
$(\mathrm{n}=6)$. Data are represented as mean \pm SEM: "; $p<0.05$ compared to $W T$, " $p<0.05$ compared

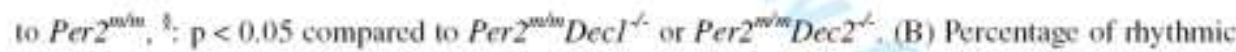
animals during 21 days in DD of wild-type (WT) Decl ${ }^{*}$, Dec2 $2^{*}$ (grey line; per genotype

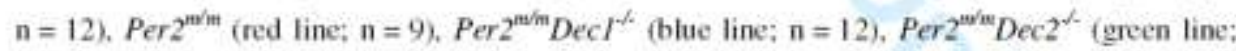

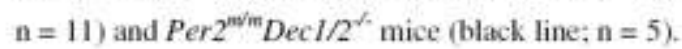

Figure 3. In situ hybridization (ISH) profiles of clock genes in the SCN. Normalized mRNA expression levels of (A) Perl, (B) Bmall and (C) Re1-Erba in the SCN under constant darkness

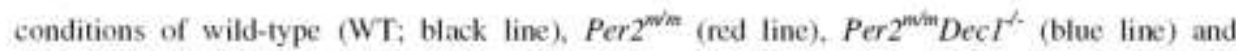
Per2 ${ }^{m i m} \operatorname{Dec}{ }^{*}$ (green line) mice $(\mathrm{n}=3$ ). Values are double plotted and represented as mean \pm SEM 
Bode et al - Per2. Dec Circadian Imeraction

Figure 4. Phase delay and masking responses to nocturnal light exposure. (A) Quantification of phase-delay responses after a $15 \mathrm{~min}$ light pulse at ZT14: WT $-1,26 \pm 0.08 \mathrm{~h}(\mathrm{n}=8)$, Per2 $2^{\mathrm{m} / \mathrm{t}}$

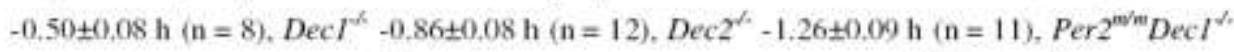

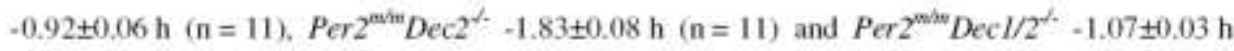
$(\mathrm{n}=5)$. (B) Relative light phase activity during light exposure (100 lux) at ZT14-ZT15: WT

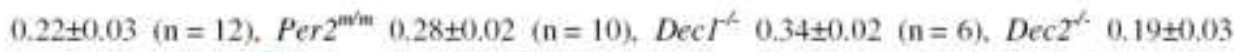

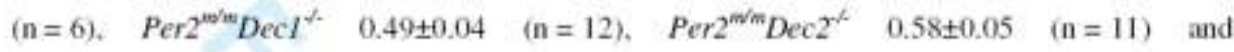
Per2 ${ }^{m / m} \mathrm{Decl} / 2^{*} 0.39 \pm 0.05(\mathrm{n}=5)$. Data are represented as mean \pm SEM; $;: p<0.05$ compared

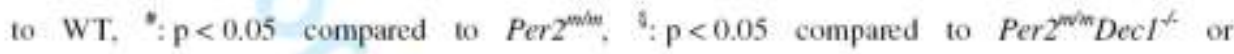
$\operatorname{Per} 2^{m i m} \operatorname{Dec} 2^{2}$.

Supplemental figure 1. In situ hybridization (ISH) profiles of clock gene expression in the SCN. Normalized expression of (A) Perl. (B) Bmall and (C) Rev-Erba mRNA in the SCN under constant darkness of wild-type (WT; black line), $\mathrm{Decl}^{+}$(blue line) and $\mathrm{Dec}^{\gamma /}$ (green line) mice $(n=3)$. Values are double plotted and represented as mean \pm SEM.

Supplemental figure 2. Phase shift responses to a nocturnal light pulse. (A-G) Representative double plotted actograms of activity responses following a 15 min light pulse at ZT14 (arrow) of

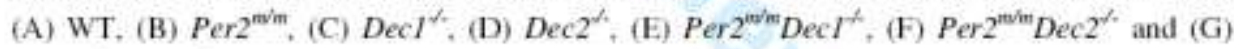
Per2 ${ }^{\text {mim }}$ Decl/2\% mutant mice. 

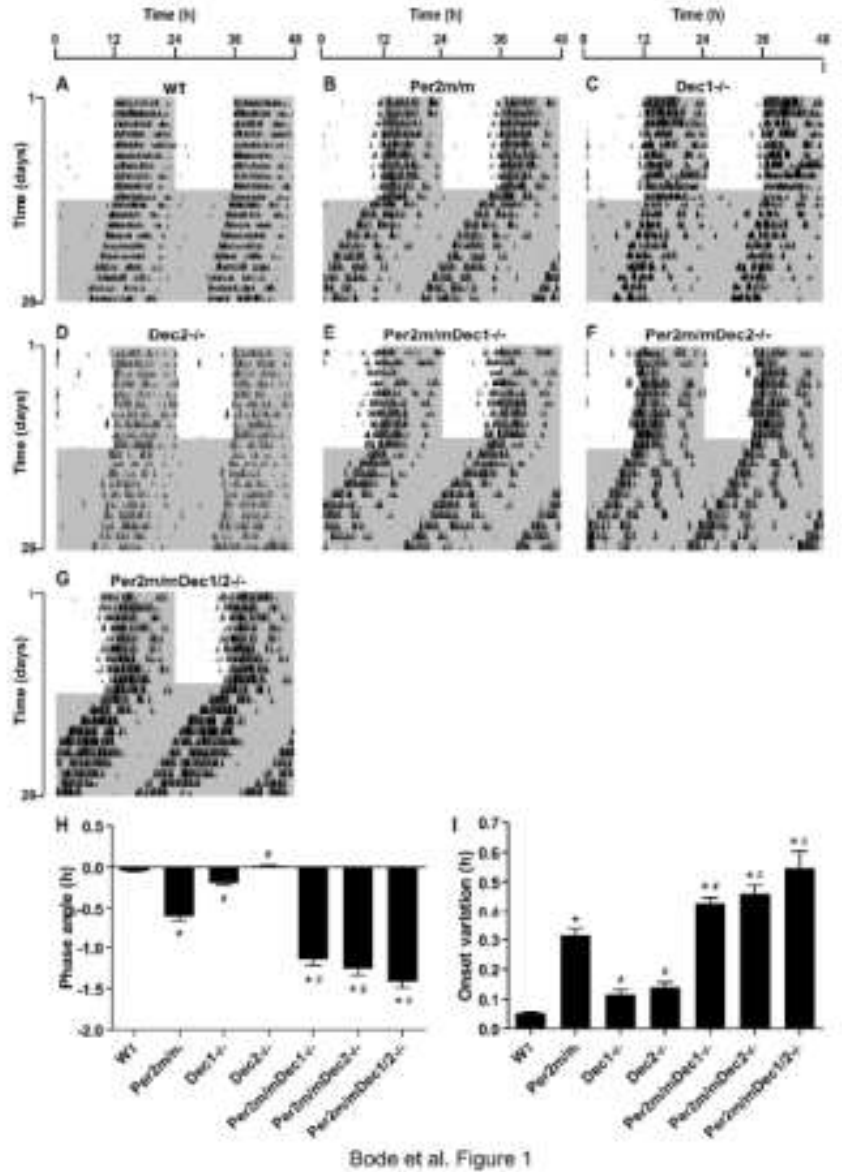

Locomotor behavior under entrained (LD) conditions, (A-G) Representative double plotted locomotor activity records of (A) wild-type (WT), (B) Per2m/m, (C) Dec1 (, (D) Dec2 /, (E) Per zm/mDec1 / (F) Per2m/mDec2 / and (G) Per2m/mDec1/2 / mice under light/dark (white and arey shaded, respectively) and constant darkness (arey shaded) conditions. $(H)$ Quantified phase angle under liaht/dark (LD) conditions: wild-type (WT) $-0.04 \pm 0.01 \mathrm{~h}(\mathrm{n}=20)$. Per $2 \mathrm{~m} / \mathrm{m} \quad 0.62 \pm 0.06 \mathrm{~h}(\mathrm{n}=14)$. Dec1/ $0.19 \pm 0.02 \mathrm{~h}\langle\mathrm{n}=20)$, Dec2 $30.01 \pm 0.02 \mathrm{~h}(\mathrm{n}=12)$, Per2m/mDec1/ $1.15 \pm 0.07 \mathrm{~h}(\mathrm{n}=$

12), Perzm/mDec2 / $1.26 \pm 0.08 \mathrm{~h}(\mathrm{n}=11)$ and Per $2 \mathrm{~m} / \mathrm{mDec} 1 / 2 / 1.41 \pm 0.08 \mathrm{~h}(\mathrm{n}=6)$. (I)

Ouantification of onset variation under 10 conditions: WT $0.05 \pm 0.004 \mathrm{~h}(\mathrm{n}=20)$, Per $2 \mathrm{~m} / \mathrm{m}$ $0.31 \pm 0.02 \mathrm{~h}(\mathrm{n}=14)$, Dec1 $/ 0.11 \pm 0.02 \mathrm{~h}(\mathrm{n}=20)$, Dec2 $/ 0.14 \pm 0.02 \mathrm{~h}(\mathrm{n}=12)$, Per $2 \mathrm{~m} / \mathrm{mDec} 1$ $/ 0.43 \pm 0.02 \mathrm{~h}(\mathrm{n}=12)$. Per $2 \mathrm{~m} / \mathrm{mDec} 2 / 0.46 \pm 0.03 \mathrm{~h}(\mathrm{n}=11)$ and Perzm/mDec $1 / 2 / 0.55 \pm 0.06$ $h(n=6)$. Data are represented as mean \pm SEM; *: $p<0.05$ compared to WT, $*: p<0.05$ compared to Per2m/m, 5 : $p<0.05$ compared to Per $2 \mathrm{~m} / \mathrm{mDec} 1$ / or Per2m/mDec2 $151 \times 216 \mathrm{~mm}(600 \times 600 \mathrm{DPI})$

URL: http:IImc.manuscriptcentral.com/lcbi E-mail: USA: michael.h.smolensky@uth.tmc.edu/ EUROPE: touitou@iccr.jussieu.fr 
URL: http://mc.manuscriptcentral.com/licbi E-mail: USA: michael.h.smolensky,guth.tmc.edu/ EUROPE: touitou@eccr.jussieu.fr

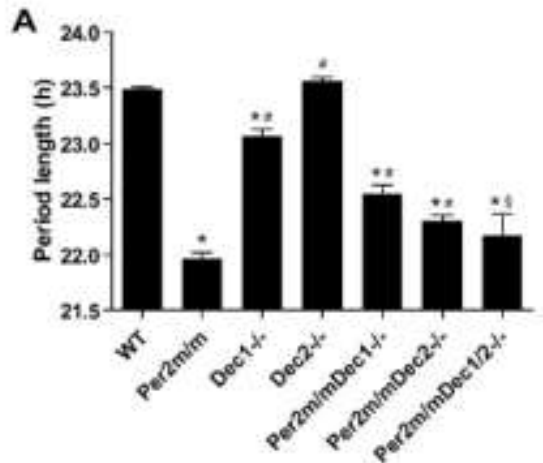

B

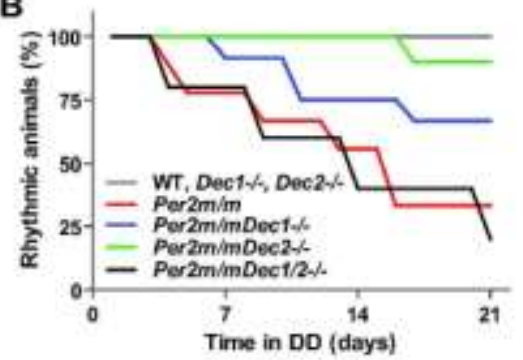

Bode et al. Figure 2

Locomotor behavior under free-running $(D D)$ conditions, (A) Average free-running period lenoth in constant darkness (DD): WT $23.48 \pm 0.02 \mathrm{~h}(n=20)$, Per $2 m / \mathrm{m} 21.96 \pm 0,06 \mathrm{~h}(n=14)$. Dec1 $23.07 \pm 0.06 \mathrm{~h}(\mathrm{n}=20)$, Dec2 $/ 23.56 \pm 0.03 \mathrm{~h}(\mathrm{n}=12)$. Per $2 \mathrm{~m} / \mathrm{mDec} 1 / 22.54 \pm 0.08 \mathrm{~h}(\mathrm{n}=12)$. Perzm/mDec2 / 22.30 $\pm 0.05 \mathrm{~h}(\mathrm{n}=11)$. Per $2 \mathrm{~m} / \mathrm{mDec} 1 / 2 / 22.17 \pm 0.19 \mathrm{~h}(\mathrm{n}=6)$. Data are represented as mean \pm SEM; $*: p<0.05$ compared to $W T, \#: p<0.05$ compared to Per $2 \mathrm{~m} / \mathrm{m}, \xi$ : $\mathrm{D}<0,05$ compared to Per2m/mDec1 / or Per $2 \mathrm{~m} / \mathrm{mDec} 2$ /. (B) Percentage of ihythmic animals during 21 davs in DD of wild-type (WT). Dec1/- Dec2 / (qrey line; per genotype $n=12$ ), Per $2 \mathrm{~m} / \mathrm{m}$ (red line; $n=9$ ), Per2m/mDec1 / (blue line; $n=12$ ), Per $2 \mathrm{~m} / \mathrm{mDec} 2$ / (areen line; $n=$ 11 ) and Per $2 m / m$ mec1/2 $/$ mice (black line; $n=5$ ). $81 \times 182 \mathrm{~mm}(600 \times 600 \mathrm{DPI})$ 
$\rightarrow$ ParanimDec1

A
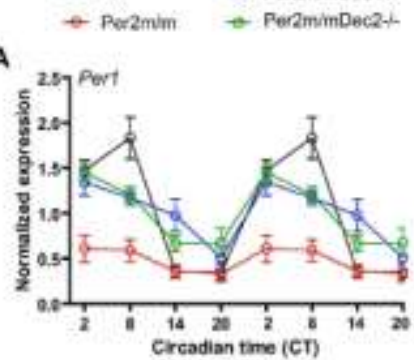

B
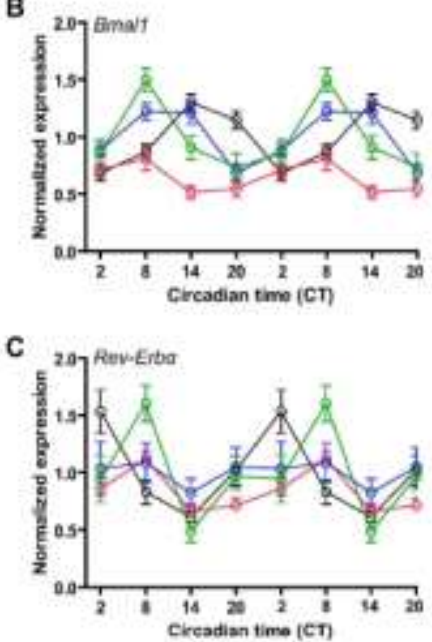

Bode et al. Figure 3

In situ hybridization (ISH) profiles of clock genes in the SCN. Normalized mRNA expression levels of (A) Per1, (B) Bmal1 and (C) Rev-Erbo in the SCN under constant darkness conditions of wild-type (WT; black line), Per $2 \mathrm{~m} / \mathrm{m}$ (red line), Per $2 \mathrm{~m} / \mathrm{mDec} 1$ / (blue line) and Per $2 \mathrm{~m} / \mathrm{mDec} 2$ / (qreen line) mice $(n=3)$, Values are double plotted and represented as mean $\pm 5 E M$

$$
107 \times 211 \mathrm{~mm}(600 \times 600 \mathrm{DPI})
$$

URL: http://mc.manuscriptcentral.com/lcbi E-mail: USA: michael.h.smolensky@uth.tmc.edu/ EUROPE: touitoughccr.jussieu.fr 

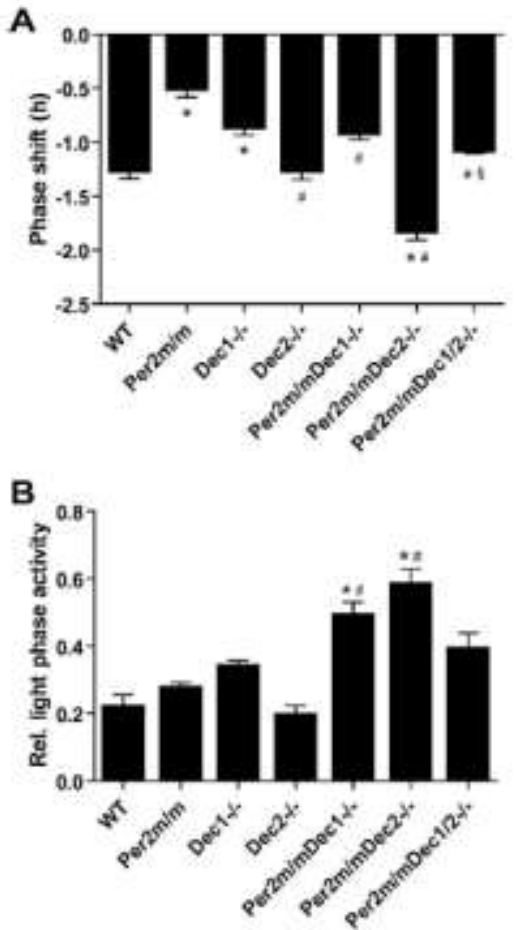

\section{Bode et al. Figure 4}

Phase delay and masking responses to noctumal light exposure. (A) Quantification of phase-delay responses after a $15 \mathrm{~min}$ liqht pulse at $2 \mathrm{~T}_{14} \mathrm{:}$ : WT $1,26 \pm 0.08 \mathrm{~h}(\mathrm{n}=8)$, Per2m/m $0.50 \pm 0.08 \mathrm{~h}$ ( $\mathrm{n}$

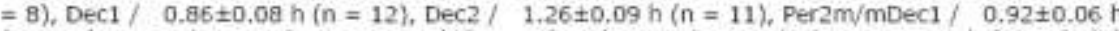
$(n=11)$, Per $2 \mathrm{~m} / \mathrm{mDec} 2 / 1.83 \pm 0.09 \mathrm{~h}(\mathrm{n}=11)$ and Per $2 \mathrm{~m} / \mathrm{mDec} 1 / 2 / 1.07 \pm 0.03 \mathrm{~h}(\mathrm{n}=5)$. (B) Relative light phase activity during light exposure (100 lux) at ZT14-ZT15: WT $0.22 \pm 0.03$ ( $n=12$ ), Per $2 \mathrm{~m} / \mathrm{m} 0.28 \pm 0,02(\mathrm{n}=10)$, Dec1 / $0.34 \pm 0.02(\mathrm{n}=6)$, Dec2 / $0.19 \pm 0.03$ ( $\mathrm{n}=6$ ), Per $2 \mathrm{~m} / \mathrm{mDec} 1 / 0.49 \pm 0.04(n=12)$, Per $2 \mathrm{~m} / \mathrm{mDec} 2 / 0.58 \pm 0.05(n=11)$ and Per $2 \mathrm{~m} / \mathrm{mDec} 1 / 2$ ) $0.39 \pm 0.05(n=5)$. Data are represented as mean \pm SEM; $*: p<0.05$ compared to $W T$, $\theta: p<$ 0.05 compared to Per $2 \mathrm{~m} / \mathrm{m}$, 5: $p<0.05$ compared to Per $2 \mathrm{~m} / \mathrm{mDec} 1$ / or Per $2 \mathrm{~m} / \mathrm{mDec} 2$. $76 \times 179 \mathrm{~mm}(600 \times 600 \mathrm{DPI})$

URL: http:I/mc.manuscriptcentral.com/lcbi E-mail: USA: michael.h,smolensky@uth.tmc.edu/ EUROPE: touitougccr.jussieu.fr 

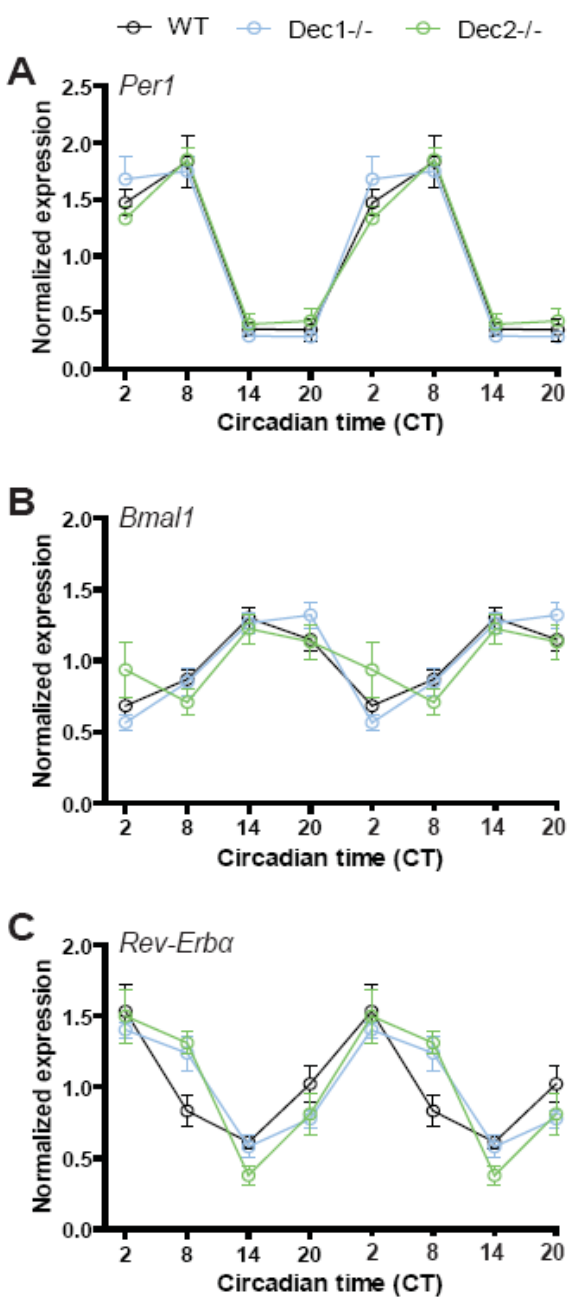

Bode et al. Suppl. Figure 1 

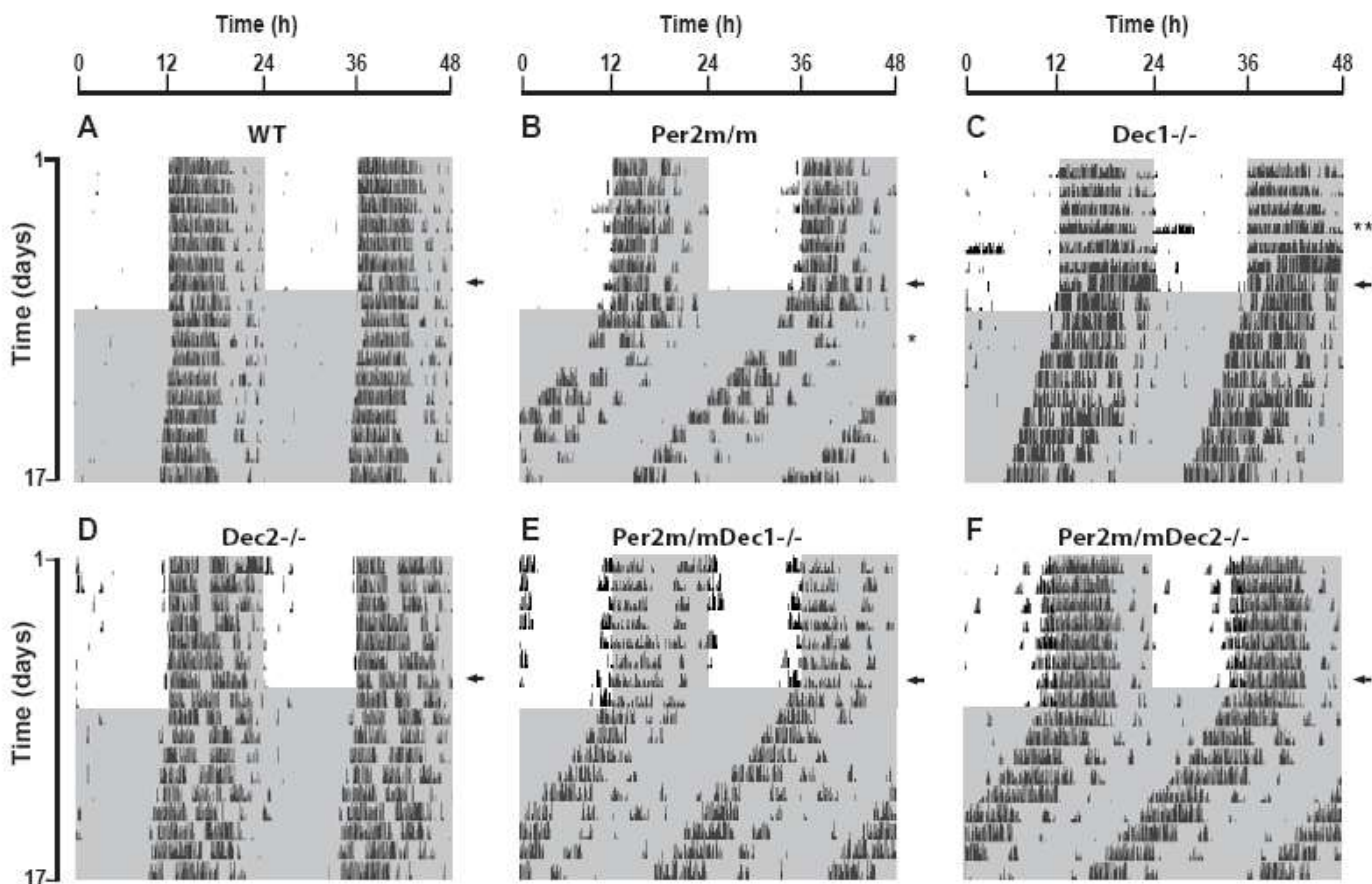

F Per $2 \mathrm{~m} / \mathrm{mDec} 2-/-$

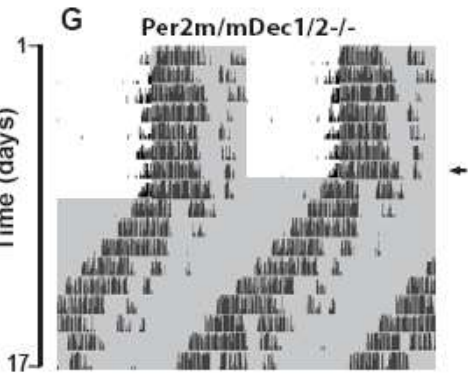

Bode et al. Suppl. Figure 2 
Supplenacuad Talik 1: Sasisticx of hetwioral paranetes

\begin{tabular}{|c|c|c|c|c|c|c|c|}
\hline Thed pataineter & $\begin{array}{l}\text { Geanespe } \\
\text { coapporben }\end{array}$ & Mriming & Dechos & Anclit & 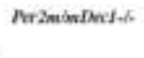 & Frizinivintat & Mrineinlacd/2K \\
\hline Oroat in 10 & 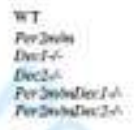 & $\cdots$ & 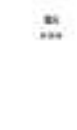 & $\begin{array}{l}m \\
w \\
w\end{array}$ & $\begin{array}{l}\cdots \\
\cdots\end{array}$ & $\begin{array}{l}\cdots \\
\cdots \\
m\end{array}$ & $\begin{array}{l}m \\
\cdots \\
\cdots \\
\cdots\end{array}$ \\
\hline Oreet rafieno in $L D$ & 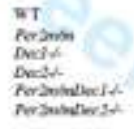 & $\cdots$ & $\therefore$ & $\begin{array}{l}* \\
a\end{array}$ & 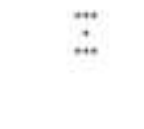 & $\begin{array}{l}\cdots \\
\cdots \\
m\end{array}$ & $\begin{array}{l}\cdots \\
\cdots \\
m\end{array}$ \\
\hline Period lenght in DO & 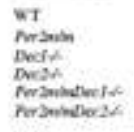 & $\ldots$ & $\cdots$ & $\begin{array}{l}a \\
\cdots \\
\cdots\end{array}$ & $\begin{array}{l}\cdots \\
\cdots \\
\cdots\end{array}$ & $\begin{array}{l}* \\
* \\
m\end{array}$ & $\begin{array}{l}m \\
m \\
m \\
m\end{array}$ \\
\hline Paotic exeving at 2714 & 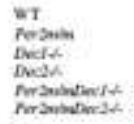 & $m$ & $\therefore$ & $\stackrel{4}{*}$ & a & $\begin{array}{l}\ldots \\
\ldots \\
\ldots\end{array}$ & $\begin{array}{l}z \\
z \\
z \\
z\end{array}$ \\
\hline Mading & 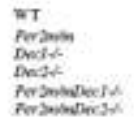 & a & $:$ & $\begin{array}{l}m \\
m \\
w\end{array}$ & 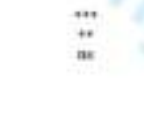 & $m$ & 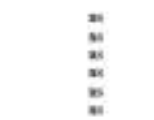 \\
\hline
\end{tabular}

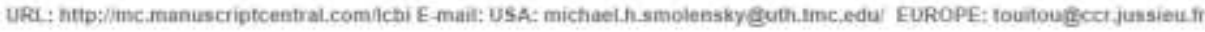




\subsection{Additional data}

\subsubsection{Phase delay responses and photic light suppression in Per1/Dec mutant mice}

Advanced activity onsets of Per2/Dec mutant mice in LD were correlated with impaired masking behaviour (see Results 3.2.). Per1/Dec mutant mice also showed this pre-dark activity, but to a lesser extent. To test if the observed advanced onsets might stem from either impaired masking or defective phase shifting capacities, we first examined photic phase delays in response to exposure to a $15 \mathrm{~min}$ light pulse at ZT15 (Aschoff type II protocol; Figure 15A-H). Single mutant mice showed no significant difference in phase shifting behaviour compared to wild-type animals, but Per1/Dec double mutants displayed increased, while Per1/Dec1/2 triple mutants showed decreased phase shifting responses (Figure $15 \mathrm{H}$ and statistics Table 1). These values did not correlate with the pre-dark activity phenotype of the different Per1/Dec mutant mice. Nevertheless, a synergistic interaction of Per1 and Dec1/2 in the regulation of phase delay resetting was observed.

We analyzed negative masking by applying a 100 lux light pulse for $1 \mathrm{~h}$ to stably entrained animals at ZT14-15. As expected, in wild-type mice the wheel-running activity was strongly suppressed during the $1 \mathrm{~h}$ light administration (Figure 15l). Single as well double mutants showed reduced activity comparable to that of wild-type controls (Figure $15 \mathrm{I}$ and statistics Table 1). In contrast, Per1/Dec triple mutant mice totally failed to suppress activity in response to light exposure (Figure $15 \mathrm{I}$ and statistics Table 1). Of note, the Per1/Dec triple mutant phenotype was not significantly different from that of Dec1/2 double mutants (data not shown). In summary neither masking nor phase resetting behaviour correlate with pre-dark activity in Per1/Dec mutant mice. Functional interactions of Per1 and Dec1/2 were observed for phase resetting, but not for masking (Results 3.1.). 

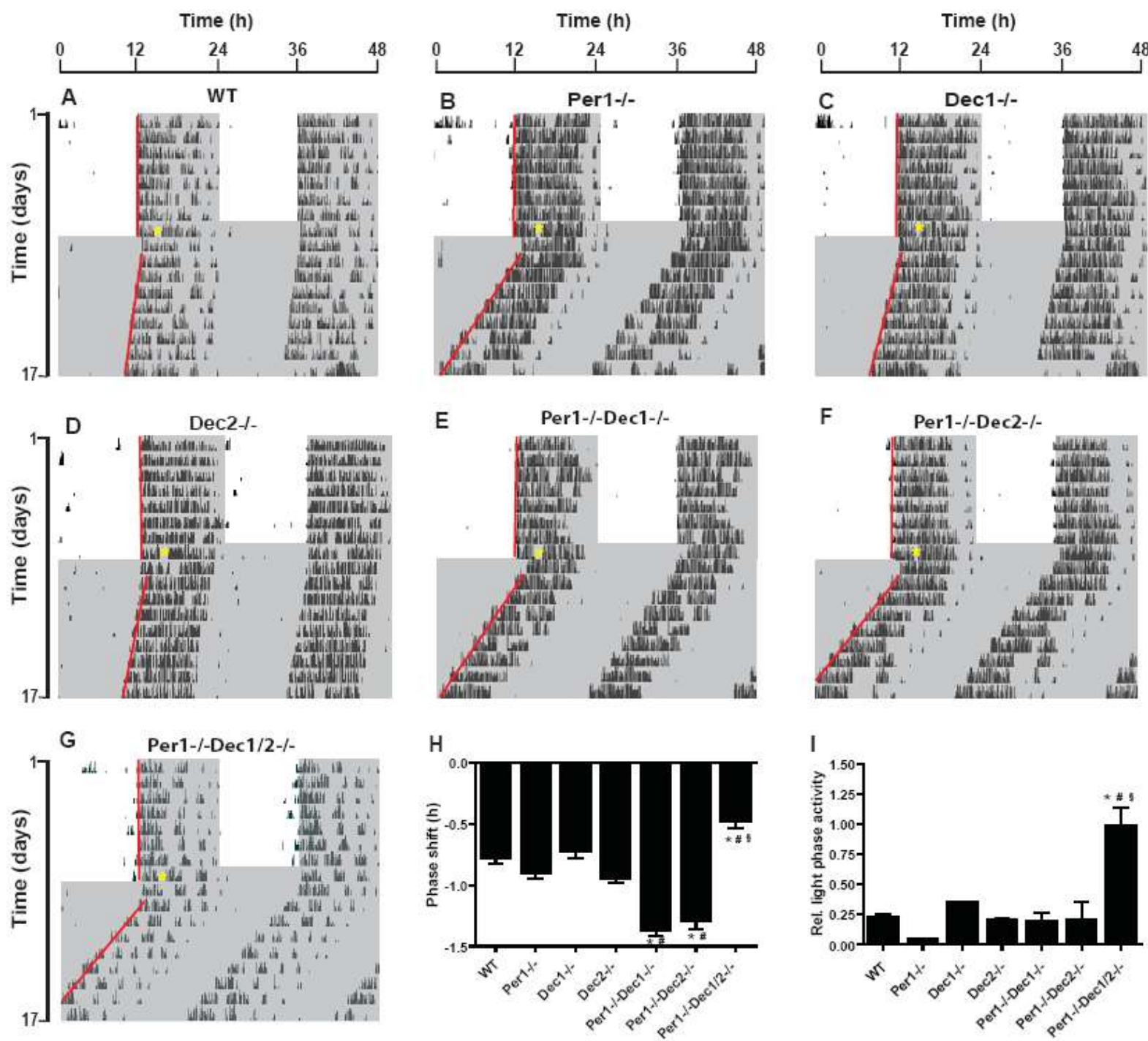

Figure 15. Photic phase resetting and negative masking. (A-G) Representative double plotted actograms of phase-delay response to 15 min light pulse treatments at ZT15 (yellow star) of (A) wild-type (WT), (B)

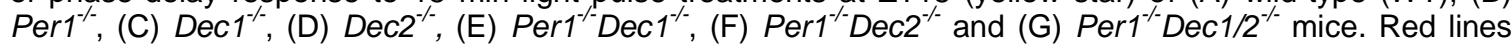
indicate regressions through the onsets of wheel-running activity for calculating activity phase shifts on the day after the light pulse administration. $(\mathrm{H})$ Quantification of phase-delay responses after a light pulse at

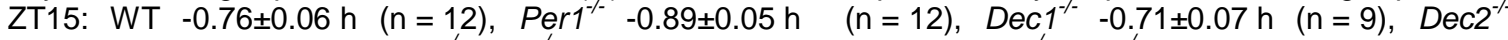

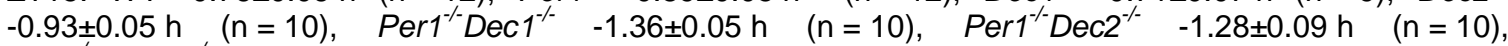
Perr $^{-1 /}$ Dec1/2/- $-0.46 \pm 0.07 \mathrm{~h}(\mathrm{n}=11)$. (I) Relative light phase activity during light exposure (100 lux) at ZT14ZT15: WT 0.22 $\pm 0.03(n=12)$, Per1 ${ }^{-1} 0.04 \pm 0.02(n=5)$, Dec1 ${ }^{-1} 0.34 \pm 0.02(n=6)$, Dec2 $2^{-1} 0.19 \pm 0.03(n=6)$,

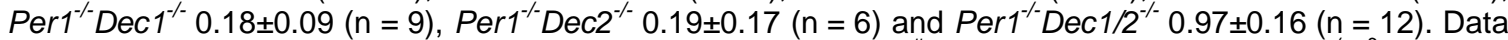
are represented as mean \pm SEM; ${ }^{*}: p<0.05$ compared to WT, ${ }^{\#}: p<0.05$ compared to Pert ${ }^{-/},{ }^{\$}: p<0.05$ compared to Per1 $1^{-1 /}$ Dec $1^{-1-}$ or Pert ${ }^{-1}$ Dec2 $2^{-1}$. 
Table 1: Statistics of behavioral tests

\begin{tabular}{|c|c|c|c|c|c|c|c|}
\hline $\begin{array}{l}\text { Tested } \\
\text { parameter }\end{array}$ & $\begin{array}{l}\text { Genotype } \\
\text { comparison }\end{array}$ & Per1-/- & Dec1-/- & Dec2-/- & Per1-/-Dec1-/- & Per1-/-Dec2-/- & $\begin{array}{c}\text { Per1-/- } \\
\text { Dec1/2-/- }\end{array}$ \\
\hline \multirow{6}{*}{$\begin{array}{l}\text { Phase } \\
\text { delay } \\
\text { (ZT15) }\end{array}$} & WT & ns & ns & ns & $* * *$ & $\star * *$ & * \\
\hline & Per1-/- & & ns & ns & $* * *$ & ** & $* * *$ \\
\hline & Dec1-/- & & & ns & $\star \star \star *$ & & ns \\
\hline & Dec2-/- & & & & & * & *** \\
\hline & Per1-/-Dec1-/- & & & & & ns & *** \\
\hline & Per1-/-Dec2-/- & & & & & & *** \\
\hline \multirow{6}{*}{ Masking } & WT & ns & ns & ns & ns & ns & $* * *$ \\
\hline & Per1-/- & & ns & ns & ns & ns & $* * *$ \\
\hline & Dec1-/- & & & ns & ns & & $\star \star *$ \\
\hline & Dec2-/- & & & & & ns & *** \\
\hline & Per1-/-Dec1-/- & & & & & ns & *** \\
\hline & Per1-/-Dec2-/- & & & & & & $\star \star * *$ \\
\hline
\end{tabular}

ns = not significant; ${ }^{*} p \leq 0.05 ;{ }^{* *} p \leq 0.01 ;{ }^{* * *} p \leq 0.001$

\subsubsection{Locomotor activity under constant light in Per2/Dec mutant mice}

Concluding the study on Per2-Dec interaction, we examined activity period length in LL (50 lux light intensity) in Per2/Dec single, double and triple mutants. Entrained mice were released into $L L$ and period length was determined by $x^{2}$ periodogram analysis over an interval of 7-10 consecutive days using ClockLab software. Following Aschoff's rule, period lengths were increased in LL compared to DD (Aschoff 1960; Aschoff 1979). The LL period of Per2 mutant mice was significantly shorter than that of wild-type animals. Per2/Dec double mutant mice did not show any significant period differences when compared to Per2 single mutants (Figure 16 and statistics Table 2) indicating that no functional interaction of Per2 and Dec1 or Dec2 was seen in LL. In contrast, Per2/Dec triple mutants showed a partial rescue of period length in LL compared to Per2 single or Per2/Dec double mutants (Figure 16 and statistics Table 2). 


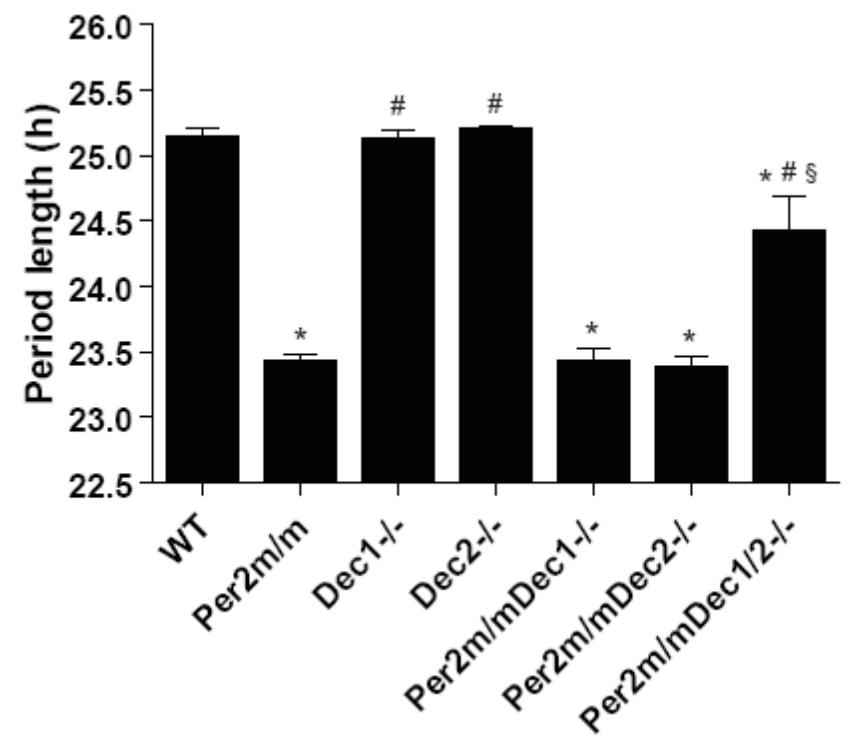

Figure 16. Locomotor activity in LL. Average free-running period length into constant light ( $L_{50}$ lux $)$ : WT $25.12 \pm 0.08 \mathrm{~h}(\mathrm{n}=12)$, Per2 $^{\mathrm{m} / \mathrm{m}}$ $23.40 \pm 0.09 \mathrm{~h} \quad(\mathrm{n}=14), \quad \operatorname{Dec} 1^{-1}$ $25.10 \pm 0.09 \mathrm{~h} \quad(\mathrm{n}=11), \quad \operatorname{Dec}^{-/}$ $25.18 \pm 0.06 \mathrm{~h} \quad(\mathrm{n}=12)$, Per2 ${ }^{m / m} \operatorname{Dec}^{-/-} 23.41 \pm 0.12 \mathrm{~h}(\mathrm{n}=5)$, Per2 ${ }^{m / m} D_{e c 2^{-/}} 23.36 \pm 0.10$ h $(n=5)$, Per2 ${ }^{m / m}$ Dec1/2 $\quad 24.41 \pm 0.29 \mathrm{~h}$ $(n=2)$. Data are represented as mean \pm SEM. ${ }^{*}: p<0.05$ compared to WT, ${ }^{\#}: p<0.05$ compared to Per2 ${ }^{m / m}, \quad s: p<0.05$ compared to Per2 ${ }^{m / m}$ Dec1 $^{-/-}$or Per2 ${ }^{m / m}$ Dec2 ${ }^{-/}$.

Table 2: Statistics of for LL period

\begin{tabular}{|c|c|c|c|c|c|c|c|}
\hline Paradigm & $\begin{array}{l}\text { Genotype } \\
\text { comparison }\end{array}$ & Per2m/m & Dec1-/- & Dec2-/- & $\begin{array}{c}\text { Per2m/m } \\
\text { Dec1-/- }\end{array}$ & $\begin{array}{l}\text { Per2m/m } \\
\text { Dec2-/- }\end{array}$ & $\begin{array}{l}\text { Per2m/m } \\
\text { Dec1/2-/- }\end{array}$ \\
\hline \multirow{6}{*}{$\begin{array}{l}\text { Period } \\
\text { length } \\
\text { in } L L\end{array}$} & WT & 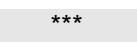 & ns & ns & *** & 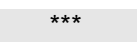 & * \\
\hline & Per2m/m & & *** & 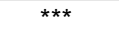 & ns & ns & *** \\
\hline & Dec1-/- & & & ns & *** & & * \\
\hline & Dec2-/- & & & & & 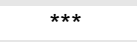 & * \\
\hline & Per2m/mDec1-/- & & & & & ns & $\star \star$ \\
\hline & Per2m/mDec2-/- & & & & & & $\star *$ \\
\hline
\end{tabular}

ns $=$ not significant $;{ }^{*} p \leq 0.05 ;{ }^{* *} p \leq 0.01 ;{ }^{* * *} p \leq 0.001$

Of note, all behavioural experiments (from Results 3.1., 3.2., 3.3.1 and 3.3.2.) were also performed on Dec1/2 double mutants to test the possibility that the observed Per/Dec triple mutant phenotype merely reflects the absence of both DECs. In each of the tests, triple mutants showed significant differences when compared to Dec1/2 animals (data not shown), except for the Per2Dec1/2 triple mutant masking phenotype. Phase delay shifting of triple mutants showed a trend towards a significant difference when compared to Dec1/2 mutant mice. 
Table 3 summarizes the findings from 3.1., 3.2., 3.3.1 and 3.3.2. with regard to Per-Dec interaction.

Table 3: Functional genetic interaction of Per1 or Per2 with Dec1 and/or Dec2.

\begin{tabular}{|l|ccc|ccc|}
\hline & \multicolumn{4}{|c|}{ Per1 } & \multicolumn{3}{c|}{ Per2 } \\
\cline { 2 - 7 } & Dec1 & Dec2 & Dec1/2 & Dec1 & Dec2 & Dec1/2 \\
\hline Onset in LD & + & n.i. & + & + & + & + \\
Onset variation in LD & + & n.i. & + & + & + & + \\
\hline Period length in DD & n.i. & n.i. & + & - & n.i. \\
Period length in LL & n.i. & n.i. & + & n.i. & n.i. & - \\
\hline Photic resetting & + & + & n.i. & n.i. & - & n.i. \\
\hline Masking & n.i. & n.i. & n.i. & + & + & n.i. \\
\hline
\end{tabular}

+: synergistic interaction; -: antagonistic interaction; n.i.: no genetic interaction

\subsubsection{Photic phase delays in Per single mutant mice}

The endogenous clock must be synchronized each day to stay in synchrony with the external light/dark cycle. This entrainment is based of daily resetting of the SCN clock by light. This photic phase resetting is hypothesized to depend on acute photic Per1 and Per2 induction in the SCN after light exposure during the night (see Introduction 1.4.). To understand the role of Per gene induction in clock resetting, we investigated phase delay resetting on the behavioural and molecular levels in Per1 and Per2 single mutants as well as in Dec1/2 double mutant mice using an Aschoff type II protocol. As expected, Per2 as well as Dec1/2 mutant mice showed decreased, and Per1 mutants normal phase shifting behaviour compared to wild-type mice (Figure 17 and statistics Table 4). This supports that PER2 and DECs have an essential function in phase delay resetting (Albrecht, Zheng et al. 2001; Rossner, Oster et al. 2008). 


\section{Time (h)}

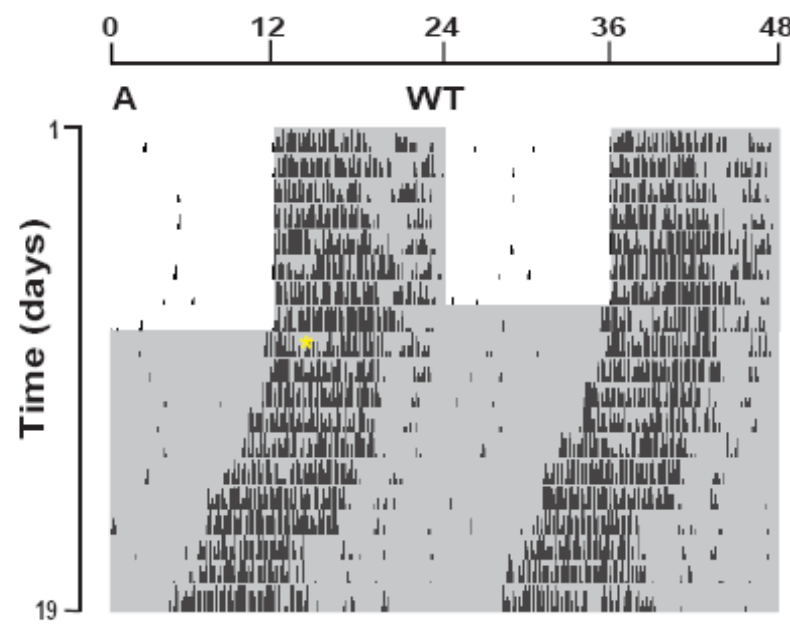

C
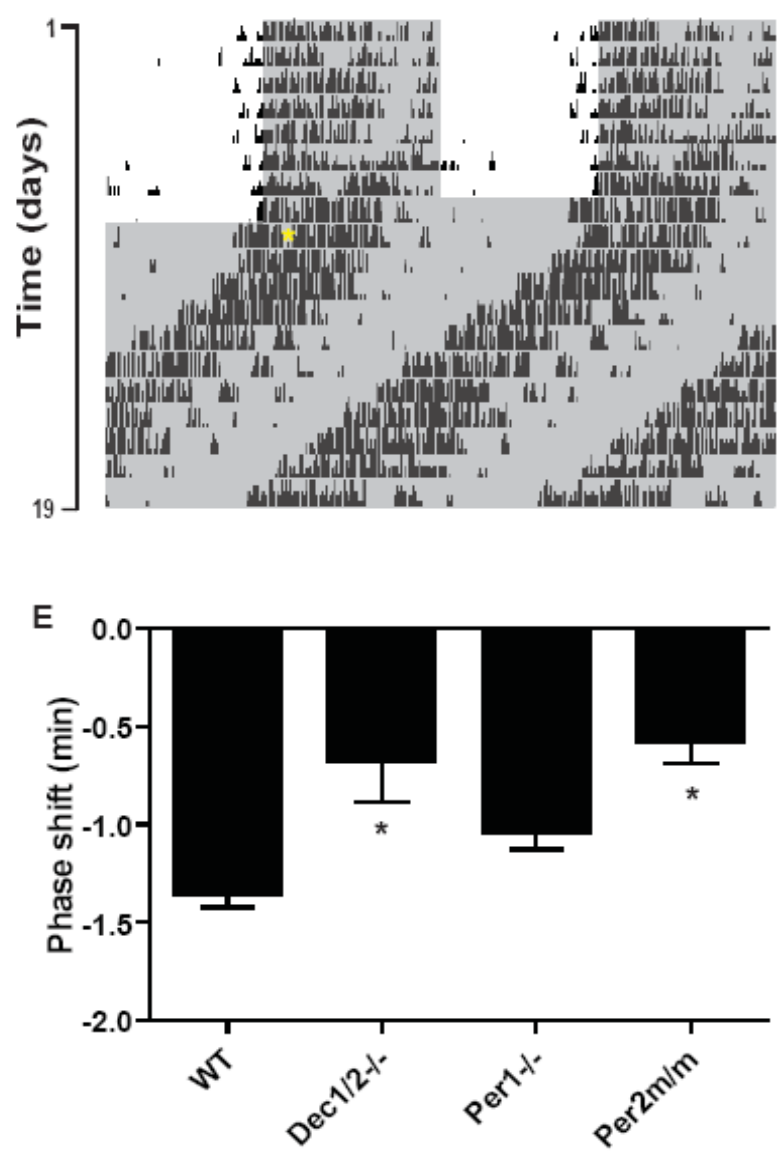

\section{Time (h)}
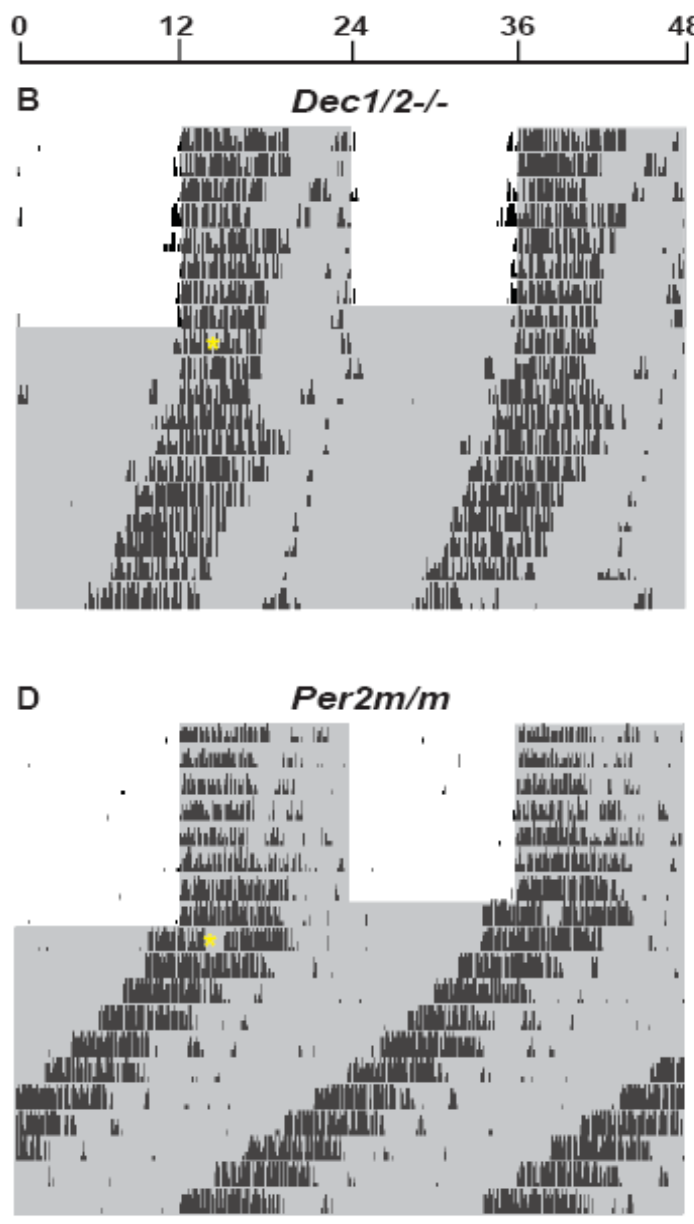

Figure 17. Phase shifts in response to light pulses at CT14. (A-D) Representative double plotted actograms of phase-delay responses after 15 min light pulse exposure at CT14 on the first day of constant darkness (yellow star) of $(A)$ wild-type (WT), (B) Dec1/2 ${ }^{-/}$, (C) Per1 ${ }^{-/}$and (D) Per2 ${ }^{\mathrm{m} / \mathrm{m}}$ mice. (E) Quantification of phase-delays: WT $-1.35 \pm 0.07 \mathrm{~h}$ $(\mathrm{n}=13), \quad$ Dec1/2 $2^{--}-0.67 \pm 0.21 \mathrm{~h} \quad(\mathrm{n}=15), \quad$ Per1 ${ }^{-1-}$ $-1.03 \pm 0.10 \mathrm{~h}(\mathrm{n}=16)$, Per2 $^{\mathrm{m} / \mathrm{m}}-0.57 \pm 0.12 \mathrm{~h}(\mathrm{n}=14)$. Data are represented as mean \pm SEM; * $p<0.05$ compared to WT. 


\begin{tabular}{|c|c|c|c|c|}
\hline Tested parameter & $\begin{array}{l}\text { Genotype } \\
\text { comparison }\end{array}$ & Dec1/2-/- & Per1-/- & Per2-/- \\
\hline \multirow{3}{*}{ Phase delay (CT14) } & WT & * & $\mathrm{ns}$ & ** \\
\hline & Dec1/2-/- & & ns & ns \\
\hline & Per1-/- & & & ns \\
\hline
\end{tabular}

ns $=$ not significant; ${ }^{*} p \leq 0.05 ;{ }^{* *} p \leq 0.01 ;{ }^{* * *} p \leq 0.001$

To compare behavioural and molecular light responses, photic Per1 and Per2 induction was measured in the SCN after a 15 min light pulse administration at CT14 on the first day in DD using in situ hybridization. Per mRNA levels in the SCN were quantified at $15 \mathrm{~min}, 30 \mathrm{~min}, 60 \mathrm{~min}, 120 \mathrm{~min}$ and $240 \mathrm{~min}$ after the beginning of the light treatment (Figure 18). As expected, in wild-type mice light pulses at CT14 induced Per1 as well Per2 expression in the SCN peaking $60 \mathrm{~min}$ and $120 \mathrm{~min}$, respectively, after the beginning of the light exposure (Figure 18 and Table 5) (Shigeyoshi, Taguchi et al. 1997; Yan and Silver 2002). Given that the Per mutants have non-functional PER1 or PER2 proteins, we did not investigate Per induction in the corresponding mutants. Per1 induction was reduced in Per2 mutant mice compared to wild-type mice (Figure 18; Table 5). We further investigated the Per induction in Dec1/2 mutants which show reduced behavioural resetting similar to that seen in Per2 mutant mutants (Figure 17E and statistics Table 4) (Rossner, Oster et al. 2008). In contrast to Per2 mutant mice, Dec1/2 mutant animals showed increased Per1 induction compared to wild-type animals (Figure 18 and Table 5). Additionally, Dec deficiency had an influence on Per2 induction as Per2 peak expression was advanced by $90 \mathrm{~min}$ and overall induction was decreased in the Dec1/2 mutants (Figure 18 and Table 5). Per1 mutants showed increased Per2 mRNA levels at the peak time compared to wild-type animals (Figure 18 and Table 5). 
Per1 expression

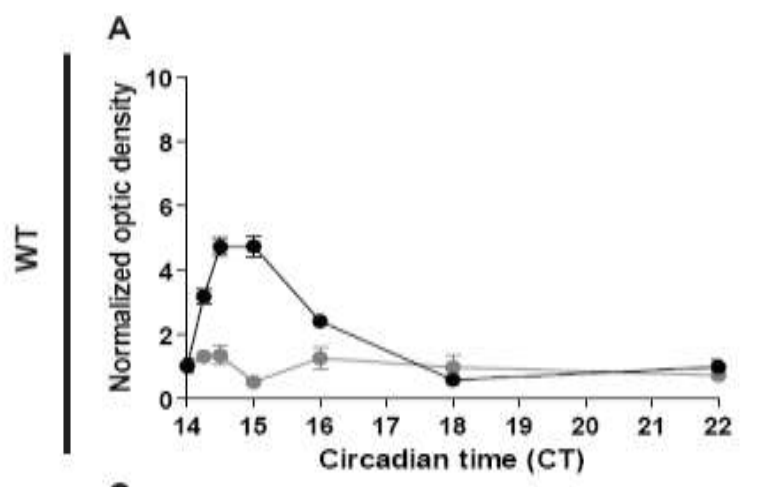

C

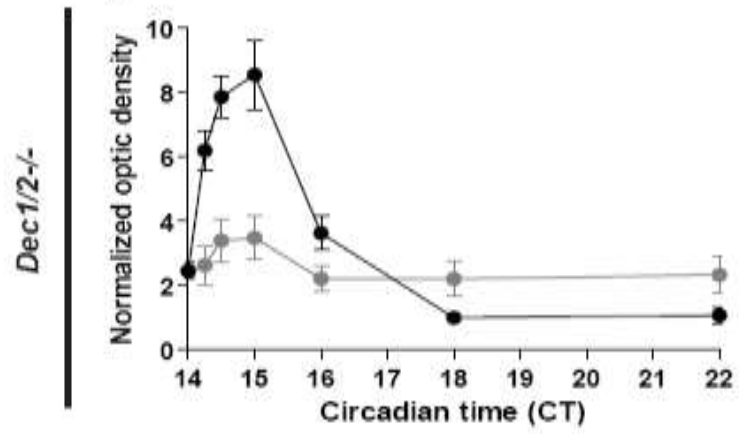

n.a.
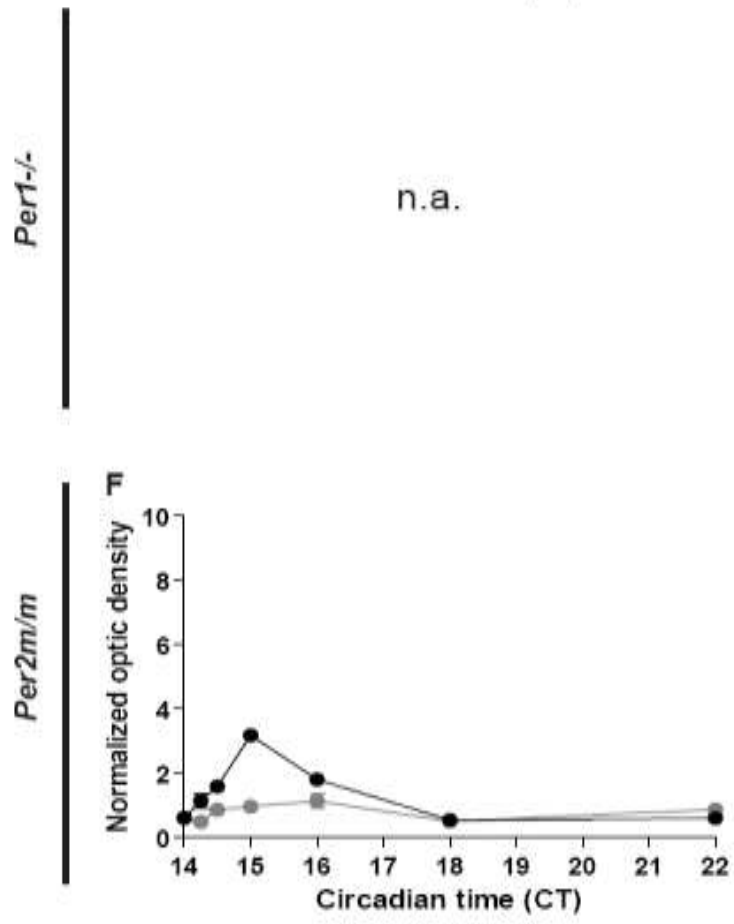

\section{Per2 expression}

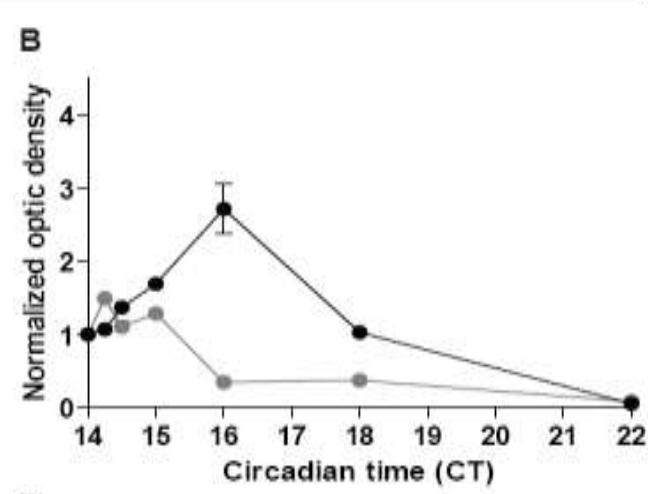

D
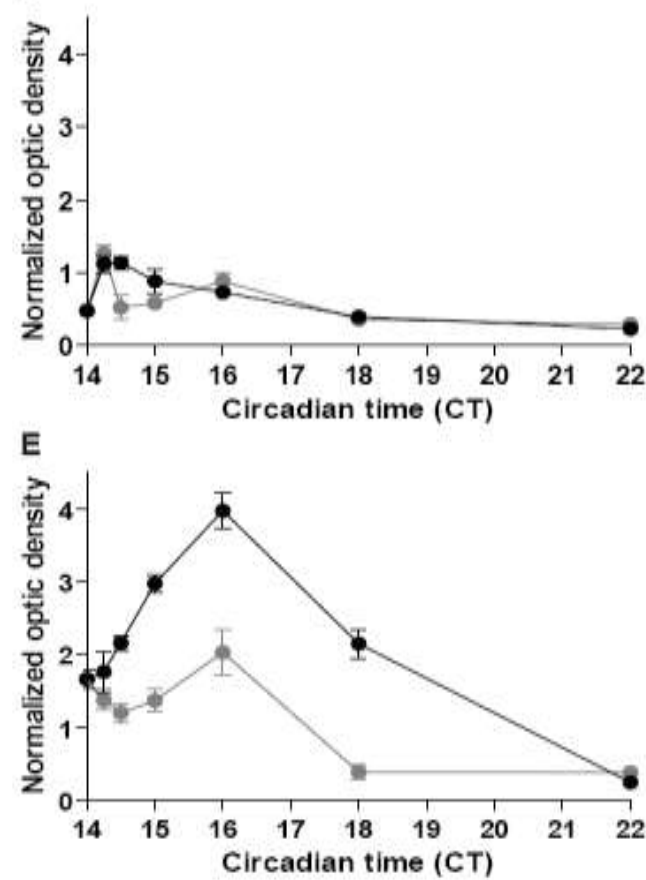

Figure 18. In situ hybridization (ISH) of photic Per gene inductions in the SCN. Normalized mRNA expression levels of Per1 (left column; A, C and F) and Per2 (right column; B, D and E) in the SCN under constant darkness (grey line) or with a 15 min light exposure at CT14 on the first day in DD (black line) in wild-type (WT; upper line), Dec1/2/- (second line), Per1 ${ }^{-/-}$(third line) and Per2 ${ }^{\mathrm{m} / \mathrm{m}}$ (lower line) mice $(\mathrm{n}=3)$. Values are represented as mean \pm SEM. n.a.: not analyzed 
Table 5: Photic Per induction in the SCN after a phase delay light pulse

\begin{tabular}{|l|cc|cc|}
\hline & \multicolumn{2}{|c|}{ photic Per1 induction } & \multicolumn{2}{c|}{ photic Per2 induction } \\
\cline { 2 - 5 } & $\begin{array}{c}\text { peak after light } \\
\text { pulse }\end{array}$ & peak expression & $\begin{array}{c}\text { peak after light } \\
\text { pulse }\end{array}$ & peak expression \\
\hline WT & $60 \mathrm{~min}$ & $4.72 \pm 0.33$ & $120 \mathrm{~min}$ & $2.71 \pm 0.33$ \\
Dec1/2-/- & $60 \mathrm{~min}$ & $8.56 \pm 1.08 * \#$ & $30 \mathrm{~min}$ & $1.14 \pm 0.09 * \#$ \\
Per1-/- & n.a. & n.a. & $120 \mathrm{~min}$ & $3.97 \pm 0.23$ * \\
Per2m/m & $60 \mathrm{~min}$ & $1.81 \pm 0.14 *$ & n.a. & n.a. \\
\hline
\end{tabular}

n.a. = not analyzed; * significant to wild-type ${ }^{*}$ significant to Per mutant 


\section{Chapter 4: Discussion and Perspective}

The molecular components of the Drosophila and the mammalian TTLs are largely conserved and their functions are very similar (Bell-Pedersen, Cassone et al. 2005; Looby and Loudon 2005). However, gene duplications resulted in multiple copies of some fly clock genes in mammals (e.g. Per1-3, Dec1-2). The two mammalian Dec genes have one ortholog in the Drosophila circadian clock-clockwork orange (cwo)-with similar transcriptional regulatory functions. For the fruit fly, it was postulated that PER and CWO have a synergistic function in the circadian timekeeping system (Kadener, Stoleru et al. 2007). Hence, we hypothesised a synergism of action for $\operatorname{Per}(1,2)-\operatorname{Dec}(1,2)$ in the regulation of the mammalian circadian clock. To test this hypothesis, we analysed circadian behaviour and SCN clock gene expression rhythms of homozygous Per1/Dec and Per2/Dec double and triple mutant mice. Per1-Dec and Per2-Dec interactions are discussed separately in the attached manuscripts (Results 3.1. and 3.2.). Therefore, in this chapter I will focus on combining these findings.

\subsection{Synergistic Per1-Dec and Per2-Dec interaction in photic entrainment}

The pre-dark activities of $\operatorname{Per}(1,2) / D e c$ double and triple mutants show a $\operatorname{Dec}$ gene dependent increase compared to the corresponding $\operatorname{Per}(1,2)$ single mutant phenotype (Per1/Dec1 1.9 fold, Per2/Dec1 1.9 fold; Per2/Dec2 2.0 fold; Per1/Dec1/2 2.8 fold, Per2/Dec1/2 2.7 fold advance of onset activity to the corresponding Per single mutants). These data suggest that the strength of Per1-Dec interaction in photic entrainment is similar to that of Per2 and Dec1/2. These interactions appear to be necessary for efficient synchronization of the activity phase to the external photic environment, and are synergistic (Results 3.1. and 3.2.). Whether they are based on physical association of the corresponding proteins, or rather reflect a functional genetic interaction, e.g. via coregulatory effects on target gene activation, remains to be shown. Nevertheless, combined Per-Dec deficiency results not only in changes on the transcription level of clock genes in constant darkness (Results 3.1., 3.2.), but perhaps already in LD. Thus, analysis of clock gene expression under entrained conditions might provide evidence for 
clock involvement in the pre-dark phenotype, especially during the second half of the light phase. In line with this, it was suggested for the pre-dark phenotype of Per2 single mutants (Results 3.2.) that advanced Bmal1 expression in the SCN might underlie the observed phase advance in activity in LD (Oster, Yasui et al. 2002). We show similarly advanced Bmal1 expression in Per2 and Per2/Dec double mutants in DD (Results 3.2.), supporting the view that an advanced Bmal1 expression rhythm might cause pre-dark activity in the double and triple mutants.

For the Per2-Dec1 and Per2-Dec2 interactions correlations between onset activity and masking during the early dark phase (Figure 15I, Table 3) were shown. In contrast, the pre-dark activity of Per1/Dec mutants does not correlate neither to negative masking nor to phase delay phenotypes (Figure 15). Another possible reason might be disrupted sleep behaviour, as discussed in Results 3.1. Therefore, sleep behaviour in these double and triple mutant mice remains to be investigated.

\subsection{Compensation effects cause partial rescue in free-run}

Under photic entrainment conditions Per-Dec interactions are highly synergistic. In contrast, in the absence of the Zeitgeber light (i.e. DD or LL) Per-Dec interaction properties change (Table 3). The Per1-Dec interactions stay synergistic with functional $D e c$ redundancy in free-run. The Per2-Dec interactions, however, change to antagonistic functionality and a partial rescue of the Per2 phenotype occurs (period length, rhythmicity and clock gene expression in the SCN) in DD (Results 3.2.), suggesting a conserved Dec redundancy and postulating partial compensation by the corresponding PER1/DEC(1 or 2) protein complexes (Results 3.2.). Similar mechanisms were postulated by Oster et al. for Per/Cry interactions (Oster, Baeriswyl et al. 2003). Supporting this view, it was previously shown that the remaining Dec can compensate for deficiency of the corresponding other Dec gene in vitro and in vivo by increased expression levels (Grechez-Cassiau, Panda et al. 2004; Nakashima, Kawamoto et al. 2008; Liu, Sato et al. 2010). However, such compensation seems not to be sufficient to rescue the Per1 phenotype under constant conditions (Results 3.1.). We suggest a model in which PER/DEC compensation shares some similarities (Figure 19) with the suggested PER/CRY compensation mechanism (Oster, Baeriswyl et al. 2003). According to this model, during the first days of darkness the compensation effect is strong, because the animals do not loose rhythmicity immediately (Results 3.2.). After several days, the 
compensation capacity falls below a hypothetic threshold which is critical for normal clock function (Figure 19) - of note, in contrast to Per2 and Per2/Dec1/2 mutants only some Per2/Dec double mutants fall below the threshold and, hence, become arrhythmic. Efficient compensation of Per/Dec deficiency is restricted to PER1(CRY)/DEC(1 or 2) resulting in modified - but rescued - clock gene expression in the SCN (Results 3.2.) and perhaps also in peripheral tissues. Considering the behavioural phenotype of Per2/Dec1/2 mutant mice, we would expect a non-rescued clock gene expression similar to that seen in Per2 mutants, in case that PER1(CRY) would not be sufficient to compensate Per2/Dec1/2 deficiency in vivo.

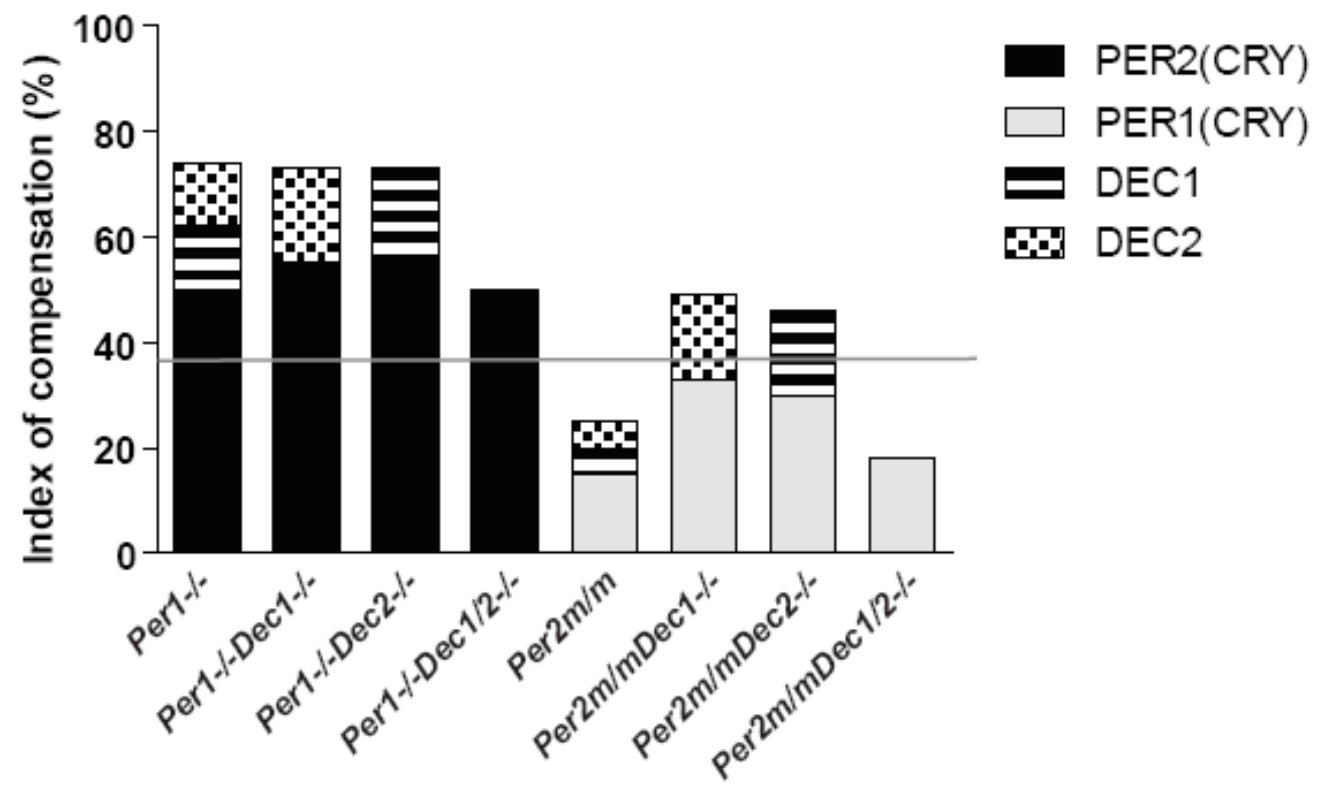

Figure 19. Compensation model. The model illustrates the compensation of Per/Dec deficiency with the indicated proteins after several days in DD. Black shows PER2(CRY), grey the PER1(CRY), black-white striped the DEC1 and black dotted the DEC2 proteins which mediate the corresponding compensation. The grey line represents the threshold for sufficient compensation to drive the rhythm of the circadian clock. The compensation effect for the Per1/Dec deficiency is not sufficient to increase the published Per1 deficiency compensation. In contrast, the compensation of Per2/Dec(1or2) deficiency is sufficient by increasing the compensation effect compared to Per2 deficiency accompanied with exceed of the threshold for sufficient clock function, but PER1(CRY) compensation of the Per2/Dec1/2 deficiency falls below the threshold.

Of note, Per2/Dec1/2 triple mutants show a partial rescue of period length in LL (Figure 16) similar to the Per2/Dec double mutant phenotype in DD (Results 3.2.). The restoration of period length in $L L$ is most likely based on functional Dec redundancy. It would be interesting to test if the observed rescue in LL is also present at the molecular level, as it was shown for DD (Results 3.2.). Taken together, synergistic Per1-Dec interactions are present during photic entrainment and free-run, together with Dec 
redundancy. In contrast, Per2-Dec synergism is restricted to photic entrainment and becomes antagonistic in free-run. This might be based on a disrupted compensation effect of PER1(CRY)/DEC (Figure 19). The stability of the clock depends on Per1 and Per2 function (Results 3.1., 3.2.) (Zheng, Larkin et al. 1999; Zheng, Albrecht et al. 2001) and is modified by the redundant Decs based on their interaction with the Pers.

\subsection{Bimodular transcriptional function of DEC in the mammalian pacemaker}

On the molecular level, we observed DEC redundancy and interactivity with PER in the SCN. We cannot exclude the possibility that the DECs also have regulatory functions independent of the PERs on clock gene expression, e.g. in the periphery. For example, in a cell-based luciferase assay, DECs alone activate Bmal1 expression, but PER1 potentiates their function as activators. We postulate a daytime dependent and bidirectional (activator and repressor) regulatory function of Per1-Dec interaction on E-box containing clock genes (see model in Figure 20), and a role as activators (direct or indirect) on Bmal1 transcription in the circadian pacemaker (Results 3.1.). The model in Figure 20 illustrates the daytime dependent bimodular regulatory functionality of Per1-Dec interaction on E-box containing genes during entrained conditions in the SCN. During the day, the CLOCK/BMAL1 complex activates the transcription by binding to E-box containing promoters (Hogenesch, Gu et al. 1998; Hamilton and Kay 2008). The observed inhibitory effect of PER1/DEC1/2 on Rev-Erba transcription might result from direct interaction with the CLOCK/BMAL1 complex. At the beginning of the night, the DECs might activate the expression of Rev-Erba and Per2 by direct binding to the E-box elements of their promoters. Limitations for this model are that PER1/2 as well as DEC1/2 proteins are rhythmically present in the SCN with a decreased level during the night and an increase in the light phase. Testing protein-protein interaction studies of PER1/DEC1/2 to BMAL1 (supporting the day function) and analyzing the protein interaction abilities on the promoter regions of Rev-Erba and Per2 by ChIP-Seq (supporting the night function) during the day might clarify this issue. 


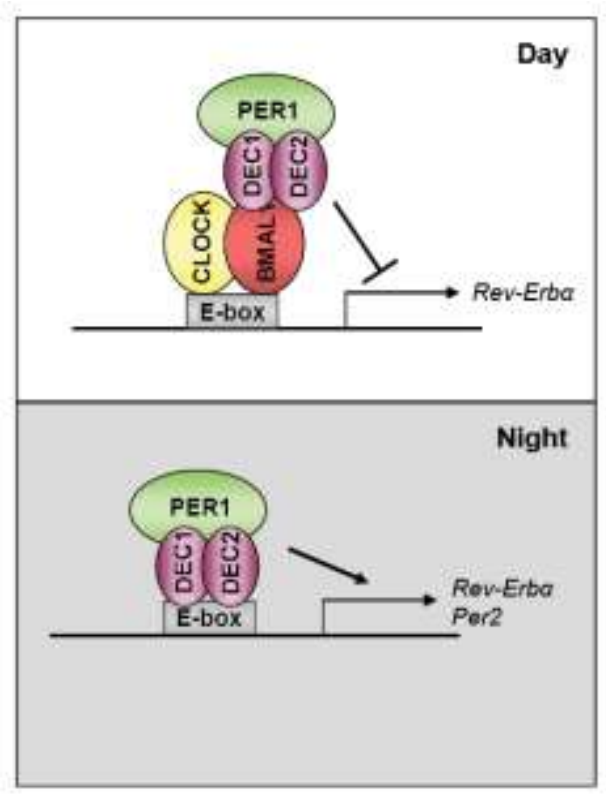

Figure 20. Daytime dependent bimodular PER1DEC1/2 function in the SCN. During the light phase (Day; upper part) the synergistic PER1 and DEC1/2 complexes inhibit CLOCK/BMAL1mediated transcription of E-box regulated genes such as Rev-Erba by direct interaction with CLOCK/BMAL1. During dark phase (Night; lower part) CLOCK/BMAL1 complexes are not present in the dark phase. Thus, PER1/DEC1/2 functions might be modified by the ability to direct binding to E-box elements resulting in activation of transcription of targets such as Rev-Erba and Per2.

Of note, for our model we assume that PER and DEC proteins physically interact, but this has yet to be proven. Further, we cannot exclude that the functionality of the Per-Dec interaction in the periphery might be different to the SCN (Figure 20). Overall, the molecular changes on clock gene expression in the SCN are represented in the behavioural phenotypes under constant darkness (Results 3.1., 3.2.).

\subsection{Per2 expression is essential for phase delay resetting}

The synchronization of the endogenous clock of the animal to the environmental light/dark cycle is based on a daily shift of the TTL in the SCN (see Introduction 1.4.). It was shown that the expression levels of Per1 and Per2 are induced in the SCN after a light pulse during the night (Shigeyoshi, Taguchi et al. 1997; Yan, Takekida et al. 1999; Yan and Silver 2002), and Per1 and Per2 single mutants display impaired phase shifting behaviour for phase advance and delay responses, respectively (Albrecht, Zheng et al. 2001). We focused on the phase delay response to determine the role of Per induction in photic resetting. The reduced behavioural resetting responses of Per2 and Dec1/2 mutant mice support the hypothesis that Per2 and Decs are involved in phase delay resetting (Figure 17) (Albrecht, Zheng et al. 2001; Rossner, Oster et al. 2008). Considering the reduced resetting behaviour of Dec1/2 double mutants and the blunted Per2 induction but increased Per1 induction - in the SCN of these animals, our data suggest that the 
Per2 induction was too weak to mediate an efficient phase shift in the TTL. This proposes an essential Per2/PER2 function in phase delay resetting on the molecular and behavioural levels. Therefore, we suggest a model in which photic Per2 induction efficiently delays the circadian system by exceeding a Per2 mRNA threshold (Figure 21). In wild-type mice, sufficient additional PER2 protein is produced to extend the inhibition of the CLOCK/BMAL1 complex. This prolonged repression results in a delay of the molecular oscillator, resulting in the observed behavioural phase delay response on the next day.
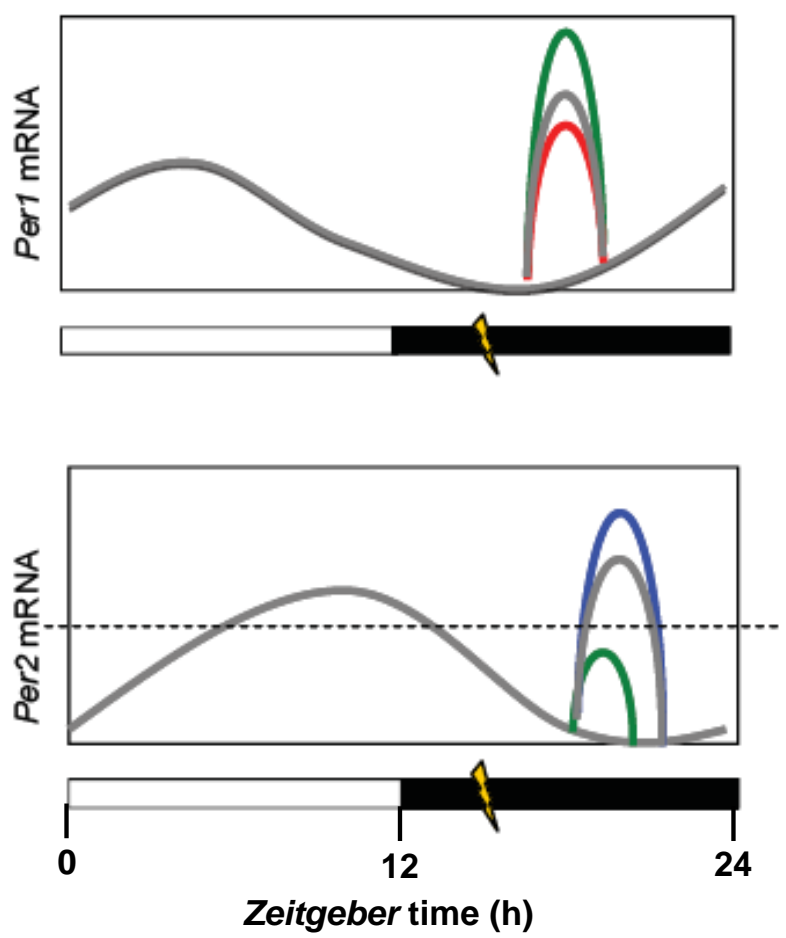

Figure 21. Model of photic Per induction in the SCN during phase delay resetting. The model shows the mRNA level of Per1 (upper panel) and Per2 (lower panel) after a light exposure in the first half of the night of wild-type (grey), Dec1/2/- (green), Per1 ${ }^{-/}$ (blue) and Per2 ${ }^{m / m}$ (red) mice. The white and black bars indicate the day and night, respectively, and the yellow flash the light pulse. The dashed line indicates the threshold of Per2 induction sufficient to mediate a phase delay shift.

\subsection{Potentiated PER1/DEC1 compensation elevates Per1 expression efficiently for phase delay resetting}

For phase delay responses we observed an antagonistic Per2-Dec2 interaction, but no functional interaction between Per2 and Dec1 (Results 3.2.; Table 3). The strong phase delay response of Per2/Dec2 mutant mice (Results 3.2.) might be explained by a similar PER1/DEC1 compensation mechanism as postulated for free-run. Of note, both of these genes are light inducible (Shigeyoshi, Taguchi et al. 1997; Yan, Takekida et al. 1999; Honma, Kawamoto et al. 2002; Yan and Silver 2002), which might potentiate the 
compensation effect resulting in a strong phase delay response. This hypothesis could be tested by molecular analysis of Per1 and Dec1 expression following light exposure. If confirmed, it would suggest that photic Per1 induction is potentiated by this compensation effect and is able to efficiently mediate a phase delay shift (Figure 22) by extended repression of the CLOCK/BMAL1 complex. According to this hypothesis, Per2/Dec1 and Per2/Dec1/2 mutants, which show weak phase delay responses (Results 3.2.), are not able to produce enough Per1 mRNA following light exposure because the light-inducible Dec1 gene is absent in these mutants.

Reduced phase delay resetting in Per1/Dec1/2 mutants (Figure 15H) might reflect the reduced phase delay response already observed in Dec1/2 mutants (data not shown) (Rossner, Oster et al. 2008). Alternatively, it may be caused by grossly impaired overall light reactivity (97\% activity) as measured in negative masking (Figure 15I). In contrast, impaired light sensitivity in Per2/Dec mutants appears not to be strong enough to significantly influence resetting behaviour, suggesting that sufficient phase shifting requires a defined light input (intensity and/or duration), which is not reached in Per1/Dec1/2, but is reached in Per2/Dec mutants. Photic Per2 induction in Per1/Dec double mutants might be stronger than in the Per1 single mutants (Figure 21), causing the observed stronger phase delay effect (Figure 15H) and postulating that Per1-Dec1 and Per1-Dec2 synergisms are involved in the regulation of Per2 induction by light. To test this hypothesis, photic Per2 induction should be determined in the SCN. Figure 22 shows the suggested photic Per induction of these mutants.
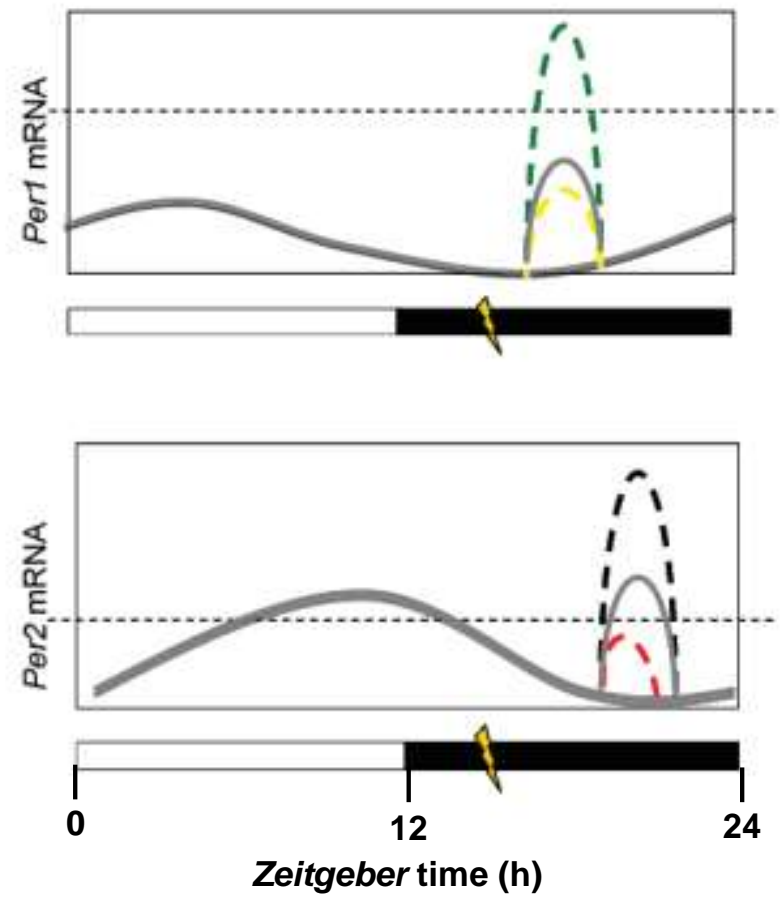

Figure 22. Model of photic Per induction in the SCN during phase delay resetting of the Per/Dec mutants. The model shows the hypothetical mRNA level (dashed line) of Per1 (upper panel) and Per2 (lower panel) induction after a light exposure in the first half of the night for wild-type (grey), Per2 $^{m / m} \operatorname{Dec2}^{-/} \quad$ (green, dashed), Per2 ${ }^{m / m}$ Dec1 ${ }^{-/-}, \quad$ Per2 $2^{m / m}$ Dec1/2 ${ }^{-/-} \quad$ (yellow dashed), Per1 ${ }^{-/}$Dec1 ${ }^{-/}$, Per1 $1^{-/}$Dec2 ${ }^{-/}$(black dashed) and Per1 ${ }^{-/}$Dec1/2\% (red dashed) mice. The white and black bars indicate day and night, respectively, and the yellow flashes represent the light pulse. The horizontal dashed line indicates the threshold of Per induction sufficient to mediate a phase delay shift. 
In summary, phase delay responses are primarily based on Per2 induction. It seems that the DECs, as well synergistic Per1-Dec interactions, are involved in regulating photic Per2 induction in the SCN. Of note, if Per1 induction is extended, e.g. by photic potentiation of a compensation effect as in Per2/Dec2 mutants, Per1/PER1 levels become sufficient to mediate a phase delay shift. Overall, higher levels of PER1 are necessary compared to PER2 in order to efficiently delay the TTL. Therefore, PER2 is the ratelimiting factor for phase delays of the clock.

\subsection{Conclusion and Perspective}

It was postulated that PER and CWO synergize and inhibit CLK-mediated activation in the Drosophila TTL (Kadener, Stoleru et al. 2007), but it has also been shown that CWO can activate clock gene expression (Richier, Rodriguez-Lanetty et al. 2008). During evolution, the core molecular components of the circadian oscillator have been largely conserved between diverse organisms such as Drosophila and mice (Looby and Loudon 2005). Gene duplication has resulted in multiple copies of clock genes (e.g. Per1-3, Cry1-2, Bmal1-2, Clock and NPAS2) with functional compensation properties (e.g. PER/CRY compensation (Oster, Baeriswyl et al. 2003), functional redundancy (e.g. Cry (van der Horst, Muijtjens et al. 1999) and diversity of circadian regulatory mechanisms in multiple tissues (e.g. NPAS2 is the ortholog of CLOCK in peripheral tissues). These gene duplications should result in higher robustness and adjustment. Thus, gene duplications of core clock components have increased the fitness of the circadian system during evolution. In this study, we found for the fly's per-cwo synergism (Kadener, Stoleru et al. 2007) an analog Per-Dec interaction in mammals, indicating a conservation of these mechanisms during evolution. The fact that the Dec single and double mutants show mild or no phenotypes and only in the additional absence of Per, the role of the Decs become unveiled, suggests that the DECs have fine-tuning roles in the circadian system.

The observed pre-dark activity indicates possible impaired sleep behaviour which might be used for modelling ASPS in mice, and offers the opportunity to investigate physiological interactions between sleep and the circadian system and to test treatments (e.g. melatonin administration) of ASPS to adjust sleep phase to the external environment. 
In addition, the Decs are functionally redundant in the circadian system in vivo - and we are the first group to show that the DECs together with PER1 positively regulate Bmal1 transcription in the SCN. Furthermore, Per1-Dec interaction is time-dependent and bimodular with respect to its influence on E-box containing clock gene transcription (Figure 20), suggesting that other components of the TTL might also show bimodular functionality in the mammalian TTL in the SCN and in peripheral tissues. Further characterization of the Per-Dec interaction will provide new insights into the entrainment and stabilizing mechanism of the mammalian circadian system in vivo. 


\section{References}

Akashi, M. and T. Takumi (2005). "The orphan nuclear receptor RORalpha regulates circadian transcription of the mammalian core-clock Bmal1." Nat Struct Mol Biol 12(5): 441-448.

Akashi, M., Y. Tsuchiya, et al. (2002). "Control of intracellular dynamics of mammalian period proteins by casein kinase I epsilon (CKlepsilon) and CKIdelta in cultured cells." Mol Cell Biol 22(6): 1693-1703.

Akhtar, R. A., A. B. Reddy, et al. (2002). "Circadian cycling of the mouse liver transcriptome, as revealed by cDNA microarray, is driven by the suprachiasmatic nucleus." Curr Biol 12(7): 540-550.

Albrecht, U., Z. S. Sun, et al. (1997). "A differential response of two putative mammalian circadian regulators, mper1 and mper2, to light." Cell 91(7): 1055-1064.

Albrecht, U., B. Zheng, et al. (2001). "MPer1 and mper2 are essential for normal resetting of the circadian clock." J Biol Rhythms 16(2): 100-104.

Albus, H., M. J. Vansteensel, et al. (2005). "A GABAergic mechanism is necessary for coupling dissociable ventral and dorsal regional oscillators within the circadian clock." Curr Biol 15(10): 886-893.

Allen, R. P., D. Picchietti, et al. (2003). "Restless legs syndrome: diagnostic criteria, special considerations, and epidemiology. A report from the restless legs syndrome diagnosis and epidemiology workshop at the National Institutes of Health." Sleep Med 4(2): 101-119.

Alvarez, J. D. and A. Sehgal (2002). "Circadian rhythms: finer clock control." Nature 419(6909): 798-799.

Ando, H., H. Yanagihara, et al. (2005). "Rhythmic messenger ribonucleic acid expression of clock genes and adipocytokines in mouse visceral adipose tissue." Endocrinology 146(12): 5631-5636.

Antle, M. C. and R. Silver (2005). "Orchestrating time: arrangements of the brain circadian clock." Trends Neurosci 28(3): 145-151.

Antle, M. C., V. M. Smith, et al. (2009). "Physiological responses of the circadian clock to acute light exposure at night." Rev Endocr Metab Disord 10(4): 279-291.

Aschoff, J. (1960). "Exogenous and endogenous components in circadian rhythms." Cold Spring Harb Symp Quant Biol 25: 11-28.

Aschoff, J. (1979). "Circadian rhythms: influences of internal and external factors on the period measured in constant conditions." Z Tierpsychol 49(3): 225-249.

Atkinson, H. C., S. A. Wood, et al. (2006). "Diurnal variation in the responsiveness of the hypothalamic-pituitary-adrenal axis of the male rat to noise stress." $J$ Neuroendocrinol 18(7): 526-533.

Aton, S. J., C. S. Colwell, et al. (2005). "Vasoactive intestinal polypeptide mediates circadian rhythmicity and synchrony in mammalian clock neurons." Nat Neurosci 8(4): 476-483.

Azmi, S., H. Sun, et al. (2003). "mSharp-1/DEC2, a basic helix-loop-helix protein functions as a transcriptional repressor of $E$ box activity and Stra13 expression." J Biol Chem 278(22): 20098-20109.

Bae, K., X. Jin, et al. (2001). "Differential functions of mPer1, mPer2, and mPer3 in the SCN circadian clock." Neuron 30(2): 525-536.

Balsalobre, A., F. Damiola, et al. (1998). "A serum shock induces circadian gene expression in mammalian tissue culture cells." Cell 93(6): 929-937.

Barnard, A. R. and P. M. Nolan (2008). "When clocks go bad: neurobehavioural consequences of disrupted circadian timing." PLoS Genet 4(5): e1000040. 
Bell-Pedersen, D., V. M. Cassone, et al. (2005). "Circadian rhythms from multiple oscillators: lessons from diverse organisms." Nat Rev Genet 6(7): 544-556.

Berson, D. M. (2007). "Phototransduction in ganglion-cell photoreceptors." Pflugers Arch 454(5): 849-855.

Bray, M. S. and M. E. Young (2007). "Circadian rhythms in the development of obesity: potential role for the circadian clock within the adipocyte." Obes Rev 8(2): 169-181.

Buijs, R. M. and A. Kalsbeek (2001). "Hypothalamic integration of central and peripheral clocks." Nat Rev Neurosci 2(7): 521-526.

Bunger, M. K., L. D. Wilsbacher, et al. (2000). "Mop3 is an essential component of the master circadian pacemaker in mammals." Cell 103(7): 1009-1017.

Busino, L., F. Bassermann, et al. (2007). "SCFFbxl3 controls the oscillation of the circadian clock by directing the degradation of cryptochrome proteins." Science 316(5826): 900-904.

Butler, M. P., S. Honma, et al. (2004). "Dec1 and Dec2 expression is disrupted in the suprachiasmatic nuclei of Clock mutant mice." J Biol Rhythms 19(2): 126-134.

Cajochen, C., K. Krauchi, et al. (2003). "Role of melatonin in the regulation of human circadian rhythms and sleep." J Neuroendocrinol 15(4): 432-437.

Cermakian, N., L. Monaco, et al. (2001). "Altered behavioral rhythms and clock gene expression in mice with a targeted mutation in the Period1 gene." EMBO J 20(15): 3967-3974.

Chen, S. T., K. B. Choo, et al. (2005). "Deregulated expression of the PER1, PER2 and PER3 genes in breast cancers." Carcinogenesis 26(7): 1241-1246.

Cheng, M. Y., C. M. Bullock, et al. (2002). "Prokineticin 2 transmits the behavioural circadian rhythm of the suprachiasmatic nucleus." Nature 417(6887): 405-410.

Dardente, H., J. M. Fustin, et al. (2009). "Transcriptional feedback loops in the ovine circadian clock." Comp Biochem Physiol A Mol Integr Physiol 153(4): 391-398.

Debruyne, J. P., E. Noton, et al. (2006). "A clock shock: mouse CLOCK is not required for circadian oscillator function." Neuron 50(3): 465-477.

DeBruyne, J. P., D. R. Weaver, et al. (2007). "CLOCK and NPAS2 have overlapping roles in the suprachiasmatic circadian clock." Nat Neurosci 10(5): 543-545.

Deshmukh, V. D. (2006). "Retino-hypothalamic-pineal hypothesis in the pathophysiology of primary headaches." Med Hypotheses 66(6): 1146-1151.

Dibner, C., U. Schibler, et al. (2010). "The mammalian circadian timing system: organization and coordination of central and peripheral clocks." Annu Rev Physiol 72: 517-549.

Dioum, E. M., J. Rutter, et al. (2002). "NPAS2: a gas-responsive transcription factor." Science 298(5602): 2385-2387.

Ebling, F. J. (1996). "The role of glutamate in the photic regulation of the suprachiasmatic nucleus." Prog Neurobiol 50(2-3): 109-132.

Eide, E. J., E. L. Vielhaber, et al. (2002). "The circadian regulatory proteins BMAL1 and cryptochromes are substrates of casein kinase lepsilon." J Biol Chem 277(19): 17248-17254.

Feillet, C. A., U. Albrecht, et al. (2006). "'Feeding time" for the brain: a matter of clocks." J Physiol Paris 100(5-6): 252-260.

Freedman, M. S., R. J. Lucas, et al. (1999). "Regulation of mammalian circadian behavior by non-rod, non-cone, ocular photoreceptors." Science 284(5413): 502-504.

Fu, L., H. Pelicano, et al. (2002). "The circadian gene Period2 plays an important role in tumor suppression and DNA damage response in vivo." Cell 111(1): 41-50.

Fujimoto, K., M. Shen, et al. (2001). "Molecular cloning and characterization of DEC2, a new member of basic helix-loop-helix proteins." Biochem Biophys Res Commun 280(1): 164-171. 
Gannon, R. L. and M. A. Rea (1994). "In situ hybridization of antisense mRNA oligonucleotides for AMPA, NMDA and metabotropic glutamate receptor subtypes in the rat suprachiasmatic nucleus at different phases of the circadian cycle." Brain Res Mol Brain Res 23(4): 338-344.

Gau, D., T. Lemberger, et al. (2002). "Phosphorylation of CREB Ser142 regulates lightinduced phase shifts of the circadian clock." Neuron 34(2): 245-253.

Gekakis, N., D. Staknis, et al. (1998). "Role of the CLOCK protein in the mammalian circadian mechanism." Science 280(5369): 1564-1569.

Gillette, M. U. and S. A. Tischkau (1999). "Suprachiasmatic nucleus: the brain's circadian clock." Recent Prog Horm Res 54: 33-58; discussion 58-39.

Ginty, D. D., J. M. Kornhauser, et al. (1993). "Regulation of CREB phosphorylation in the suprachiasmatic nucleus by light and a circadian clock." Science 260(5105): 238241.

Godinho, S. I., E. S. Maywood, et al. (2007). "The after-hours mutant reveals a role for Fbxl3 in determining mammalian circadian period." Science 316(5826): 897-900.

Gold, P. W., W. C. Drevets, et al. (2002). "New insights into the role of cortisol and the glucocorticoid receptor in severe depression." Biol Psychiatry 52(5): 381-385.

Golombek, D. A. and R. E. Rosenstein (2010). "Physiology of circadian entrainment." Physiol Rev 90(3): 1063-1102.

Grechez-Cassiau, A., S. Panda, et al. (2004). "The transcriptional repressor STRA13 regulates a subset of peripheral circadian outputs." J Biol Chem 279(2): 11411150.

Green, C. B., J. S. Takahashi, et al. (2008). "The meter of metabolism." Cell 134(5): 728742.

Green, D. J. and R. Gillette (1982). "Circadian rhythm of firing rate recorded from single cells in the rat suprachiasmatic brain slice." Brain Res 245(1): 198-200.

Griffin, E. A., Jr., D. Staknis, et al. (1999). "Light-independent role of CRY1 and CRY2 in the mammalian circadian clock." Science 286(5440): 768-771.

Guillaumond, F., H. Dardente, et al. (2005). "Differential control of Bmal1 circadian transcription by REV-ERB and ROR nuclear receptors." J Biol Rhythms 20(5): 391 403.

Hamaguchi, H., K. Fujimoto, et al. (2004). "Expression of the gene for Dec2, a basic helixloop-helix transcription factor, is regulated by a molecular clock system." Biochem J 382(Pt 1): 43-50.

Hamilton, E. E. and S. A. Kay (2008). "SnapShot: circadian clock proteins." Cell 135(2): 368-368 e361.

Hannibal, J. (2002). "Neurotransmitters of the retino-hypothalamic tract." Cell Tissue Res 309(1): 73-88.

Hannibal, J. (2006). "Roles of PACAP-containing retinal ganglion cells in circadian timing." Int Rev Cytol 251: 1-39.

Hannibal, J., J. M. Ding, et al. (1997). "Pituitary adenylate cyclase-activating peptide (PACAP) in the retinohypothalamic tract: a potential daytime regulator of the biological clock." J Neurosci 17(7): 2637-2644.

Harrington, M. E., D. M. Nance, et al. (1985). "Neuropeptide Y immunoreactivity in the hamster geniculo-suprachiasmatic tract." Brain Res Bull 15(5): 465-472.

Hastings, M., J. S. O'Neill, et al. (2007). "Circadian clocks: regulators of endocrine and metabolic rhythms." J Endocrinol 195(2): 187-198.

Hastings, M. H., G. E. Duffield, et al. (1997). "Non-photic signalling in the suprachiasmatic nucleus." Biol Cell 89(8): 495-503.

Hastings, M. H., G. E. Duffield, et al. (1998). "Entrainment of the circadian system of mammals by nonphotic cues." Chronobiol Int 15(5): 425-445. 
Hattar, S., R. J. Lucas, et al. (2003). "Melanopsin and rod-cone photoreceptive systems account for all major accessory visual functions in mice." Nature 424(6944): 76-81.

Hening, W., R. Allen, et al. (1999). "The treatment of restless legs syndrome and periodic limb movement disorder. An American Academy of Sleep Medicine Review." Sleep 22(7): 970-999.

Hermes, M. L., E. M. Coderre, et al. (1996). "GABA and glutamate mediate rapid neurotransmission from suprachiasmatic nucleus to hypothalamic paraventricular nucleus in rat." J Physiol 496 ( Pt 3): 749-757.

Hirota, T. and Y. Fukada (2004). "Resetting mechanism of central and peripheral circadian clocks in mammals." Zoolog Sci 21(4): 359-368.

Hogenesch, J. B., Y. Z. Gu, et al. (1998). "The basic-helix-loop-helix-PAS orphan MOP3 forms transcriptionally active complexes with circadian and hypoxia factors." Proc Natl Acad Sci U S A 95(10): 5474-5479.

Honma, S., T. Kawamoto, et al. (2002). "Dec1 and Dec2 are regulators of the mammalian molecular clock." Nature 419(6909): 841-844.

Hughes, A. T., C. Guilding, et al. (2008). "Live imaging of altered period1 expression in the suprachiasmatic nuclei of Vipr2-/- mice." J Neurochem 106(4): 1646-1657.

Inouye, S. T. and H. Kawamura (1979). "Persistence of circadian rhythmicity in a mammalian hypothalamic "island" containing the suprachiasmatic nucleus." Proc Natl Acad Sci U S A 76(11): 5962-5966.

Jacob, N., P. Vuillez, et al. (1999). "Does the intergeniculate leaflet play a role in the integration of the photoperiod by the suprachiasmatic nucleus?" Brain Res 828(12): 83-90.

Jakubcakova, V., H. Oster, et al. (2007). "Light entrainment of the mammalian circadian clock by a PRKCA-dependent posttranslational mechanism." Neuron 54(5): 831843.

Jones, C. R., S. S. Campbell, et al. (1999). "Familial advanced sleep-phase syndrome: A short-period circadian rhythm variant in humans." Nat Med 5(9): 1062-1065.

Jud, C., I. Schmutz, et al. (2005). "A guideline for analyzing circadian wheel-running behavior in rodents under different lighting conditions." Biol Proced Online 7: 101116.

Juhl, F., J. Hannibal, et al. (2007). "Photic induction of c-Fos in enkephalin neurons of the rat intergeniculate leaflet innervated by retinal PACAP fibres." Cell Tissue Res 329(3): 491-502.

Kaasik, K. and C. C. Lee (2004). "Reciprocal regulation of haem biosynthesis and the circadian clock in mammals." Nature 430(6998): 467-471.

Kadener, S., D. Stoleru, et al. (2007). "Clockwork Orange is a transcriptional repressor and a new Drosophila circadian pacemaker component." Genes Dev 21(13): 16751686.

Kalsbeek, A. and R. M. Buijs (1992). "Peptidergic transmitters of the suprachiasmatic nuclei and the control of circadian rhythmicity." Prog Brain Res 92: 321-333.

Kalsbeek, A., R. M. Buijs, et al. (1992). "Vasopressin-containing neurons of the suprachiasmatic nuclei inhibit corticosterone release." Brain Res 580(1-2): 62-67.

Kalsbeek, A., F. Kreier, et al. (2007). "Minireview: Circadian control of metabolism by the suprachiasmatic nuclei." Endocrinology 148(12): 5635-5639.

Kalsbeek, A., I. F. Palm, et al. (2006). "SCN outputs and the hypothalamic balance of life." J Biol Rhythms 21(6): 458-469.

Kalsbeek, A., M. Ruiter, et al. (2003). "The diurnal modulation of hormonal responses in the rat varies with different stimuli." J Neuroendocrinol 15(12): 1144-1155. 
Kawamoto, T., M. Noshiro, et al. (2004). "A novel autofeedback loop of Dec1 transcription involved in circadian rhythm regulation." Biochem Biophys Res Commun 313(1): 117-124.

Keller, J., B. Flores, et al. (2006). "Cortisol circadian rhythm alterations in psychotic major depression." Biol Psychiatry 60(3): 275-281.

Khalsa, S. B., M. E. Jewett, et al. (2003). "A phase response curve to single bright light pulses in human subjects." J Physiol 549(Pt 3): 945-952.

Kiessling, S., G. Eichele, et al. (2010). "Adrenal glucocorticoids have a key role in circadian resynchronization in a mouse model of jet lag." J Clin Invest 120(7): 2600-2609.

King, D. P., Y. Zhao, et al. (1997). "Positional cloning of the mouse circadian clock gene." Cell 89(4): 641-653.

Kita, Y., M. Shiozawa, et al. (2002). "Implications of circadian gene expression in kidney, liver and the effects of fasting on pharmacogenomic studies." Pharmacogenetics 12(1): 55-65.

Klein, D. C. and R. Y. Moore (1979). "Pineal N-acetyltransferase and hydroxyindole-Omethyltransferase: control by the retinohypothalamic tract and the suprachiasmatic nucleus." Brain Res 174(2): 245-262.

Konopka, R. J. and S. Benzer (1971). "Clock mutants of Drosophila melanogaster." Proc Natl Acad Sci U S A 68(9): 2112-2116.

Kovac, J., J. Husse, et al. (2009). "A time to fast, a time to feast: the crosstalk between metabolism and the circadian clock." Mol Cells 28(2): 75-80.

Kramer, A., F. C. Yang, et al. (2001). "Regulation of daily locomotor activity and sleep by hypothalamic EGF receptor signaling." Science 294(5551): 2511-2515.

Kraus, T., A. Schuld, et al. (1999). "Periodic leg movements in sleep and restless legs syndrome probably caused by olanzapine." J Clin Psychopharmacol 19(5): 478479.

Kume, K., M. J. Zylka, et al. (1999). "mCRY1 and mCRY2 are essential components of the negative limb of the circadian clock feedback loop." Cell 98(2): 193-205.

Kunz, D. and F. Bes (2001). "Exogenous melatonin in periodic limb movement disorder: an open clinical trial and a hypothesis." Sleep 24(2): 183-187.

Lee, C., J. P. Etchegaray, et al. (2001). "Posttranslational mechanisms regulate the mammalian circadian clock." Cell 107(7): 855-867.

Lee, C. C. (2006). "Tumor suppression by the mammalian Period genes." Cancer Causes Control 17(4): 525-530.

Levi, F. A., C. Canon, et al. (1988). "Circadian rhythms in circulating T lymphocyte subtypes and plasma testosterone, total and free cortisol in five healthy men." Clin Exp Immunol 71(2): 329-335.

Lewy, A. J., H. A. Kern, et al. (1982). "Bright artificial light treatment of a manic-depressive patient with a seasonal mood cycle." Am J Psychiatry 139(11): 1496-1498.

Lewy, A. J. and R. L. Sack (1988). "The phase-shift hypothesis of seasonal affective disorder." Am J Psychiatry 145(8): 1041-1043.

$\mathrm{Li}$, X., N. Sankrithi, et al. (2002). "Transforming growth factor-alpha is expressed in astrocytes of the suprachiasmatic nucleus in hamster: role of glial cells in circadian clocks." Neuroreport 13(16): 2143-2147.

$\mathrm{Li}, \mathrm{Y}$., X. Song, et al. (2004). "DNA binding, but not interaction with Bmal1, is responsible for DEC1-mediated transcription regulation of the circadian gene mPer1." Biochem J 382(Pt 3): 895-904.

$\mathrm{Li}, \mathrm{Y}$., M. Xie, et al. (2003). "DEC1 negatively regulates the expression of DEC2 through binding to the E-box in the proximal promoter." J Biol Chem 278(19): 16899-16907. 
Lim, C., B. Y. Chung, et al. (2007). "Clockwork orange encodes a transcriptional repressor important for circadian-clock amplitude in Drosophila." Curr Biol 17(12): 1082-1089.

Linkowski, P., J. Mendlewicz, et al. (1985). "The 24-hour profile of adrenocorticotropin and cortisol in major depressive illness." J Clin Endocrinol Metab 61(3): 429-438.

Liu, A. C., D. K. Welsh, et al. (2007). "Intercellular coupling confers robustness against mutations in the SCN circadian clock network." Cell 129(3): 605-616.

Liu, C. and S. M. Reppert (2000). "GABA synchronizes clock cells within the suprachiasmatic circadian clock." Neuron 25(1): 123-128.

Liu, Y., F. Sato, et al. (2010). "Anti-apoptotic effect of the basic helix-loop-helix (bHLH) transcription factor DEC2 in human breast cancer cells." Genes Cells 15(4): 315325.

Looby, P. and A. S. Loudon (2005). "Gene duplication and complex circadian clocks in mammals." Trends Genet 21(1): 46-53.

Lucas, R. J., M. S. Freedman, et al. (2001). "Identifying the photoreceptive inputs to the mammalian circadian system using transgenic and retinally degenerate mice." Behav Brain Res 125(1-2): 97-102.

Marchant, E. G. and R. E. Mistlberger (1995). "Morphine phase-shifts circadian rhythms in mice: role of behavioural activation." Neuroreport 7(1): 209-212.

Matsumoto, A., M. Ukai-Tadenuma, et al. (2007). "A functional genomics strategy reveals clockwork orange as a transcriptional regulator in the Drosophila circadian clock." Genes Dev 21(13): 1687-1700.

Maywood, E. S., J. S. O'Neill, et al. (2007). "Minireview: The circadian clockwork of the suprachiasmatic nuclei--analysis of a cellular oscillator that drives endocrine rhythms." Endocrinology 148(12): 5624-5634.

Maywood, E. S., A. B. Reddy, et al. (2006). "Synchronization and maintenance of timekeeping in suprachiasmatic circadian clock cells by neuropeptidergic signaling." Curr Biol 16(6): 599-605.

McCarthy, J. J., J. L. Andrews, et al. (2007). "Identification of the circadian transcriptome in adult mouse skeletal muscle." Physiol Genomics 31(1): 86-95.

Mendoza, J. (2007). "Circadian clocks: setting time by food." J Neuroendocrinol 19(2): 127-137.

Michaud, M., M. Dumont, et al. (2004). "Circadian rhythm of restless legs syndrome: relationship with biological markers." Ann Neurol 55(3): 372-380.

Mick, G., R. Yoshimura, et al. (1995). "The messenger RNAs encoding metabotropic glutamate receptor subtypes are expressed in different neuronal subpopulations of the rat suprachiasmatic nucleus." Neuroscience 66(1): 161-173.

Mistlberger, R. E. (1994). "Circadian food-anticipatory activity: formal models and physiological mechanisms." Neurosci Biobehav Rev 18(2): 171-195.

Miyake, S., Y. Sumi, et al. (2000). "Phase-dependent responses of Per1 and Per2 genes to a light-stimulus in the suprachiasmatic nucleus of the rat." Neurosci Lett 294(1): 41-44.

Mizoro, Y., Y. Yamaguchi, et al. (2010). "Activation of AMPA receptors in the suprachiasmatic nucleus phase-shifts the mouse circadian clock in vivo and in vitro." PLoS One 5(6): e10951.

Moore, R. Y. and J. P. Card (1994). "Intergeniculate leaflet: an anatomically and functionally distinct subdivision of the lateral geniculate complex." J Comp Neurol 344(3): 403-430.

Moore, R. Y. and V. B. Eichler (1972). "Loss of a circadian adrenal corticosterone rhythm following suprachiasmatic lesions in the rat." Brain Res 42(1): 201-206.

Moore, R. Y. and N. J. Lenn (1972). "A retinohypothalamic projection in the rat." J Comp Neurol 146(1): 1-14. 
Moore, R. Y., J. C. Speh, et al. (2002). "Suprachiasmatic nucleus organization." Cell Tissue Res 309(1): 89-98.

Mrosovsky, N. (1988). "Phase response curves for social entrainment." J Comp Physiol A 162(1): 35-46.

Mrosovsky, N. (1996). "Locomotor activity and non-photic influences on circadian clocks." Biol Rev Camb Philos Soc 71(3): 343-372.

Mrosovsky, N., S. G. Reebs, et al. (1989). "Behavioural entrainment of circadian rhythms." Experientia 45(8): 696-702.

Nader, N., G. P. Chrousos, et al. (2010). "Interactions of the circadian CLOCK system and the HPA axis." Trends Endocrinol Metab 21(5): 277-286.

Nagoshi, E., C. Saini, et al. (2004). "Circadian gene expression in individual fibroblasts: cell-autonomous and self-sustained oscillators pass time to daughter cells." Cell 119(5): 693-705.

Nakahata, Y., S. Sahar, et al. (2009). "Circadian control of the NAD+ salvage pathway by CLOCK-SIRT1." Science 324(5927): 654-657.

Nakashima, A., T. Kawamoto, et al. (2008). "DEC1 modulates the circadian phase of clock gene expression." Mol Cell Biol 28(12): 4080-4092.

Noshiro, M., M. Furukawa, et al. (2005). "Tissue-specific disruption of rhythmic expression of Dec1 and Dec2 in clock mutant mice." J Biol Rhythms 20(5): 404-418.

Obrietan, K., S. Impey, et al. (1999). "Circadian regulation of cAMP response elementmediated gene expression in the suprachiasmatic nuclei." J Biol Chem 274(25): 17748-17756.

Obrietan, K., S. Impey, et al. (1998). "Light and circadian rhythmicity regulate MAP kinase activation in the suprachiasmatic nuclei." Nat Neurosci 1(8): 693-700.

Okamura, H., S. Miyake, et al. (1999). "Photic induction of mPer1 and mPer2 in crydeficient mice lacking a biological clock." Science 286(5449): 2531-2534.

Oster, H. (2006). "The genetic basis of circadian behavior." Genes Brain Behav 5 Suppl 2: 73-79.

Oster, H., S. Baeriswyl, et al. (2003). "Loss of circadian rhythmicity in aging mPer1-/mCry2-/- mutant mice." Genes Dev 17(11): 1366-1379.

Oster, H., A. Yasui, et al. (2002). "Disruption of mCry2 restores circadian rhythmicity in mPer2 mutant mice." Genes Dev 16(20): 2633-2638.

Ouyang, Y., C. R. Andersson, et al. (1998). "Resonating circadian clocks enhance fitness in cyanobacteria." Proc Natl Acad Sci U S A 95(15): 8660-8664.

Panda, S., M. P. Antoch, et al. (2002). "Coordinated transcription of key pathways in the mouse by the circadian clock." Cell 109(3): 307-320.

Panda, S. and J. B. Hogenesch (2004). "It's all in the timing: many clocks, many outputs." J Biol Rhythms 19(5): 374-387.

Papadimitriou, A. and K. N. Priftis (2009). "Regulation of the hypothalamic-pituitaryadrenal axis." Neuroimmunomodulation 16(5): 265-271.

Paparrigopoulos, T. (2002). "Melatonin response to atenolol administration in depression: indication of beta-adrenoceptor dysfunction in a subtype of depression." Acta Psychiatr Scand 106(6): 440-445.

Persaud, R. (2000). "Nocturnal sweating and temperature in depression'." Acta Psychiatr Scand 101(3): 251.

Pittendrigh, C. S. (1993). "Temporal organization: reflections of a Darwinian clockwatcher." Annu Rev Physiol 55: 16-54.

Pittendrigh, S. D. a. C. S. (1976). "A Functional Analysis of Circadian Pacemakers in Nocturnal Rodents II. The Variability of Phase Response Curves." J. comp. Physiol(106): 253-266. 
Preitner, N., F. Damiola, et al. (2002). "The orphan nuclear receptor REV-ERBalpha controls circadian transcription within the positive limb of the mammalian circadian oscillator." Cell 110(2): 251-260.

Provencio, I., M. D. Rollag, et al. (2002). "Photoreceptive net in the mammalian retina. This mesh of cells may explain how some blind mice can still tell day from night." Nature 415(6871): 493.

Ralph, M. R., R. G. Foster, et al. (1990). "Transplanted suprachiasmatic nucleus determines circadian period." Science 247(4945): 975-978.

Ramsey, K. M., J. Yoshino, et al. (2009). "Circadian clock feedback cycle through NAMPT-mediated NAD+ biosynthesis." Science 324(5927): 651-654.

Reddy, A. B., N. A. Karp, et al. (2006). "Circadian orchestration of the hepatic proteome." Curr Biol 16(11): 1107-1115.

Reebs, S. G. and N. Mrosovsky (1989). "Effects of induced wheel running on the circadian activity rhythms of Syrian hamsters: entrainment and phase response curve." J Biol Rhythms 4(1): 39-48.

Reick, M., J. A. Garcia, et al. (2001). "NPAS2: an analog of clock operative in the mammalian forebrain." Science 293(5529): 506-509.

Reinberg, A. and M. H. Smolensky (1982). "Circadian changes of drug disposition in man." Clin Pharmacokinet 7(5): 401-420.

Reppert, S. M. and D. R. Weaver (2002). "Coordination of circadian timing in mammals." Nature 418(6901): 935-941.

Richier, B., C. Michard-Vanhee, et al. (2008). "The clockwork orange Drosophila protein functions as both an activator and a repressor of clock gene expression." J Biol Rhythms 23(2): 103-116.

Richier, S., M. Rodriguez-Lanetty, et al. (2008). "Response of the symbiotic cnidarian Anthopleura elegantissima transcriptome to temperature and UV increase." Comp Biochem Physiol Part D Genomics Proteomics 3(4): 283-289.

Rosbash, M. (2009). "The implications of multiple circadian clock origins." PLoS Biol 7(3): e62.

Rosenthal, N. E., D. A. Sack, et al. (1984). "Seasonal affective disorder. A description of the syndrome and preliminary findings with light therapy." Arch Gen Psychiatry 41(1): 72-80.

Rossner, M. J., H. Oster, et al. (2008). "Disturbed clockwork resetting in Sharp-1 and Sharp-2 single and double mutant mice." PLoS ONE 3(7): e2762.

Roybal, K., D. Theobold, et al. (2007). "Mania-like behavior induced by disruption of CLOCK." Proc Natl Acad Sci U S A 104(15): 6406-6411.

Rutter, J., M. Reick, et al. (2001). "Regulation of clock and NPAS2 DNA binding by the redox state of NAD cofactors." Science 293(5529): 510-514.

Sage, D., D. Maurel, et al. (2001). "Involvement of the suprachiasmatic nucleus in diurnal ACTH and corticosterone responsiveness to stress." Am J Physiol Endocrinol Metab 280(2): E260-269.

Saper, C. B., G. Cano, et al. (2005). "Homeostatic, circadian, and emotional regulation of sleep." J Comp Neurol 493(1): 92-98.

Sato, F., T. Kawamoto, et al. (2004). "Functional analysis of the basic helix-loop-helix transcription factor DEC1 in circadian regulation. Interaction with BMAL1." Eur J Biochem 271(22): 4409-4419.

Sato, T. K., S. Panda, et al. (2004). "A functional genomics strategy reveals Rora as a component of the mammalian circadian clock." Neuron 43(4): 527-537.

Sato, T. K., R. G. Yamada, et al. (2006). "Feedback repression is required for mammalian circadian clock function." Nat Genet 38(3): 312-319. 
Schernhammer, E. S., F. Berrino, et al. (2008). "Urinary 6-sulfatoxymelatonin levels and risk of breast cancer in postmenopausal women." J Natl Cancer Inst 100(12): 898905.

Schernhammer, E. S., F. Laden, et al. (2003). "Night-shift work and risk of colorectal cancer in the nurses' health study." J Natl Cancer Inst 95(11): 825-828.

Shearman, L. P., X. Jin, et al. (2000). "Targeted disruption of the mPer3 gene: subtle effects on circadian clock function." Mol Cell Biol 20(17): 6269-6275.

Shearman, L. P., S. Sriram, et al. (2000). "Interacting molecular loops in the mammalian circadian clock." Science 288(5468): 1013-1019.

Shearman, L. P., M. J. Zylka, et al. (1997). "Two period homologs: circadian expression and photic regulation in the suprachiasmatic nuclei." Neuron 19(6): 1261-1269.

Shen, M., T. Kawamoto, et al. (1997). "Molecular characterization of the novel basic helixloop-helix protein DEC1 expressed in differentiated human embryo chondrocytes." Biochem Biophys Res Commun 236(2): 294-298.

Shibata, S., Y. Oomura, et al. (1982). "Circadian rhythmic changes of neuronal activity in the suprachiasmatic nucleus of the rat hypothalamic slice." Brain Res 247(1): 154158.

Shigeyoshi, Y., K. Taguchi, et al. (1997). "Light-induced resetting of a mammalian circadian clock is associated with rapid induction of the mPer1 transcript." Cell 91(7): 1043-1053.

Siepka, S. M., S. H. Yoo, et al. (2007). "Circadian mutant Overtime reveals F-box protein FBXL3 regulation of cryptochrome and period gene expression." Cell 129(5): 10111023.

Silver, R., J. LeSauter, et al. (1996). "A diffusible coupling signal from the transplanted suprachiasmatic nucleus controlling circadian locomotor rhythms." Nature 382(6594): 810-813.

So, A. Y., T. U. Bernal, et al. (2009). "Glucocorticoid regulation of the circadian clock modulates glucose homeostasis." Proc Natl Acad Sci U S A 106(41): 17582-17587.

Spoelstra, K., U. Albrecht, et al. (2004). "Phase responses to light pulses in mice lacking functional per or cry genes." J Biol Rhythms 19(6): 518-529.

Srinivasan, V., J. Singh, et al. "Jet lag, circadian rhythm sleep disturbances, and depression: the role of melatonin and its analogs." Adv Ther 27(11): 796-813.

Stephan, F. K. (2002). "The "other" circadian system: food as a Zeitgeber." J Biol Rhythms 17(4): 284-292.

Stephan, F. K. and I. Zucker (1972). "Circadian rhythms in drinking behavior and locomotor activity of rats are eliminated by hypothalamic lesions." Proc Natl Acad Sci U S A 69(6): 1583-1586.

Storch, K. F., O. Lipan, et al. (2002). "Extensive and divergent circadian gene expression in liver and heart." Nature 417(6884): 78-83.

Sun, H., B. Lu, et al. (2001). "Defective T cell activation and autoimmune disorder in Stra13-deficient mice." Nat Immunol 2(11): 1040-1047.

Toh, K. L., C. R. Jones, et al. (2001). "An hPer2 phosphorylation site mutation in familial advanced sleep phase syndrome." Science 291(5506): 1040-1043.

Travis, R. C., D. S. Allen, et al. (2004). "Melatonin and breast cancer: a prospective study." J Natl Cancer Inst 96(6): 475-482.

Tribl, G. G., F. Waldhauser, et al. (2003). "Urinary 6-hydroxy-melatonin-sulfate excretion and circadian rhythm in patients with restless legs syndrome." J Pineal Res 35(4): 295-296.

Triqueneaux, G., S. Thenot, et al. (2004). "The orphan receptor Rev-erbalpha gene is a target of the circadian clock pacemaker." J Mol Endocrinol 33(3): 585-608. 
Turek, F. W., C. Joshu, et al. (2005). "Obesity and metabolic syndrome in circadian Clock mutant mice." Science 308(5724): 1043-1045.

Ueda, H. R., W. Chen, et al. (2002). "A transcription factor response element for gene expression during circadian night." Nature 418(6897): 534-539.

van der Horst, G. T., M. Muijtjens, et al. (1999). "Mammalian Cry1 and Cry2 are essential for maintenance of circadian rhythms." Nature 398(6728): 627-630.

Van Reeth, O. and F. W. Turek (1989). "Stimulated activity mediates phase shifts in the hamster circadian clock induced by dark pulses or benzodiazepines." Nature 339(6219): 49-51.

Vitaterna, M. H., D. P. King, et al. (1994). "Mutagenesis and mapping of a mouse gene, Clock, essential for circadian behavior." Science 264(5159): 719-725.

Vitaterna, M. H., C. P. Selby, et al. (1999). "Differential regulation of mammalian period genes and circadian rhythmicity by cryptochromes 1 and 2." Proc Natl Acad Sci U S A 96(21): 12114-12119.

Vosko, A. M., A. Schroeder, et al. (2007). "Vasoactive intestinal peptide and the mammalian circadian system." Gen Comp Endocrinol 152(2-3): 165-175.

Walters, A. S. (1995). "Toward a better definition of the restless legs syndrome. The International Restless Legs Syndrome Study Group." Mov Disord 10(5): 634-642.

Waterhouse, J., T. Reilly, et al. (2007). "Jet lag: trends and coping strategies." Lancet 369(9567): 1117-1129.

Welsh, D. K., D. E. Logothetis, et al. (1995). "Individual neurons dissociated from rat suprachiasmatic nucleus express independently phased circadian firing rhythms." Neuron 14(4): 697-706.

Welsh, D. K., S. H. Yoo, et al. (2004). "Bioluminescence imaging of individual fibroblasts reveals persistent, independently phased circadian rhythms of clock gene expression." Curr Biol 14(24): 2289-2295.

Wetter, T. C., V. Collado-Seidel, et al. (2002). "Endocrine rhythms in patients with restless legs syndrome." J Neurol 249(2): 146-151.

Wirz-Justice, A., C. Bucheli, et al. (1986). "Light treatment of seasonal affective disorder in Switzerland." Acta Psychiatr Scand 74(2): 193-204.

Yamada, K. and K. Miyamoto (2005). "Basic helix-loop-helix transcription factors, BHLHB2 and BHLHB3; their gene expressions are regulated by multiple extracellular stimuli." Front Biosci 10: 3151-3171.

Yamaguchi, S., H. Isejima, et al. (2003). "Synchronization of cellular clocks in the suprachiasmatic nucleus." Science 302(5649): 1408-1412.

Yamazaki, S., R. Numano, et al. (2000). "Resetting central and peripheral circadian oscillators in transgenic rats." Science 288(5466): 682-685.

Yamazaki, S., T. Yoshikawa, et al. (2009). "Ontogeny of circadian organization in the rat." J Biol Rhythms 24(1): 55-63.

Yan, L. and R. Silver (2002). "Differential induction and localization of mPer1 and mPer2 during advancing and delaying phase shifts." Eur J Neurosci 16(8): 1531-1540.

Yan, L. and R. Silver (2004). "Resetting the brain clock: time course and localization of mPER1 and mPER2 protein expression in suprachiasmatic nuclei during phase shifts." Eur J Neurosci 19(4): 1105-1109.

Yan, L., S. Takekida, et al. (1999). "Per1 and Per2 gene expression in the rat suprachiasmatic nucleus: circadian profile and the compartment-specific response to light." Neuroscience 94(1): 141-150.

Yin, L., N. Wu, et al. (2007). "Rev-erbalpha, a heme sensor that coordinates metabolic and circadian pathways." Science 318(5857): 1786-1789.

Yokota, S., M. Yamamoto, et al. (2001). "Involvement of calcium-calmodulin protein kinase but not mitogen-activated protein kinase in light-induced phase delays and 
Per gene expression in the suprachiasmatic nucleus of the hamster." J Neurochem 77(2): 618-627.

Yoo, S. H., S. Yamazaki, et al. (2004). "PERIOD2::LUCIFERASE real-time reporting of circadian dynamics reveals persistent circadian oscillations in mouse peripheral tissues." Proc Natl Acad Sci U S A 101(15): 5339-5346.

Zhang, E. E. and S. A. Kay (2010). "Clocks not winding down: unravelling circadian networks." Nat Rev Mol Cell Biol 11(11): 764-776.

Zhang, E. E., A. C. Liu, et al. (2009). "A genome-wide RNAi screen for modifiers of the circadian clock in human cells." Cell 139(1): 199-210.

Zheng, B., U. Albrecht, et al. (2001). "Nonredundant roles of the mPer1 and mPer2 genes in the mammalian circadian clock." Cell 105(5): 683-694.

Zheng, B., D. W. Larkin, et al. (1999). "The mPer2 gene encodes a functional component of the mammalian circadian clock." Nature 400(6740): 169-173.

Zvonic, S., A. A. Ptitsyn, et al. (2006). "Characterization of peripheral circadian clocks in adipose tissues." Diabetes 55(4): 962-970. 


\section{Acknowledgements}

First of all I would like to thank Dr. Henrik Oster for his supervision, patience and all the support and guidance he gave me throughout the last years.

Thanks also to the members of my thesis committee, Prof. Julia Fischer and Prof. Walter Paulus who support my thesis. I would like to acknowledge all the members of the PhD program.

Moreover, I would like to thank Dr. Moritz Rossner and Ali Shahmoradi from the Max Planck Institute for Experimental Medicine, Göttingen, for their collaboration.

Thanks to all the people at the Max Planck Institute for Biophysical Chemistry, Göttingen, who helped me considerably to finish this work, especially Alexei Leliavski and Johanna Barclay who did an excellent job in revising this thesis. I want to thank Jana Husse and the whole Circadian Rhythm Group: Dominic Landgraf, Anton Shostak, Anthony Tsang, Judit Meyer-Kovac, Christin Helbig and especially Nadine Naujokat. Further, I would like to thank Helena Miletic, Christine van den Bogaart and my animal caretaker Alexandra Driehorst.

Most importantly, I would like thank my family and my friends, especially Jana Illiger, for their support during the last years. Their patience and emotional support brought me a good deal closer to my aims.

Finally, I would like to thank all of my mice.

Brid,

Göttingen, 20.April 2011 


\section{Curriculum Vitae}

Brid Bode

Geboren am 25. September 1980 in Wolfenbüttel, Deutschland

Gartenstraße 3, 37073 Göttingen

E-Mail brid.bode@mpibpc.de

\section{Beruflicher Werdegang}

seit 2007

Wissenschaftliche Mitarbeiterin am Max-Planck-Institut für biophysikalische Chemie, Göttingen, Deutschland, Abtl. Gene und Verhalten, Forschungsgruppe Zirkadiane Rhythmen

Doktorarbeit: ,Genetic interaction of Per- and Dec-genes in the mammalian circadian clock'

seit 2007

Doktorandenprogramm an der Göttinger Graduiertenschule für Neurowissenschaften und Molekulare Biowissenschaften (GGNB): Zentrum für Neurobiologie des Verhaltens (CSN)

2001- 2007

Studium der Biologie an der Georg-August Universität Göttingen, Deutschland; Schwerpunkte Entwicklungsbiologie, Mikrobiologie und Immunologie; Abschluss mit Diplom 05/2007

1993 - $2001 \quad$ Gymnasium im Schloss Wolfenbüttel, Abitur 2001 